\title{
Transport mirages in single-molecule devices
}

\author{
R. Gaudenzi, ${ }^{1}$ M. Misiorny, ${ }^{2,3}$ E. Burzurí, ${ }^{1}$ M. R. Wegewijs, ${ }^{4,5,6}$ and H. S. J. van der Zant ${ }^{1}$ \\ ${ }^{1}$ Kavli Institute of Nanoscience, Delft University of Technology, 2600 GA Delft, The Netherlands \\ ${ }^{2}$ Department of Microtechnology and Nanoscience MC2, Chalmers University of Technology, \\ 41296 Göteborg, Sweden \\ ${ }^{3}$ Faculty of Physics, Adam Mickiewicz University, 61-614 Poznań, Poland \\ ${ }^{4}$ Peter Grünberg Institut, Forschungszentrum Jülich, 52425 Jülich, Germany \\ ${ }^{5}$ JARA-FIT, 52056 Aachen, Germany \\ ${ }^{6}$ Institute for Theory of Statistical Physics, RWTH Aachen, 52056 Aachen, Germany
}

(Received 2 November 2016; accepted 25 January 2017; published online 23 February 2017)

\begin{abstract}
Molecular systems can exhibit a complex, chemically tailorable inner structure which allows for targeting of specific mechanical, electronic, and optical properties. At the single-molecule level, two major complementary ways to explore these properties are molecular quantum-dot structures and scanning probes. This article outlines comprehensive principles of electron-transport spectroscopy relevant to both these approaches and presents a new, high-resolution experiment on a high-spin single-molecule junction exemplifying these principles. Such spectroscopy plays a key role in further advancing our understanding of molecular and atomic systems, in particular, the relaxation of their spin. In this joint experimental and theoretical analysis, particular focus is put on the crossover between the resonant regime [single-electron tunneling] and the off-resonant regime [inelastic electron (co)tunneling spectroscopy (IETS)]. We show that the interplay of these two processes leads to unexpected mirages of resonances not captured by either of the two pictures alone. Although this turns out to be important in a large fraction of the possible regimes of level positions and bias voltages, it has been given little attention in molecular transport studies. Combined with nonequilibrium IETSfour-electron pump-probe excitations - these mirages provide crucial information on the relaxation of spin excitations. Our encompassing physical picture is supported by a master-equation approach that goes beyond weak coupling. The present work encourages the development of a broader connection between the fields of molecular quantum-dot and scanning probe spectroscopy. Published by AIP Publishing. [http://dx.doi.org/10.1063/1.4975767]
\end{abstract}

\section{INTRODUCTION}

Both the fundamental and applied studies on transport phenomena in electronic devices of molecular dimensions have bloomed over the past decade. ${ }^{1-4}$ An interesting aspect of this development is that it has increasingly hybridized the diverse fields of chemistry, nanofabrication, and physics with the primary ambition of accessing properties like high spin and large exchange couplings, vibrational modes, large charging energies and long electronic/nuclear spin coherence times, subtle electronic orbital interplay, self-organisation, ${ }^{5,6}$ and chirality. ${ }^{7,8}$ This is rendered possible by the higher energy scales of the molecular systems - a direct consequence of their sizeand their complex, chemically tailorable, inner structures which have proven to be effective in addressing, for instance, the spin-phonon, ${ }^{9,10}$ Shiba ${ }^{11,12}$ and Kondo physics ${ }^{13}$ quantum interference effects, ${ }^{14}$ and nuclear spin manipulation. ${ }^{15}$

In most of the works, in particular, those concerning molecular spin systems, two complementary approaches have contributed to explore these effects. On the one hand stands off-resonant transport spectroscopy, which is the major tool of choice in the scanning-tunneling microscopy (STM) approach to nanoscale spin systems, ${ }^{16-27}$ depicted in Fig. 1(a). Off-resonant spectroscopy is also dominant in the field of mechanically controlled break junctions (MCBJ) ${ }^{28,29}$ to study vibrations $^{9,30-33}$ and, less often, spin effects. ${ }^{13,34,35}$ On the other hand, resonant transport spectroscopy, originating in the multi-terminal fabrication of quantum dots (QDs, Fig. 1(b)), ${ }^{36}$ is a well-developed tool applied to a broad range of excitations in nanostructures, ${ }^{37-46}$ including spin. ${ }^{47-57}$ The key difference between resonant and off-resonant approaches is the former's reliance on energy-level control independent of the transport bias, i.e., true gating of the molecular levels, ${ }^{58-62}$ which should be distinguished from the capacitive level shift in STM which is caused by the bias. In terms of physical processes, this difference corresponds to resonant spectroscopy relying on real charging of the molecule and off-resonant transport involving only virtual charging.

In this contribution, we discuss a comprehensive picture of transport applicable to a large family of nanoscale objects. This is motivated by the experimental spectrum of a molecular junction depicted in Fig. 1(c). Such a conductance map is so full of detail that it warrants a systematic joint experimental and theoretical study. In particular, we discuss several effects which are often overlooked despite their importance to electron transport spectroscopy and despite existing experimental ${ }^{33,63,64}$ and theoretical works. ${ }^{65-70}$ For instance, it turns out that inelastic/off-resonant transport is not simply equivalent to the statement that "resonant processes play no role." In fact, we show that generally less than $55 \%$ of the parameter regime of applied voltages that nominally qualified as off-resonant is actually described by the widely used inelastic 
a

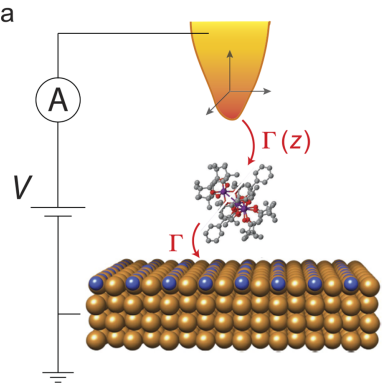

b

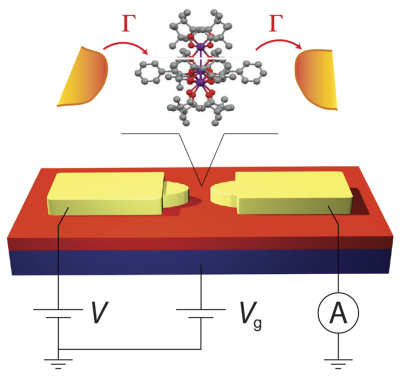

C

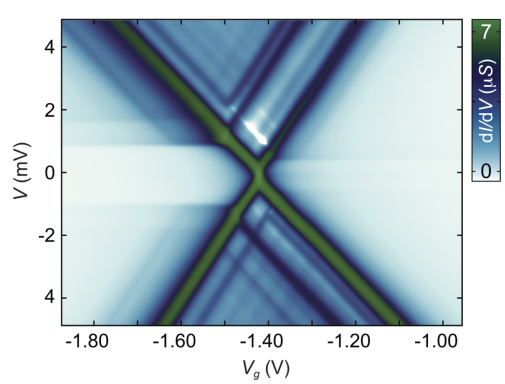

FIG. 1. High-spin single-molecule junctions. (a) Vertical approach: the metallic tip of a scanning tunneling microscope (STM) allows one to scan laterally in real space and acquire transport spectra as a function of the bias $V$ at specific molecular sites. The vertical position $z$ controls the tip-molecule coupling $\Gamma(z)$, while the molecule-substrate coupling is fixed. (b) Planar approach: by embedding a bottom-up synthesized magnetic molecule into a solid-state device, one can control its energy levels through a gate-voltage $V_{\mathrm{g}}$. This scanning in energy space grants access to both regimes of real (redox) charging and virtual charging (scattering) and their nontrivial crossover. (c) Conductance map showing a range of features in the resonant regime (center), off-resonant regimes (far left and right), as well as the crossover regime. These are analyzed in detail in Figs. 11 and 16.

(co)tunneling spectroscopy (COT or IETS) picture. Although in many experiments to date this has not been so apparent, our experimental evidence suggests that this needs consideration. In theoretical considerations, resonant and off-resonant transport regimes are often taken as complementary. Our measurements illustrate how this overlooks an important class of relaxation processes. The breakdown of the COT picture in the off-resonant regime presents, in fact, new opportunities for studying the relaxation of molecular spin-excitations which are of importance for applications. Interestingly, these resonances are qualitative indicators of a device of high quality, e.g., for applications involving spin-pumping. We illustrate experimentally the ambiguities that the sole modeling of off-resonant conductance curves can run into. For instance,

we show that this may lead one to infer quantum states that do not correspond to real excitations, but are simply mirages of lower lying excitations, including their Zeeman splittings. Although elaborated here for a spin system, our conclusions apply generally, for example, to electronic ${ }^{63}$ and vibrational excitations in nano electro-mechanical systems (NEMS). ${ }^{33,64}$

The outline of the paper is as follows: In Sec. II, we review the physical picture of electron tunneling spectroscopy and outline how a given spectrum manifests itself in resonant [Sec. II A] and off-resonant [Sec. II B] transport spectra. In Sec. II C, we discuss how these two spectra continuously transform into each other as the energy levels are varied relative to the bias voltage. With this in hand, we put together a physical picture capturing all discussed effects which will be subsequently applied to describe the experiment in Sec. III.

In Sec. III, we follow the reverse path of experimental transport spectroscopy: We reconstruct the excitation spectrum of a high-spin molecular junction based on the feature-rich transport spectra as a function of bias voltage, magnetic field, and gate voltage. Starting from the off-resonant analysis, we use the boundary conditions imposed by the resonant spectrum to resolve a number of ambiguities in the off-resonant state-assignment. With the full model in hand, we highlight two informative transport features: (i) nonequilibrium COT, i.e., a pump-probe spectroscopy using the electronic analog of Raman transitions and (ii) mirages of single-electron tunneling (SET) resonances that occur well inside the off-resonant regime. We conclude with an outlook in Sec. IV.

Since we aim to bring the insights from various communities together, we summarize in Table I the different but equivalent terminology used. For clarity reasons, we set $k_{\mathrm{B}}=\hbar=e=1$ for the rest of this discussion.

\section{PHYSICAL PICTURES OF TRANSPORT-REAL VS. VIRTUAL CHARGING}

The two prevalent conceptual approaches to transport through molecular electronic devices are characterized by the simple physical distinction, sketched in Fig. 2, between real charging - chemical reduction or oxidation-and virtual charging - electrons scattering between contacts through a molecular bridge. Theoretically, the distinction rests on whether the physical processes appear in the leading or nextto-leading order in the tunnel coupling strength, $\Gamma$, relative to the thermal fluctuation energy $T$. Experimentally, this translates into distinct applied voltages under which these processes turn on. These conditions are the primary spectroscopic

TABLE I. Nomenclature of off-resonant and resonant spectroscopy in different communities.

\begin{tabular}{|c|c|c|c|}
\hline Regime & Section & QD community & STM/MCBJ community \\
\hline Resonant & Sec. II A & $\begin{array}{l}\text { Single-electron tunneling (SET) } \\
\text { Sequential/incoherent tunneling }\end{array}$ & Resonant tunneling \\
\hline Off-resonant & Sec. II B & $\begin{array}{l}\text { (In)elastic co-tunneling (COT) } \\
\text { Coherent tunneling } \\
\text { Schrieffer-Wolff (transformation) }\end{array}$ & $\begin{array}{l}\text { (In)elastic electron tunneling spectroscopy } \\
\text { (EETS/IETS) } \\
\text { Appelbaum (Hamiltonian) }\end{array}$ \\
\hline Crossover & $\begin{array}{l}\text { Sec. II B } 1 \\
\text { Sec. II C }\end{array}$ & $\begin{array}{l}\text { Cotunneling-assisted single-electron } \\
\text { Tunneling (COSET, CAST) }\end{array}$ & Pump-probe (co)tunneling spectroscopy \\
\hline
\end{tabular}




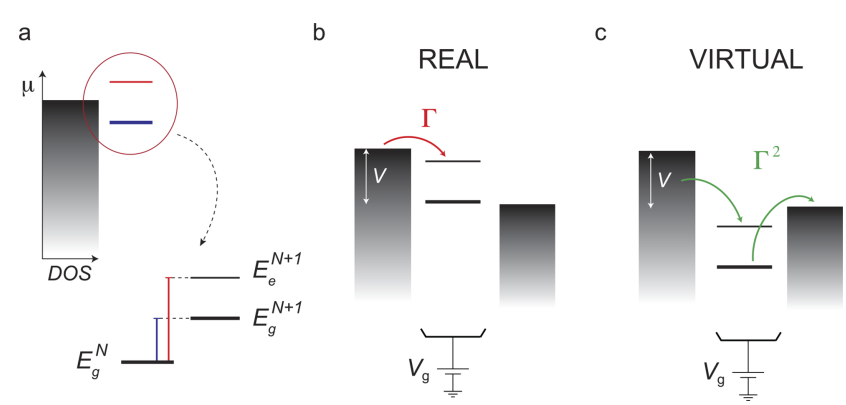

FIG. 2. Real and virtual transport processes. (a) Electrochemical potential $(\boldsymbol{\mu})$ picture (top) and corresponding molecular energy level picture (bottom). Each discrete "level" in the top panel stands for an electrochemical potential of the molecule, i.e., a difference between two energies sketched in the lower panel, $E_{g}^{N+1}-E_{g}^{N}$ (dark blue) and $E_{e}^{N+1}-E_{g}^{N}$ (red). The energies $E_{i}^{N}$ depend on the charge $N$ and further quantum numbers denoted by $i$. Due to the capacitive coupling to a gate electrode, these energy differences can be tuned to be (b) onresonance and (c) off-resonance with the electrode continuum indicated by the gray shaded boxes. (b) Real charging: absorption of an electron, reduces the molecule for real, $(N, g) \rightarrow(N+1, e)$, going from the ground state $g$ for charge $N$ to an excited state $e$ with charge $N+1$. Since this is a one-step process, the rate scales with $\Gamma$, the strength of the tunnel coupling. The applied voltage $V$ equals the difference between the electrochemical potentials of the electrodes. (c) Virtual charging: the scattering of an electron off or through the molecule proceeds via a virtual intermediate state, for example, starting from the ground state $g$ and ending in a final excited state $e,(N+1, g) \rightarrow(N, g) \rightarrow(N+1, e)$. The rate of such a two-step process scales as $\Gamma^{2}$. In this case, charging is considered only virtual, as no redox reaction takes place: although energy and angular momentum are transferred onto the molecule, the electron number remains fixed to $N+1$.

indicators, allowing the distinction between real and virtual transport processes, and take precedence over line shape and lifetime broadening. For reviews on theoretical approaches to molecular transport, see Refs. 3 and 71-73.

\section{A. Resonant transport spectroscopy}

Real charging forms the starting point of what we will call the resonant picture of transport (see Table I for other nomenclature). Its energy resolution is limited by the Heisenberg lifetime set by the tunnel coupling $\mathcal{H}^{T}$,

$$
\left(\mathcal{H}^{\mathrm{T}}\right)^{2} \propto \Gamma,
$$

allowing for sharp transport spectroscopy of weakly coupled systems. This relation has a prominent place in the field of QDs which covers structures such as artificial atoms and artificial molecules with redox spectra ${ }^{36}$ very similar to real atoms ${ }^{74}$ and simple molecules. ${ }^{42,55,75-77}$ Resonant transport also plays a role in STM although its energy resolution is often limited by the strong coupling typical of the asymmetric probe-substrate configuration.

Given sufficient weak coupling/energy resolution, much is gained when the energy-level dependence of these transport spectra, can be mapped out as function of gate-voltage. This dependence allows a detailed model to be extracted involving just a few electronic orbitals, ${ }^{75,78}$ their Coulomb interactions, ${ }^{79}$ and their interaction with the most relevant degrees of freedom (e.g., isotropic ${ }^{52}$ and anisotropic spins, ${ }^{47,80}$ quantized vibrations, ${ }^{9,81}$ and nuclear spins ${ }^{15,82-84}$ ). In particular, electronic, ${ }^{37,39,40,42,43,57,59,85}$ spin-orbit ${ }^{62,86}$ structure as well as electro-mechanical coupling $33,44,64,87$ of CNTs has been very accurately modeled this way.
In molecular electronics, transport spectroscopy takes a prominent role since imaging of the device is challenging. By moving to molecular-scale gated structures, one often compromises real-space imaging. In this paper, we highlight the advantages that such structures offer. Nevertheless, electrical gates that work simultaneously with a scanning tip ${ }^{88}$ or a $\mathrm{MCBJ}^{89}$ have been realized, but with rather low gate coupling. Notably, mechanical gating ${ }^{90-94}$ by lifting a single molecule from the substrate has been demonstrated, resulting in $\mathrm{d} I / \mathrm{d} V$ stability diagrams where the role of $V_{\mathrm{g}}$ taken over by the tipheight $z$ in Fig. 1. A scanning quantum-dot ${ }^{95}$ has also been realized using a single-molecule. ${ }^{96}$

\section{Resonant excitations-Gate dependence}

In the resonant transport regime, one considers processes of the leading order in the tunnel coupling $\Gamma$, cf. Eq. (1). Although most of this is in principle well-known, we review this approach ${ }^{72,97}$ since some of its basic consequences for the off-resonant regime - discussed below-are often overlooked.

Typically, the analysis of resonant spectra requires a model Hamiltonian $\mathcal{H}$ that involves at most tens of states in the most complex situations. ${ }^{52,70,98-101}$ Its energies $E_{i}^{N}$ are labeled by the charge number $N$ and a further quantum number (orbital, spin, vibrational) collected into an index $i$. Crucial for the following discussion is the voltage-dependence of this energy spectrum. We assume it is uniform, i.e., $\propto N$, independent of further quantum numbers $i$. This can be derived from a capacitive description of the Coulomb interactions between system and electrodes referred to as the constant interaction model. ${ }^{71,72,79,97,102}$ In this case, $E_{i}^{N}\left(V_{\mathrm{g}}, V_{\mathrm{L}}, V_{\mathrm{R}}\right)$ $=E_{i}^{N}-N\left(\alpha_{\mathrm{g}} V_{\mathrm{g}}+\alpha_{\mathrm{L}} V_{\mathrm{L}}+\alpha_{\mathrm{R}} V_{\mathrm{R}}\right)$, where $E_{i}^{N}$ are constants and $V_{\mathrm{L}}\left(V_{\mathrm{R}}\right)$ is the potential applied at the source (drain) electrode. Here, $\alpha_{\mathrm{x}}=C_{x} / C$ for $x=\mathrm{L}, \mathrm{R}$ and $\mathrm{g}$ are capacitive parameters of which only two are independent since $C:=\sum_{x} C_{x}$. In Sec. II D, we discuss corrections to thisoften good-assumption. ${ }^{103}$ Unless stated otherwise, we will set for simplicity $\alpha_{\mathrm{g}}=1$, i.e., the negative shift of the energy levels equals the gate voltage. The bias is applied to the electron source, $V_{\mathrm{L}}=-V$, and the drain is grounded, $V_{\mathrm{R}}=0$, giving $E_{i}^{N}\left(V_{\mathrm{g}}, V\right)=E_{i}^{N}-N \alpha_{\mathrm{g}} V_{\mathrm{g}}+N \alpha_{\mathrm{L}} V$ and $\mu_{\mathrm{L}}=\mu_{\mathrm{R}}+V$ with constant $\mu_{\mathrm{R}}$. Unless stated otherwise, schematics are drawn assuming $\alpha_{\mathrm{L}}=1 / 2$, corresponding to symmetric and dominant source-drain capacitances $C_{\mathrm{L}}=C_{\mathrm{R}} \gg C_{\mathrm{g}}$.

The Hamiltonian for the complete transport situation takes the generic form $\mathcal{H}^{\text {tot }}:=\mathcal{H}+\mathcal{H}^{\text {res }}+\mathcal{H}^{\mathrm{T}}$, where $\mathcal{H}^{\mathrm{T}}$ is a sum of tunneling Hamiltonians that each transfers a single electron across one of the junctions to either metal electrodes. The electrodes, labeled by $r=\mathrm{L}$ (left) and R(right), are described by $\mathcal{H}^{\text {res }}$ —essentially through their densities of states - and by their electrochemical potentials $\mu^{r}$ and temperature $T$. For the present purposes, this level of detail suffices, e.g., see Ref. 70 for details. For a tunneling process involving such a transfer of precisely one electron, one of the electrochemical potentials has to fulfill

$$
\mu^{r} \geq E_{f}^{N+1}-E_{i}^{N} \quad \text { for } \quad r=\mathrm{L}, \mathrm{R},
$$

in order for the electron to be injected into an $N$-electron state $i$, resulting in the final $N+1$-electron state $f$. Below this threshold the state $(N+1, f)$ is unstable, i.e., it decays back to $(N, i)$ 
by expelling the electron back into the electrode. The rate for the injection process, $W_{f, i}^{N+1, N}$, is given by familiar Golden Rule expressions and depends on the difference of both sides of Eq. (2) relative to temperature $T$. When the process turns on by changing $V$, it gives rise to a peak in the differential conductance, $\mathrm{d} I / \mathrm{d} V$, corresponding to a sharp step in current, of width $T$ and height $\sim \Gamma / T \ll 1$ (in units of $e^{2} / h$ ) since we are assuming weak coupling and high temperature.

If the total system conserves both the spin and its projection along some axis (e.g., the $B$-field axis), the rate involves a selection-rule-governed prefactor. This prefactor is zero unless the change of the molecular spin and its projection satisfy

$$
|\Delta S|=1 / 2 \quad \text { and } \quad|\Delta M|=1 / 2 .
$$

These conditions reflect the fact that only a single electron is available for transferring spin to the molecule.

Incidentally, we note that this picture is very useful even beyond the weak couplings and high temperatures assumed here. Close to the resonance defined by condition (2), the transport still shows a peak which is, however, modified by higher-order corrections. The width of the current step becomes broadened $\propto \Gamma$, giving a conductance peak $\sim 1$ in units of $e^{2} / h$. Its energy position may shift on the order of $\Gamma$.

It is now clear in which regime of applied voltages the above picture applies. In Fig. 3, this is sketched in the plane of applied bias $(V)$ and gate voltage $\left(V_{\mathrm{g}}\right)$. Here, we call such a (schematic) $\mathrm{d} I / \mathrm{d} V$ intensity plot-also known as stability diagram or Coulomb-diamond-a transport spectrum. The

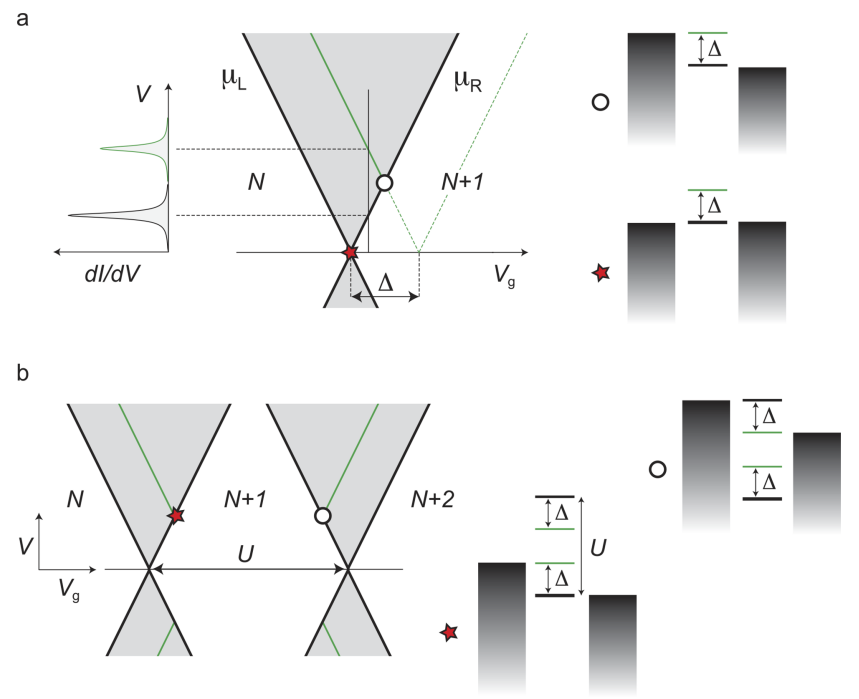

FIG. 3. Resonant regime: main features (a) current flows in the bias window set by Eq. (4) (shaded) for two charge states $N$ and $N+1$. The boundary lines (bold), where $\mu_{r}=E_{g}^{N+1}-E_{g}^{N}$ for $r=\mathrm{L}, \mathrm{R}$, have slopes $-\alpha_{\mathrm{g}} /\left(1-\alpha_{\mathrm{R}}\right)$ and $\alpha_{\mathrm{g}} / \alpha_{\mathrm{R}}$, respectively, allowing the capacitive parameters to be determined. The green lines, offset horizontally by $\Delta=E_{e}^{N+1}-E_{g}^{N+1}$, indicate the window of accessibility of the excited state $E_{e}^{N+1}$ and are defined by $\mu_{r}=\Delta+E_{g}^{N+1}-E_{g}^{N}$. (b) Similar to figure (a), for three charge states $N, N+1$, and $N+2$. This adds a copy of the bias window of (a) that is horizontally offset by the energy $U$ [Eq. (6)] with boundaries $\mu_{r}=E_{g}^{N+2}-E_{g}^{N+1}$ for $r=\mathrm{L}, \mathrm{R}$. The excitation lines on the right (green) are mirrored horizontally, $\mu_{r}=-\Delta+E_{g}^{N+2}-E_{g}^{N+1}$ for $r=\mathrm{L}, \mathrm{R}$, since electron processes relative to $N+1$ have become hole processes. indicated vertical linecuts through this diagram correspond to $\mathrm{d} I / \mathrm{d} V$ traces measured in STM or MCBJ experiments. Applied to the ground states of subsequent charge states-labeled by $g$-Eq. (2) gives the two inequalities

$$
\mu^{L} \geq E_{g}^{N+1}-E_{g}^{N} \geq \mu^{R} .
$$

These define the shaded bias window in Fig. 3(a), delimited by the cross. Here, a single electron entering from the left can exit to the right, resulting in a net directed current.

It is now tempting to naively define the off-resonant regime as the complement of the grey resonant regime in Fig. 3(a), i.e., by moving across its boundaries by more than $T$ or $\Gamma$. A key point of our paper is that this simple rationale is not correct already for a small finite bias matching some excitation at energy $\Delta$, indicated by green lines in Fig. 3(a). Only in the linear-response regime ${ }^{102}$ around $\mu=\mu_{\mathrm{L}}=\mu_{\mathrm{R}}$, the offresonant regime can be defined as the complement of the resonant regime

$$
\left|E_{g}^{N+1}-E_{g}^{N}-\mu\right| \gg \max \{\Gamma, T\}
$$

In subsequent charge states analogous considerations apply: transitions between charge states $N+1$ and $N+2$ give rise to a shifted copy of the bias window as shown in Fig. 3(b). The shift_experimentally directly accessible_-is denoted by

$$
U:=\left(E_{g}^{N+2}-E_{g}^{N+1}\right)-\left(E_{g}^{N+1}-E_{g}^{N}\right) .
$$

This includes the charging energy of the molecule, but also the magnitude of orbital energy differences and the magnetic field. For example, for a single orbital level with charging energy $u>0$ and magnetic field $B$ one finds $U=u+|B|>u$ due to the opposite spin-filling enforced by the Pauli principle.

\section{Stationary state and resonant transport current}

The above rules are substantiated by a simple master equation for the stationary-state occupations $P_{i}^{N}$ of the states with energy $E_{i}^{N}$ that can be derived from the outlined model, see, e.g., Ref. 70. This approach is used in Sec. III C 2 to model part of our experiment. For the $N \leftrightarrow N+1$ resonance regime, the stationary-state equation reads (for notational simplicity, we here $\operatorname{set} N=0$ )

$$
\frac{\mathrm{d}}{\mathrm{d} t}\left[\begin{array}{l}
\mathbf{P}^{0} \\
\mathbf{P}^{1}
\end{array}\right]=0=\left[\begin{array}{ll}
\mathbf{W}^{0,0} & \mathbf{W}^{0,1} \\
\mathbf{W}^{1,0} & \mathbf{W}^{1,1}
\end{array}\right]\left[\begin{array}{l}
\mathbf{P}^{0} \\
\mathbf{P}^{1}
\end{array}\right] .
$$

Here, $\mathbf{W}^{1,0}$ is the matrix of transition rates $W_{f, i}^{1,0}$ between states $(0, i)$ and $(1, f)$ and analogously for $\mathbf{W}^{0,1}$. For example, one of the equations,

$$
\frac{\mathrm{d}}{\mathrm{d} t} P_{f}^{1}=\sum_{i} W_{f, i}^{1,0} P_{i}^{0}+W_{f, f}^{1,1} P_{f}^{1},
$$

describes the balance between the gain in occupation probability due to all transitions $(0, i) \rightarrow(1, f)$ and the leakage $-W_{f, f}^{1,1}$ from the state $(1, f)$. The entries of the diagonal matrices $\mathbf{W}^{0,0}$ and $\mathbf{W}^{1,1}$ have negative values $W_{f, f}^{0,0}=-\sum_{i} W_{i, f}^{1,0}$ and $W_{f, f}^{1,1}=-\sum_{i} W_{i, f}^{0,1}$, respectively, such that probability normalization $\sum_{i} P_{i}^{0}+\sum_{j} P_{j}^{1}=1$ is preserved in Eq. (7). In the leading order in $\Gamma$, the rate matrix has separate contributions from the left $(r=\mathrm{L})$ and right $(r=\mathrm{R})$ electrodes: $\mathbf{W}=\mathbf{W}^{\mathrm{L}}+\mathbf{W}^{\mathrm{R}}$. 
These allow the stationary current to be computed by counting the electrons transferred by tunnel processes through the $r$ th junction,

$$
I^{r}=\sum_{N_{f}, N_{i}}^{\prime} \sum_{f, i}\left(N_{f}-N_{i}\right) \times\left[W^{r}\right]_{f, i}^{N_{f}, N_{i}} P_{i}^{N_{i}},
$$

where stationarity guarantees $I^{\mathrm{L}}=-I^{\mathrm{R}}$. We note that because we are considering only single-electron tunneling processes (first order in $\Gamma$ ), the primed sum is constrained to $N_{f}=N_{i} \pm 1$ by charge conservation.

\section{B. Off-resonant transport spectroscopy}

We now take the opposite point of view and consider transport entirely due to virtual charging or scattering through the molecule. The resulting off-resonant transport spectroscopy, alternatively called cotunneling (COT) spectroscopy or IETS, dates back to Lambe and Jacklevic. ${ }^{104}$ The discussion of the precise conditions under which the off-resonant picture applies is postponed to Sec. II C. Throughout we will denote by the label COT—unless stated otherwise-inelastic cotunneling.

The attractive feature of off-resonant relative to resonant spectroscopy is the higher energy resolution as we explain below [Eq. (18) ff.]. Exploiting this in combination with the STM's imaging capability has allowed chemical identification. ${ }^{23,26,27,96,105-112}$ This in turn has enabled atomistic modeling of the junction using $a b$ initio calculations, ${ }^{113-116}$ also including strong interaction effects, ${ }^{92,117,118}$ giving a detailed picture of transport on the atomic scale. ${ }^{119-125}$

In recent years, off-resonant spectroscopy has been also intensively applied to spin systems ${ }^{18,23,25,126-128}$ in more symmetric $^{129}$ STM configurations. However, it is sometimes not realized that the same off-resonant spectroscopy also applies to gated molecular junction, and more generally to QDs. ${ }^{62,130-132}$ In fact, motivated by the enhanced energy resolution, spectroscopy of discrete spin-states was introduced in gate-controlled semiconductor QDs ${ }^{130,133,134}$ before it was introduced in STM as spin-flip spectroscopy,${ }^{18}$ see also Refs. 135 and 136. COT spectroscopy is also used to study molecular properties other than spin, e.g., vibrational states. ${ }^{137-142}$

\section{Off-resonant excitations-No gate dependence}

In the off-resonant picture, one considers transport due to next-to-leading order processes, i.e., of order $\Gamma^{2}$ in the tunnel rates. This involves elastic (inelastic) processes involving two electrons from the electrodes and a zero (net) energy transfer of energy. When the maximal energy supplied by the electronsone electron coming in from, say, $r=\mathrm{L}$ at high energy $\mu_{\mathrm{L}}$, and the other outgoing to $r=\mathrm{R}$ at low energy $\mu_{\mathrm{R}}$ - exceeds a discrete energy difference of the molecule,

$$
\mu_{\mathrm{L}}-\mu_{\mathrm{R}}=V \geq \Delta_{f, i}^{N+1}:=E_{f}^{N+1}-E_{i}^{N+1},
$$

transport may be altered with $V$. Importantly, on the right hand side, all $V$ and $V_{\mathrm{g}}$ dependences of the energies cancel out [cf. Sec. II A 1] since we assumed that the applied voltages uniformly shift the excitation spectrum for fixed charge. ${ }^{103,143}$

The occurrence of such a process depends on whether the initial state $i$ is occupied or not by another already active process. It thus depends on whether we are in the "equilibrium" or "nonequilibrium" regime, both of which are accessible in our experiment in Sec. III. The spectroscopy rules require the following separate discussion.

a. "Equilibrium" inelastic COT. Already in the linear transport regime, $V \lesssim T, \Gamma$ (assuming no excitations lie below $T$ and $\Gamma$ ), here is scattering through the molecule in a fixed stable charge state in the form of elastic COT, ${ }^{135,136,144}$ see Table I for the varied nomenclature. This gives rise to a small current scaling $\propto \Gamma^{2}$. With increasing bias $V$, this mechanism yields a nonlinear background current which is, however, featureless.

When the voltage provides enough energy to reach the lowest excitation $e$ of the $N+1$-electron ground state $g$, the transition $(N+1, g) \rightarrow(N+1, e)$ is enabled, cf. Fig. 2(c). This occurs when the gate-voltage independent criterion set by Eq. (10) with $i=g$ and $f=e$ is satisfied,

$$
V \geq \Delta_{e, g}^{N+1} .
$$

The above energy condition is the tell-tale sign of an offresonant process: as sketched in Fig. 4(a), this allows for a clear-cut distinction from resonant processes with a gate dependent energy condition (2). Importantly, such a COT feature always connects to the gate-dependent SET resonance

a

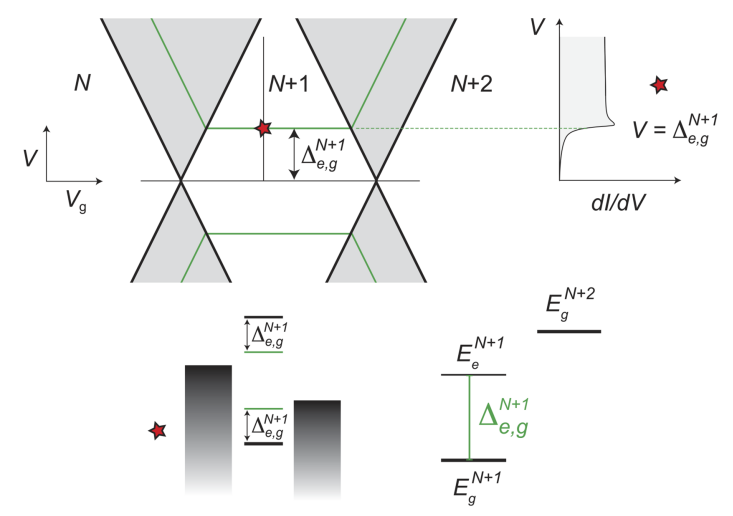

b

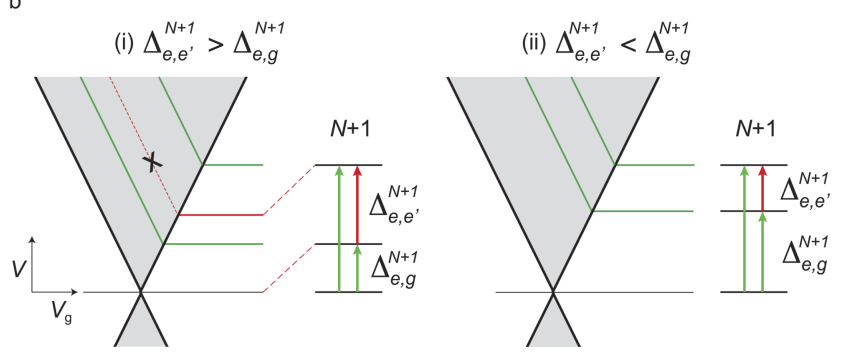

FIG. 4. COT transport conditions. (a) Same as Fig. 3(b), now indicating the "equilibrium" resonance (green horizontal line) at which the excitation $(N$ $+1, e)$ is reached from the ground state $(N+1, g)$ by a COT process. This horizontal line always connects to a SET resonance $(N, g) \rightarrow(N+1, e)$ on the left $[(N+2, g) \rightarrow(N+1, e)$ on the right] (green tilted lines). At the point where the COT and SET resonance lines meet two conditions are simultaneously satisfied: one for the onset of SET, electrochemical potential $=\mu_{\mathrm{L}}=E_{g}^{N+1}-E_{g}^{N}$ $\left[\mu_{\mathrm{R}}=E_{g}^{N+2}-E_{g}^{N+1}\right]$, and one for onset of inelastic COT, bias $=\mu_{\mathrm{L}}-\mu_{\mathrm{R}}$ $=E_{e}^{N+1}-E_{g}^{N+1}=$ excitation at fixed $N+1$. This is depicted in Fig. 3(b) where the chemical potential diagrams are sketched for these two crossing points, marked $\circ$ and $\star$ in that figure. (b) "Nonequilibrium" COT resonance corresponding to a transition $(N+1, e) \rightarrow\left(N+1, e^{\prime}\right)$ starting from an excited state efor $N+1$ electrons. Case (i) and (ii) are discussed in the text. This resonance (red horizontal line) does not connect to some SET resonance (crossed-out dashed red line) since there is no single-electron transition $(N, e) \rightarrow\left(N+1, e^{\prime}\right)$ : the state $e$ is an excitation for $N+1$ electrons, not for $N$ electrons. This should be contrasted with "equilibrium" COT resonances in (a). 
corresponding to excitation $\Delta_{e, g}^{N+1}$. As in the resonant regime, we stress that criterion (11) uses the peak position in the $\left(V_{\mathrm{g}}, V\right)$ plane as a primary indicator. The line shape along a vertical cut in the figure, as measured in STM, may be less clear. In theoretical modeling, the line shape is also not a unique indicator. The line shape is a good secondary indicator of the nature of a process.

b. "Nonequilibrium" inelastic COT: Electronic pump-probe spectroscopy. The above "equilibrium" picture of off-resonant transport has been successfully applied in many instances. However, as the first excited state $(N+1, e)$ is accessed, the rules of the game change. If the relaxation induced by sources other than transport is weak enough, ${ }^{145}$ the occupation of the excited states can become non-negligible. In such a case, as illustrated in Fig. 4(b), a secondary inelastic COT process from the excited state $e$ to an even higher excited state $e^{\prime}$ should be considered. Such secondary processes, with the generic condition

$$
V \geq \Delta_{e^{\prime} e}^{N+1}=\Delta_{e^{\prime} g}^{N+1}-\Delta_{e g}^{N+1},
$$

indicate a device with an intrinsic relaxation rate small compared to COT rates $\propto \Gamma^{2}$. As discussed in Fig. 4(b), such excitations never connect to a corresponding SET excitation in the transport spectrum. At this point, two cases have to be considered, both of which are relevant to our experiment in Sec. III D.

(i) If $\Delta_{e^{\prime} e}^{N+1}>\Delta_{e g}^{N+1}$, i.e., the gaps in the energy spectrum grow with energy, an extra "nonequilibrium" inelastic COT resonance at bias $V=\Delta_{e^{\prime} g}^{N+1}-\Delta_{e g}^{N+1}$ appears, as illustrated in panel (i) of Fig. 4(b). This extra resonance is very useful since it provides a further consistency check on the excitations $\Delta_{e g}^{N+1}$ and $\Delta_{e^{\prime} g}^{N+1}$ observed independently in the SET. (If the SET transition to $e^{\prime}$ is not allowed by a selection rule, the secondary COT resonance may be the only evidence of this state.) Clearly, the intensity of such secondary "nonequilibrium" COT resonances is generally expected to be lower than the primary ones that start from the ground state. In Sec. III D, we will experimentally control this sequential COT electronic pump-probe excitations by tuning a magnetic field.

(ii) In the opposite case, $\Delta_{e^{\prime} e}^{N+1}<\Delta_{e g}^{N+1}$, no extra COT excitation related to $e^{\prime}$ appears: there is no change in the current at the lower voltage $\Delta_{e^{\prime} e}^{N+1}$ because the initial state $(N$ $+1, e)$ only becomes occupied at the higher voltage $\Delta_{e g}^{N+1}$. This is illustrated in panel (ii) of Fig. 4(b). Examples of both these cases occur in the off-resonant spectra of molecular magnets due to the interesting interplay of their easy-axis and transverse anisotropy, see the supplement of Ref. 48.

\section{Stationary state and off-resonant transport current}

Similar to the resonant case, the conditions (10)-(12) are incorporated in a simple stationary master equation for offresonant transport whose derivation we discuss further below. In particular, the occupation probabilities $P^{N+1}$ in the stationary transport state are determined by (as previously, we put $N=0$ )

$$
\frac{\mathrm{d}}{\mathrm{d} t} \mathbf{P}^{1}=0=\mathbf{W}^{1,1} \mathbf{P}^{1} .
$$

Here, $\mathbf{W}^{1,1}$ is a matrix of rates $W_{f, i}^{1,1}$ for transitions between states $i \rightarrow f$. Since in the off-resonant regime charging is only virtual, these transitions now occur for a fixed charge state. The matrix takes the form $\mathbf{W}^{1,1}=\sum_{r r^{\prime}} \mathbf{W}^{1,1 ; r, r^{\prime}}$, including rate matrices $\mathbf{W}^{1,1 ; r, r^{\prime}}$ for back-scattering from the molecule (to the same electrode, $r=r^{\prime}$ ) and scattering through it (between electrodes $r \neq r^{\prime}$ ). The current is obtained by counting the net number of electrons transferred from one electrode to the other,

$$
I^{\mathrm{L} \rightarrow \mathrm{R}}=\sum_{f, i}\left(W_{f, i}^{1,1 ; \mathrm{R}, \mathrm{L}}-W_{f, i}^{1,1 ; \mathrm{L}, \mathrm{R}}\right) P_{i}^{1}
$$

The inclusion into this picture of the above discussed "nonequilibrium" COT effects depends whether one solves the master equation (13) or not. To obtain the simpler description of "equilibrium" inelastic COT [case (i) above], one can insert by hand equilibrium populations $P_{i}^{1}=e^{-E_{i}^{1} / T} / \mathcal{Z}^{1}$ directly into Eq. (14). Solving, instead, Eq. (13) without further assumptions gives the "nonequilibrium" inelastic COT case $^{25,146}$ discussed above [case (ii)]. In practice, these two extreme limits-both computable without explicit consideration of intrinsic relaxation-are always useful to compare since any more detailed modeling of the intrinsic relaxation will lie somewhere in between.

The electron tunneling rates in Eq. (13) are made up entirely of contributions of order $\Gamma^{2}$. There are two common ways of computing these rates, and we now present the underlying physics relevant for the discussion in Sec. II C.

a. Appelbaum-Schrieffer-Wolff Hamiltonian. A conceptual connection between the off-resonant virtual charging picture and the resonant picture of real charging in Sec. II A emerges naturally when applying the unitary transformation ${ }^{147}$ due to Appelbaum, ${ }^{148,149}$ and Schrieffer and Wolff ${ }^{150-152}$ (ASW) to the transport Hamiltonian $\mathcal{H}^{\text {tot }}$ [cf. Sec. II A]. The effective ASW model obtained in this way allows one to easily see the key features of the off-resonant spectroscopy.

In this approach, the one-electron tunneling processes described by the Hamiltonian $\mathcal{H}^{T}$ are transformed away and the charge state is fixed by hand to a definite integer. With that, also all the gate-voltage dependence of resonance positions [Eq. (11) ff.] drops out. This new ASW model is obtained by applying a specially chosen unitary transformation $\mathcal{U}$ to the original Hamiltonian such that

$$
\begin{aligned}
\mathcal{H}^{\text {tot }} & \rightarrow \mathcal{U}\left(\mathcal{H}+\mathcal{H}^{\mathrm{R}}+\mathcal{H}^{\mathrm{T}}\right) \mathcal{U}^{\dagger} \\
& \approx \mathcal{H}+\mathcal{H}^{\mathrm{R}}+\mathcal{H}^{\mathrm{A}}+\mathrm{O}\left(\Gamma^{4}\right) .
\end{aligned}
$$

The single-electron coupling $\mathcal{H}^{\mathrm{T}} \propto \sqrt{\Gamma}$ is effectively replaced by $\mathcal{H}^{\mathrm{A}} \propto \Gamma$, which involves only two-electron processes. When restricted to fixed charge $N$ on the molecule, $\mathcal{H}^{\mathrm{A}}$ exclusively represents scattering of electrons off and through the molecule. In many cases of interest, it contains terms describing the potential (scalar) and exchange (spin-spin) scattering of electrons with amplitudes $J$ and $K$, respectively. For example, for a single-orbital model, 


$$
\mathcal{H}^{\mathrm{A}}=\sum_{r, r^{\prime}}\left(J_{r r^{\prime}} \mathbf{S} \cdot \mathbf{s}_{r r^{\prime}}+K_{r r^{\prime}} N n_{r r^{\prime}}\right)
$$

where the operators $s_{r r^{\prime}}\left(n_{r r^{\prime}}\right)$ describe spin-(in)dependent intra- $\left(\left[r=r^{\prime}\right]\right)$ and inter-electrode $\left(r \neq r^{\prime}\right)$ scattering of electrons. See Ref. 97 for details, such as the energy-dependence of $J$ and $K$ ignored above.

Selection rules. The ASW coupling $\mathcal{H}^{\mathrm{A}}$ has selection rules that differ from the original single-electron tunnel coupling $\mathcal{H}^{\mathrm{T}}$. If the time-evolution in the virtual intermediate state conserves spin $(M$ and $S)$, then the selection rules for $\mathcal{H}^{\mathrm{T}}$ imply the following selection rules for transition rates obtained from $\mathcal{H}^{\mathrm{A}}$ :

$$
|\Delta S|=0,1 \quad \text { and } \quad|\Delta M|=0,1 .
$$

For this to be valid, both the molecule $(\mathcal{H})$ and electrodes $\left(\mathcal{H}^{\mathrm{R}}\right)$ must be spin-isotropic. However, even when effects that break this spin-isotropy are present, these selection rules may still hold to good approximation. This requires the magnetic field, magnetic anisotropy terms, etc., to have characteristic energies that are small relative to the distance to resonance. In these cases, the selection rules (17) reflect that the two ${ }^{153}$ electrons involved in the scattering process have integer spin 0 or 1 available for exchange with the molecule. We will apply this in Sec. III A.

For the example case (16), the scattering is coupled to the molecule only through its charge ( $N$, constant) and spin $(\mathbf{S})$. As a result, the rules (17) apply with $\Delta S=0$ and $|\Delta M|=0$ (if $S=0$ ) or $|\Delta M|=1$ (if $S=1 / 2$ ) because ${ }^{154}$ a single orbital does not support a triplet spin $(S=1)$. For more general situations, $\mathcal{H}^{\mathrm{A}}$ contains additional terms ${ }^{155}$ that change both spin and orbital occupations, see also Sec. 4.3 of Ref. 156. These do allow for $|\Delta S|=1$ while still larger changes are forbidden. Physically, one can understand that $|\Delta S|=1$ is indeed possible ${ }^{146,157}$ for a two-orbital molecule: starting from a singlet $S$ $=0$, one can reach spin triplet $S=1$ via a virtual intermediate state with spin $S^{\prime \prime}=1 / 2$, i.e., when, after exiting, an electron returns into a different orbital. This effectively moves an electron between the two orbitals, allowing for a singlet-triplet transition.

Lifetime. After transforming to this new effective picture, scattering becomes the leading order transport mechanism. The Golden Rule approach can be then applied analogously to the case of the resonant regime, but now with respect to the ASW scattering $\mathcal{H}^{\mathrm{A}}$. In this way, Eq. (13) is obtained together with an expression for the corresponding rate matrix $W^{1,1}$. The $\mathrm{d} I / \mathrm{d} V$ given by Eq. (14) shows gate-voltage-independent steps at energies set by Eq. (10).

Although at high temperatures these steps get thermally broadened, ${ }^{104}$ at low enough $T$ their broadening is smaller than that of the SET peaks. While calculation of this line shape requires higher-order contributions to $W^{1,1}$, the relevant energy scale (inverse lifetime) is given by the magnitude of the Golden Rule rates for the effective coupling $\mathcal{H}^{\mathrm{A}}$ scaling as

$$
\left(\mathcal{H}^{\mathrm{A}}\right)^{2} \propto \Gamma^{2} .
$$

This results in a much larger lifetime compared to the one from SET [cf. Eq. (1)] due to the role of the interactions on the molecule suppressing charge fluctuations. The smaller intrinsic broadening is a key advantage of COT vs. SET spectroscopy. ${ }^{130}$

Line shape. Due to nonequilibrium effects, i.e., the voltage-dependence of the occupations obtained by solving Eq. (13), a small peak can develop on top of the COT step. ${ }^{133,134,146,158}$ Moreover, processes beyond the leadingorder in $\mathcal{H}^{\mathrm{A}}$, which is all the COT approach accounts for, can have a similar effect. These turn the off-resonant tunneling step into a $\mathrm{d} I / \mathrm{d} V$ peak and are in use for more precise modeling of experiments. ${ }^{25,159,160}$ Spin-polarization ${ }^{161}$ and spin-orbit effects, ${ }^{132,160}$ however, also affect the peak shape and asymmetry.

At low temperatures and sufficiently strong coupling, a nonequilibrium Kondo effect develops which has been studied in great detail. ${ }^{146,162-165}$ These works show that the peak amplitude is then enhanced nonperturbatively in the tunnel coupling, in particular, for low lying excitations. This requires nonequilibrium renormalization group methods beyond the present scope and we refer to various reviews. ${ }^{166-171}$ In particular, it requires an account of the competition between the Kondo effect and the current-induced decoherence ${ }^{172}$ in the (generalized) quantum master equation for the nonequilibrium density operator. ${ }^{164,165}$

From the present point of view of spectroscopy, the Kondo effect can be considered as a limit of an inelastic COT feature at $V=\Delta$ as $\Delta \rightarrow 0$, see Fig. 4(a). Its position is simply $V$ $=0$ at gate voltages sufficiently far between adjacent SET resonances by criterion (5). In particular, for transport spectroscopy of atomic and molecular spin systems, the Kondo effect and its splitting into COT features ${ }^{13,173-175}$ are very important especially in combination with strong magnetic anisotropy. ${ }^{176-188}$ We refer to reviews on $\mathrm{STM}^{24,189-191}$ and $\mathrm{QD}^{56}$ studies.

b. Golden Rule $\mathcal{T}$-matrix rates. A second way of arriving at the master equation (13) and the rates in $W^{1,1}$ is the socalled $\mathcal{T}$-matrix approach. ${ }^{195}$ In essence, here COT is regarded as a scattering process: in the Golden Rule, the next-to-leading order $\mathcal{T}$-matrix, ${ }^{97}$

$$
\mathcal{T}(E) \approx \mathcal{H}^{\mathrm{T}} \frac{1}{E-\mathcal{H}-\mathcal{H}^{\mathrm{res}}} \mathcal{H}^{\mathrm{T}}+\ldots
$$

is used instead of the coupling $\mathcal{H}^{T}$, where $E$ is the scattering energy. The main shortcoming of this approach is that the $\mathcal{T}$-matrix rates so obtained are infinite. The precise origin of the divergences was identified in Ref. 192 to the neglect of contributions that formally appear in first-order in $\Gamma$ but which effectively contribute only in second order to the stationary state. ${ }^{70}$ These come from the so-called secular contributions, involving off-diagonal elements of the density matrix in the energy basis, in addition to the diagonal elements, the probabilities. By taking these contributions consistently into account, ${ }^{192}$ finite effective rates ${ }^{193}$ for the master equation for the probabilities are obtained. In both the ASW and $\mathcal{T}$-matrix approach, these contributions are ignored and, instead, finite expressions for the rates are obtained only after ad-hoc infinite subtractions. ${ }^{99,194}$ This regularization "by hand" can—and in practice does-lead to rates different from the consistently computed finite rates, see Ref. 192 for explicit comparisons. These problems have also been 
related ${ }^{196}$ to the fact that the calculation of stationary transport using a density matrix (occupations) is not a scattering problem - although it can be connected to it ${ }^{197}$-in the following sense: the coupling to the electrodes is never adiabatically turned off at large times (i.e., there is no free "outgoing state").

As we discuss next, such a consistent first plus second order approach is not only technically crucial but this also leads to additional physical effects that we measure in Sec. III.

\section{Resonant-off-resonant crossover}

Having reviewed the two prominent, complementary pictures of transport due to real and virtual charging, we now turn to the crossover regime where these two pictures coexist. This has received relatively little attention, but our experiment in Sec. III highlights its importance. As we have seen, despite the fact that charging is only virtual, an energy exchange between molecule and scattering electrons can occur. Depending on the energy-level positions, this virtual tunneling can "heat" the molecule so as to switch on real charging processes even well outside the resonant regime. However, in contrast to real heating, which leads to smearing of transport features, this nonequilibrium effect actually results in sharp features in the transport as a function of bias voltage. It thus becomes a new tool for spectroscopy.

\section{SET mirages of COT excitations}

We first consider the simple case of a single excited state at energy $E_{e}^{N+1}=E_{g}^{N+1}+\Delta$ for $N+1$ electrons. In Fig. 5(a), we see that the resulting COT resonance at $V=\Delta$ (red) connects to the excited-state SET resonance $\mu_{\mathrm{L}}=\Delta+E_{g}^{N+1}-E_{g}^{N}$ (blue), see also Fig. 4(a). The other SET resonance condition for the excited state,

$$
\mu_{\mathrm{R}}=\Delta+E_{g}^{N+1}-E_{g}^{N},
$$

defines the green line dividing the inelastic COT regime $V \geq \Delta$ into two regions shaded red and blue. In the one shaded blue, at the point marked with a circle, the excited state created by a COT process is stable, that is, it cannot decay by a singleelectron process since $\Delta+E_{g}^{N+1}-E_{g}^{N}<\mu_{\mathrm{R}}$. As shown in the right panel of Fig. 5(b), the relaxation of this stable state can then only proceed by another COT process-via virtual charging - and it is thus slow $\left(\propto \Gamma^{2}\right)$. Essentially, this means that the molecule is not "hot" enough to lift the Coulomb blockade of the excited state.

In contrast, in the red shaded area, at the point marked with a star, this stability is lost as $\Delta+E_{g}^{N+1}-E_{g}^{N}>\mu_{\mathrm{R}}$. Now the relaxation proceeds much faster through a singleelectron process (order $\Gamma$ ) as sketched in the left panel of Fig. 5(b). The molecule gets charged for real (either $N$ or $N+2$ ) and quickly absorbs/emits an electron returning to the stable $N+1$ electron ground state, where the system idles waiting for the next COT excitation. Notably, this quenching of the excited state takes place far away from the resonant transport regime in terms of the resonance width, i.e., violating the linear-response criterion (5) for being offresonance.

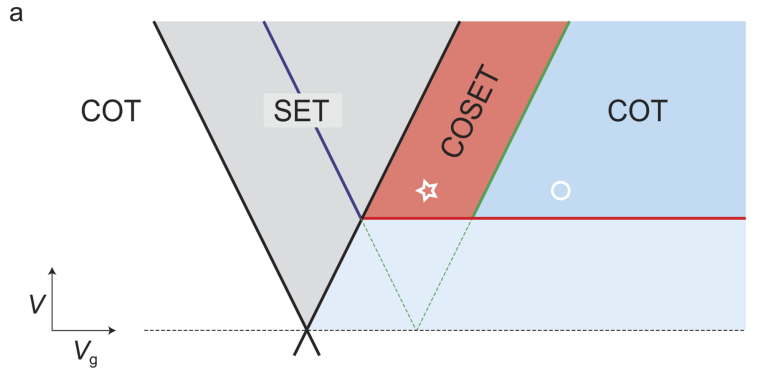

b
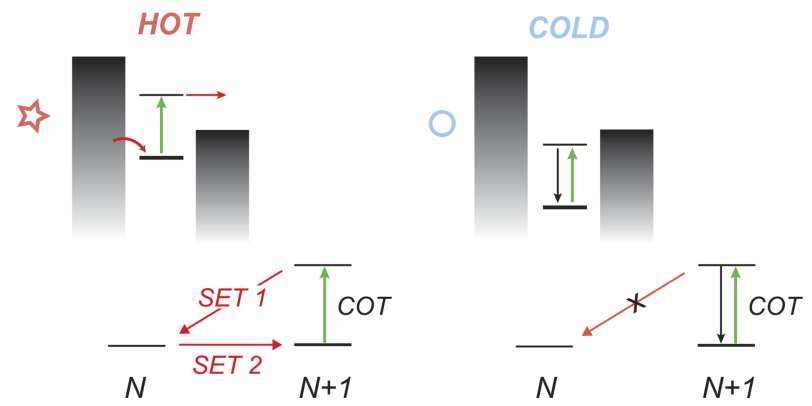

FIG. 5. Crossover regime between resonant and off-resonant transport. (a) Same as Fig. 3(a), now indicating the regimes where the excited state $(N$ $+1, e)$ relaxes by COT (darker blue area) to $(N+1, g)$ or by SET (red area) to $(N, g)$. Only such "equilibrium" COT resonances, i.e., involving the ground state $(N+1, g)$ can exhibit such a COSET mirage. The reason is that only such a type of COT resonance connects to a SET resonance as explained in Fig. 4(b) for the case of exciting the molecule while charging it, $(N, g)$ $\rightarrow(N+1, e)$. The COSET resonance corresponds to the SET transition between the same two states but in the reverse direction, relaxing the molecule $(N+1, e)$ $\rightarrow(N, g)$, while discharging it. (b) Two relaxation mechanisms after excitation by COT: Left panel: relaxation in two steps (red) via real occupation of charge state N. Right panel: when the process "SET 1" is energetically not allowed excitation (green) and relaxation (black) proceeds in a single step by COT, using charge state $N$ only virtually. (Since here process "SET 2" irrelevant it is not indicated.)

The enhanced relaxation induced by first-order tunneling, occurring when moving from the circle to the star in Fig. 5(a), leads to a change in current if no other processes (e.g., phonons and hyperfine coupling) dominate this relaxation channel $(\propto \Gamma)$. As a result, the presence of such a resonance signals a "good" molecular device, i.e., one in which the intrinsic relaxation is small compared to the transport coupling $\Gamma$. We refer to this resonance, first pointed out in Refs. 65, 130, and 133 and studied further, ${ }^{63,66,68-70}$ as cotunneling-assisted SET or COSET.

The COSET resonance has both COT and SET characters. On the one hand, the geometric construction in Figs. 5(a) and 6 shows that it stems from the same excitation as the COT step at $V=\Delta$. However, its position $V^{*}$ has the same strongly gate-voltage dependence as a SET resonance, in contrast to the original COT resonance at $V=\Delta$. Yet, the COSET peak requires COT to appear and its amplitude is relatively weak, whereas the SET peak is strong and does not require COT. For this reason, the COSET peak can be seen as a mirage of the COT excitation and a mirror image of the $(N, g) \rightarrow(N+1, e)$ SET peak, as constructed in Fig. 6(a). The resulting mirrored energy conditions can easily be checked in an experiment-cf. Fig. 14-and impose constraints on spectroscopic analysis: if $\mathrm{d} I / \mathrm{d} V$ shows a resonance as a function of bias outside the SET regime, a resonance at the mirrored position inside the SET regime should be present. 


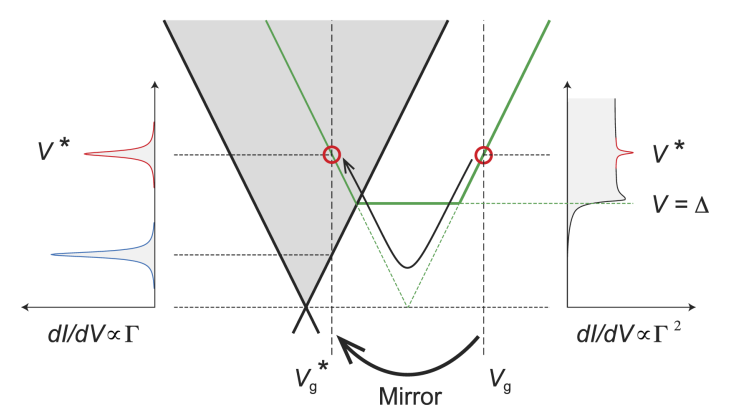

FIG. 6. Identifying a resonance as a COSET mirage. Same situation as Fig. 5(a). The vertical $\mathrm{d} I / \mathrm{d} V$ cut on the right shows a COT resonance at $V=\Delta$ and its mirage at some bias $V^{*}>\Delta$. To identify the latter as such, a corresponding SET resonance must be present at the mirrored gate voltage $V_{\mathrm{g}}^{*}$, as in the vertical cut shown on the left. Note that the indicated construction works for nonsymmetric capacitive coupling. For symmetric coupling, one can literally mirror the gate-voltage position relative to $\Delta$ on the horizontal axis.

\section{Connecting off-resonant and resonant analyses}

Besides the appearance of COSET mirages, the crossover regime provides further important pieces of spectroscopic information by constraining how SET and COT spectra continuously connect as the gate voltage is varied. This is discussed in Secs. II A 2 and II B and later on in Sec. II C 2, but we summarize the rules here. First, only "equilibrium" COT transitions can exhibit a COSET mirage as explained in Fig. 5. Second, excited-excited COT transitions (i.e., for the same charge state $N+1$ ) never connect to a corresponding SET feature, as we illustrated in panel (i) of Fig. 4(b). Finally, transitions between excited states with different charge-visible in the SET regime-never connect to a COT feature as will be illustrated in Fig. 12. These are strict consistency requirements when analyzing the transport spectra in the SET-COT crossover regime.

\section{When is transport "off-resonant"?}

We are now in the position to determine the region in which the physical picture of off-resonant scattering through the molecule of Sec. II B applies. This is illustrated in Fig. 7.

The key necessary assumption of the COT approachoften not stated precisely-is that all excited states $(N+1, e)$ that are accessible from the ground state $(N+1, g)$ must be stable with respect to first-order relaxation processes,

$$
W_{g, e}^{N, N+1}=0 \quad \text { and } \quad W_{g, e}^{N+2, N+1}=0 .
$$

This is the case if the SET condition (2) additionally holds for the excited states, i.e., for $i=e$ in Eq. (2),

$$
\mu_{r}<E_{e}^{N+1}-E_{g}^{N} \quad \text { and } \quad \mu_{r}>E_{g}^{N+2}-E_{e}^{N+1}
$$

for both $r=\mathrm{L}$, R. We note that in theoretical considerations, it is easy to lose sight of condition (22) when "writing down" an ASW Hamiltonian model (or only $\mathcal{T}$-matrix rates for COT) [Sec. II B 2] and assuming the couplings to be fitting parameters of the theory. (In fact, in addition to condition (22), one needs to check that all excited states that are accessible via nonequilibrium cascades of COT transitions ("nonequilibrium COT") are stable. We will not discuss this further complication.) a

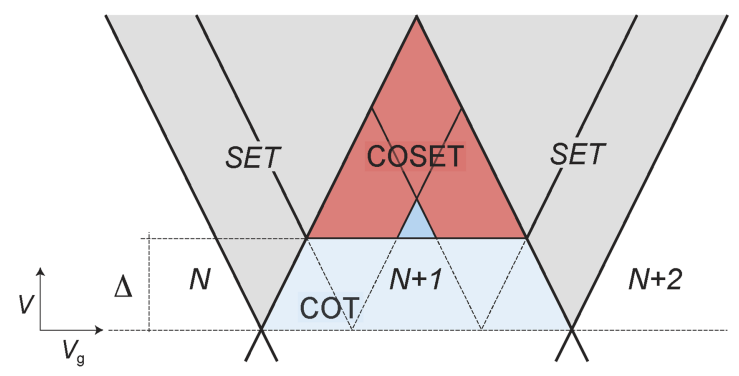

b

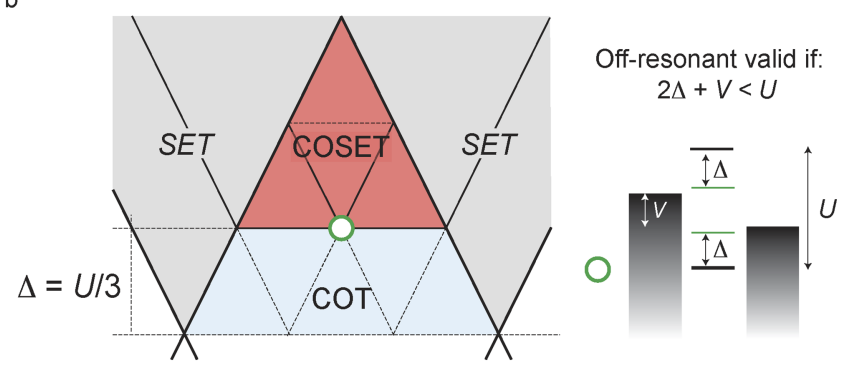

C

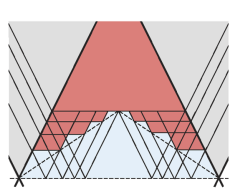

Sub-harmonic

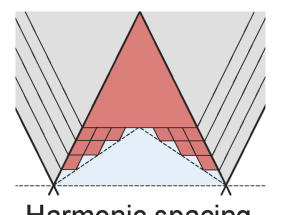

Harmonic spacing

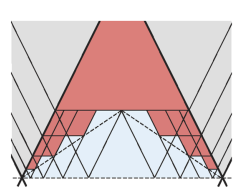

Super-harmonic
FIG. 7. Shape and size of the off-resonant regime as $\Delta$ is varied relative to $U$. (a) Off-resonant regime for an excitation $\Delta<U / 3$ as in Fig. 4(a). As in Fig. 5, the regions where the off-resonant approach is valid (fails) are colored blue (red). In the light blue region where $V<\Delta$, there is only elastic COT (dashed black construction lines are not resonances), but for $V \gtrsim \Delta$ inelastic COT does excite the molecule. The off-resonant approach only applies when both excitation and relaxation proceed by virtual charging. This is the case in the darker blue triangle which is restricted from both sides by Eq. (22) and shrinks in size with increasing $\Delta \rightarrow U / 3$. (b) Left panel: For an excitation with $\Delta=U / 3$, the off-resonant picture no longer works for the inelastic COT excitation at $V=\Delta$. Thus, the excitations $\Delta<U / 3$ for which the off-resonant picture works lie in the blue area of this figure, which amounts to $5 / 9 \approx 55 \%$ of the nominal off-resonant regime (blue plus red area). Right panel: To see why $\Delta=U / 3$ is the threshold value, we consider the best-case scenario for the off-resonant picture to work, i.e., at fixed gate voltage horizontally at the center while varying $V$ vertically, traversing the point $\circ$. The diagram shows that there is no relaxation by SET as long as the bias satisfies $2 \Delta+V<U$. Requiring this to hold at the onset of inelastic excitation by COT, $V=\Delta$, gives the threshold value. (c) Several excitations from a superharmonic (left), harmonic (center), and subharmonic (right) spectrum for charge $N+1$. In the limit of vanishing harmonic energy spacing, the blue region where the COT picture works approaches $1 / 3 \approx 33 \%$ of the nominal off-resonant regime.

In Fig. 5(a), we already shaded in light blue the region bounded by the first condition (22) where the COT picture applies. In Fig. 7(a), we now show that the full restrictions imposed by both virtual charge states $N$ and $N+2$ in (22) strongly restrict the validity regime of the COT approach for states with real occupations and charge $N+1$. In Fig. 7(b) and its caption, we explain that for any individual excitation $\Delta>U / 3$ the off-resonant picture always breaks down in the sense that it works only for elastic COT, i.e., for $V<\Delta$. This amounts to $55 \%$ of the nominal off-resonant regime.

When accounting for several excited states below the threshold $U / 3$, a sizeable fraction of this region must be further excluded. In Fig. 7(c), we construct the regime of validity (blue) for some example situations. The shape and size of this 
validity regime (light blue) depends on the details of the excitation spectrum. The center panel illustrates that for a harmonic spectrum the COT picture in fact applies in only $\sim 33 \%$ of the nominal off-resonant regime (i.e., obtained by taking the complement of the resonant regime). The left and right panels in Fig. 7(c) show how this changes for anharmonic spectra characteristic of quantum spins with positive and negative magnetic anisotropy, respectively.

In summary, resonant processes always dominate the relaxation of excitations at energy $\Delta>U / 3$ populated by off-resonant excitation because they are "too hot": for such excitations, there is no deep/far off-resonant regime where considerations based on the COT picture alone are valid. For lower-energy excitations, $\Delta \leq U / 3$, there is a triangularshaped region in which one is still truly far off-resonance and excitations are not quenched. The size of that region varies according to (22) and is much smaller than naively expected by extending the linear-response criterion (5). Although theoretical $^{65-70}$ and experimental ${ }^{63,130}$ studies on COSET exist, this point seems to have been often overlooked and is worth emphasizing. Experimentally, to be sure that the off-resonant picture applies to unidentified excitation, one must at least have an estimate of the gap $U$ and of the level position or, preferably, a map of the dependence of transport on the level position independent of the bias as in gated experiment discussed in Sec. III or STM situations allowing for mechanical gating. ${ }^{90-94}$

\section{Stationary state and current at the resonant- off-resonant crossover}

Due to their hybrid character, COSET mirages do not emerge in a picture of either real or virtual charging alone. In particular, SET processes are omitted when deriving the COT rates by means of the ASW transformation [Sec. II B], and, for this reason, that picture cannot account for these phenomena. Instead, a way to capture these effects is to extend Eqs. (7) and (13) to a master equation which simultaneously includes transition rates of leading $(\Gamma)$ and next-to-leading order $\left(\Gamma^{2}\right)$. This has been done using the $\mathcal{T}$-matrix approach, ${ }^{99,194}$ requiring the ad-hoc regularization by hand mentioned in Sec. II B 2 b. A systematic expansion which avoids these problems is, however, well-known. ${ }^{144,198,199}$ We refer to Refs. 70, 192, and 200 for the calculation of the rates and for a discussion ${ }^{192}$ of how contributions beyond weak coupling account for correlations between the molecule and electrodes.

Relevant to our experiment in Sec. III is that with the computed rates in hand, a stationary master equation needs to be solved to obtain the occupation of the states and from these the current. We stress that even when far off-resonance-where naively speaking the charge is fixed to, say, $N+1-$ a description of the transport requires a model which also includes both the $N$ and $N+2$ charge states, together with their relative excitations. This is essential to correctly account for the relaxation mechanisms that visit these states for real and not virtually. Note that keeping these states is not related to obtaining the correct strength of the couplings for scattering in the ASW Hamiltonian. Even with the correct values for $J$ and $K$ in Eq. (16), the COSET mirages are missed since $\mathcal{H}^{\mathrm{A}}$ only accounts for scattering processes. The minimal master equation required for off-resonant transport thus takes then the following form:

$$
\frac{\mathrm{d}}{\mathrm{d} t}\left[\begin{array}{l}
\mathbf{P}^{0} \\
\mathbf{P}^{1} \\
\mathbf{P}^{2}
\end{array}\right]=0=\left[\begin{array}{lll}
\mathbf{W}^{0,0} & \mathbf{W}^{0,1} & \mathbf{W}^{0,2} \\
\mathbf{W}^{1,0} & \mathbf{W}^{1,1} & \mathbf{W}^{1,2} \\
\mathbf{W}^{2,0} & \mathbf{W}^{2,1} & \mathbf{W}^{2,2}
\end{array}\right]\left[\begin{array}{l}
\mathbf{P}^{0} \\
\mathbf{P}^{1} \\
\mathbf{P}^{2}
\end{array}\right]
$$

where as before $N=0$ for simplicity. Here the rates for the various processes change whenever one of the energetic conditions (2) and (10) is satisfied. Examination of the various contributions in the expression of the rate matrices ${ }^{70}$ reveals that the following effects are included:

- $\mathbf{W}^{1,0}$ is a matrix of SET rates that change when condition (2) is met. It also includes $\Gamma^{2}$-corrections that shift and broaden the SET resonance.

- $\mathbf{W}^{1,1}$ is a matrix of both SET and COT rates. The latter change when condition (10) is met.

- $\mathbf{W}^{2,0}$ and $W^{0,2}$ are matrices of pair-tunneling rates, e.g., $W_{f, i}^{2,0}$ for transitions between states differing by two electrons, $(N, i) \rightarrow(N+2, f)$. These lead to special resonances discussed in Sec. II D.

The solution of the full stationary master equation (23) requires some care co,192 $^{7 u e}$ to the fact that it contains both small COT rates and large SET rates whose interplay produces the COSET mirages. Even though the (first-order) SET rates are large, they have a small—albeit non-negligible-effect since, in the stationary situation, the initial states for these transitions may have only small occupations. These occupations, in turn, depend on the competition between all processes/rates in the stationary limit. This is the principal reason why one cannot avoid solving the master equation (23) with both first and second order processes included.

To conclude, Eq. (23) captures the delicate interplay of resonant (SET) and off-resonant (COT) processes leading to mirages (COSET). The appearance of such mirages indicates that intrinsic relaxation rates are smaller than SET transport rates $(\propto \Gamma)$. "Nonequilibrium" COT is also included in this approach and the appearance of its additional features in our experiment signals a molecular device with even lower intrinsic relaxation rates, i.e., smaller than the COT relaxation rates $\left(\propto \Gamma^{2}\right)$.

\section{Breaking the rules of transport spectroscopy}

The above account of the basic rules of transport spectroscopy, although extensive, is by no means exhaustive. The key conditions are Eqs. (2) and (10), determining the resonance positions as a function of applied voltages. Readers interested mostly in the application of these rules to a high-resolution transport experiment can skip the remainder of this section and proceed directly to Sec. III. Here, we give an overview of a variety of additional effects that bend or break these rules, found in experimental and theoretical studies. In Fig. 8, we sketch a number of transport spectra that cannot be understood from what we have learned in the previous discussion. 
a

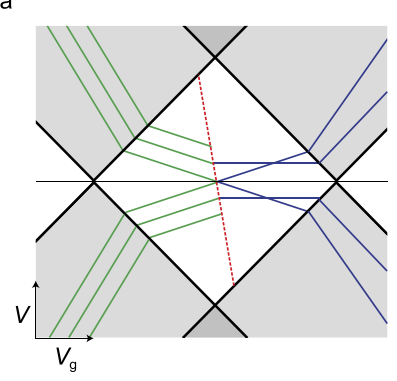

c

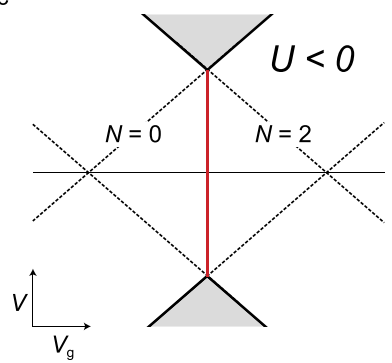

b

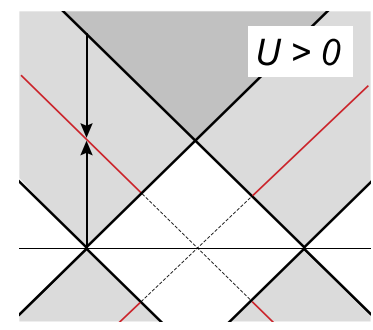

d

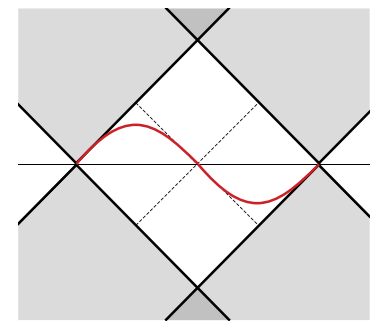

FIG. 8. Breaking the rules of transport spectroscopy. (a) Effects of nonuniform gate and bias dependence sketched after Fig. 3 of Ref. 201. Split singlet-to-triplet inelastic COT excitations (green) are tuned to degeneracy by a strong $V_{\mathrm{g}}$-dependence. Upon crossing the red line, the ground state changes from singlet to triplet, see main text. ((b) and (c)) Electron-pair tunneling resonance (red) for (b) repulsive electron interaction $U>0$ and (c) effectively attractive interaction $U<0$. (d) Transport feature (red) due to coherent spin-dynamics on a single, interacting orbital coupled to nearly antiparallel ferromagnets. Although it looks like a resonance with anomalous gate and bias dependence, it does not correspond to any state on the system. It is instead a sharp amplitude modulation caused by the orientation of the accumulated nonequilibrium spin relative to the electrode polarization vectors.

\section{Nonuniform level shifts due to voltages}

The assumption made so far [Sec. II A 1] that all energy levels are uniformly shifted by applied gate and bias voltages may not be valid in the case of local electric field gradients. In fact, this was already seen in the first experiment on gated COT spectroscopy of a single-triplet semiconductor $\operatorname{dot}^{130}$ due to the change of the confining potential with gate voltage. In molecular junctions, this has also been observed. Figure 8(a) schematizes how the transport spectrum in Ref. 201 displays such effects. In this case, the COT resonances can still be identified as weakly gate-dependent resonances, which is not a trivial issue as the experiments in Ref. 202 show. However, a qualitatively new and strongly gate-dependent resonance $^{201,203}$ (red line) appears upon ground state change. Piecing together all the evidence, it was shown that this effect originates from a change in amplitude of the COT background, without requiring the introduction of any additional states into the model. These effects are included in Eq. (23), which was shown ${ }^{203}$ to reproduce the experimental data of Ref. 201 in detail.

\section{Pair tunneling}

In all the schematics so far, we left out resonances that are caused by electron pair tunneling. These are described ${ }^{70,204}$ by the rates $\mathbf{W}^{2,0}$ and $\mathbf{W}^{0,2}$ included in the master equation (23). In Fig. 8(b), we sketch where these pair-tunneling resonances (red lines) are expected to appear: their positions are obtained by taking the bias-averaged positions of the two subsequent SET resonances. This condition follows by requiring the maximal

energy of an electron pair in the electrode $r$ to match a corresponding molecular energy change. For example, for a single orbital at energy $\epsilon$, one obtains $2 \mu_{r}=E_{g}^{N+2}-E_{g}^{N}=2 \epsilon+u$ where $u$ is the charging energy. This gives a bias window in which pair tunneling $N \leftrightarrow N+2$ can contribute to transport,

$$
\mu_{\mathrm{L}} \geq \epsilon+\frac{1}{2} u \geq \mu_{\mathrm{R}}
$$

provided that the $N$ and/or the $N+2$ state is occupied. The effective charging energy for each electron is halved since the energy $u$ is available for both electrons together in a single process. Although small (comparable with COT), its distinct resonance position and shape clearly distinguish the pair-tunneling current from the SET current, Ref. 204, that dominates in the resonant regime where it occurs.

\section{Electron attraction}

Clearly, pair tunneling effects are expected to become important if the effective interaction energy $u$ is attractive. ${ }^{205-209}$ Such attraction in fact appears in various systems. In molecular systems this is known as electrochemical potential-inversion. ${ }^{210}$ In artificial QDs, ${ }^{213}$ a negative $u$ has been observed experimentally ${ }^{211,212}$ in transport spectra of the type sketched in Fig. 8(c), see also Ref. 205. Interestingly, in this case, the ground state has either $N$ or $N+2$ electrons and never $N+1$ since starting from $(N, g)$ the single-electron transition energies $E_{g}^{N+1}-E_{g}^{N}$ and $E_{g}^{N+2}-E_{g}^{N+1}$ are higher than the electron-pair transition energy per electron $\left(E^{N+2}-E^{N}\right) / 2$. This is also included in the approach (23), see Ref. 200.

\section{4. "Coherence" effects}

Finally, we turn to the assumption used in Sec. II A 2 that the molecular state is described by "classical" occupation probabilities of the quantum states (statistical mixture). For instance, each degenerate spin multiplet is treated as an "incoherent" mixture of different spin projections (no quantum superpositions of spin-states). Equivalently, the spin has no average polarization in the direction transverse to the quantization axis.

However, when in contact with, e.g., spin-polarized electrodes, such polarization does arise already in order $\Gamma$. In that case, one must generalize Eq. (23) to include off-diagonal density-matrix in the energy eigenbasis. (The off-diagonal elements also come into play when going to order $\Gamma^{2}$, see discussion in Sec. II B 2 b.) In physical terms, this means that one must account for the coupled dynamics of charge, spin-vector, and higher-rank spin tensors. ${ }^{214,215}$ In the SET regime, such effects can lead to a nearly $100 \%$ modulation of the transport current $^{214,216}$ due to quantum interference. This emphasises that ${ }^{217}$ SET - the first order approximation in $\Gamma$ - is not "incoherent" or "classical" as some of the nomenclature in Table I seems to imply.

Similar coherence effects can arise from orbital polarization in $\mathrm{QDs}^{217-221}$ and STM configurations, ${ }^{222}$ from an interplay between the spin and orbital coherence, ${ }^{78,223,224}$ or from charge superpositions of electron pairs. Finally, for high-spin systems, coherence effects of tensorial character can arise. This leads to the striking effect that in contact with ferromagnets (vector polarization) they can produce a magnetic anisotropy 
(tensor), ${ }^{215,225}$ see also related work. ${ }^{226-229}$ An extension of the approach (23) also describes these effects. ${ }^{215,230}$

The perhaps most striking effect of spin-coherence is depicted in Fig. 8(d): SET resonances can split for no apparent reason ${ }^{225}$ and wander off deep into the COT regime ${ }^{230}$ (red line). Depending on the junction asymmetry, this feature of coherent nonequilibrium spin dynamics can appear as a pronounced gate-voltage dependent current peak or as a feature close to the linear response regime, mimicking a Kondo resonance, see also Ref. 221.

\section{SPECTROSCOPY OF A HIGH-SPIN MOLECULE}

In the second part of this paper, we present feature-rich experimental transport spectra as a function of gate-voltage and magnetic field. Their analysis requires all the spectroscopic rules that we outlined in the first part of the paper. We show how the underlying Hamiltonian model can be reconstructed from the transport data, revealing an interesting high-spin quantum system with low intrinsic relaxation.

The molecule used to form the junction is a $\mathrm{Fe}_{4}$ singlemolecule magnet (SMM) with formula $\left[\mathrm{Fe}_{4}(\mathrm{~L}) 2(\mathrm{dpm}) 6\right]$. Et2O where Hdpm is 2,2,6,6-tetramethyl-heptan-3,5-dione. Here, H3L is the tripodal ligand 2-hydroxymethyl-2phenylpropane-1,3-diol, carrying a phenyl ring. ${ }^{231}$ After molecular quantum-dot formation, the device showed interesting isotropic high-spin behavior and the clearest signatures of COSET to date ${ }^{63,130}$ for any quantum-dot structure. The device showed no significant anisotropy splittings of spin multiplets in transport, see the discussion below. Before turning to the measurements and their analysis, we first discuss specific challenges one faces probing spin-systems using either COT or SET spectroscopy.

\section{A. Principles of spin-spectroscopy}

Isotropic, high-spin molecules have molecular states labeled by the spin length $S$ and spin-projection $M$. To detect them, two types of selection rules are frequently used in STM and QD studies. Using these, we construct the possible spectroscopic COT and SET fingerprints that we can expect to measure.

\section{Spin selection rules for COT}

Spectroscopy using COT conductance as a function of magnetic field $B$ (spin-flip spectroscopy ${ }^{18}$ ) has been a key tool in both STM and break-junction studies. This approach assumes that virtual charging processes dominate. These processes involve two electrons for which the selection rules (17) apply.

However, for high-spin molecules considered here, there can be multiple spin-spectrum assignments that fit the same COT transport spectrum. An indication for this is that in the present experiment some of the spectra are very similar to those of entirely different nanostructures. ${ }^{232}$

To see how this comes about, we construct in Figs. 9(a)9(c) the three possible different fingerprints that two spinmultiplets can leave in the COT transport spectrum based on selection rules (17) alone. For simplicity, we assume that all processes start from the ground state $(N+1, g)$, i.e., in the a

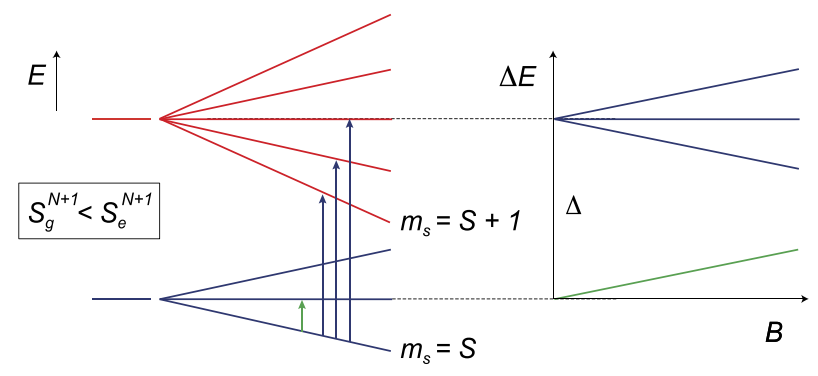

b

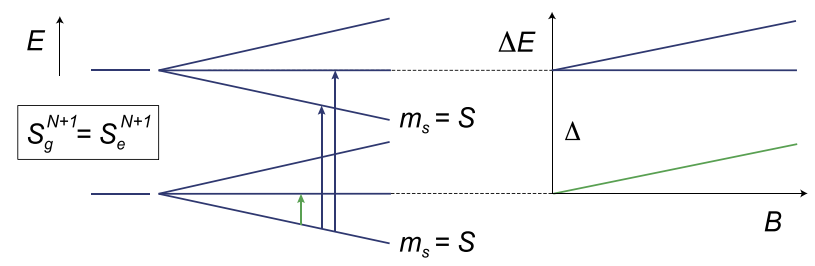

c

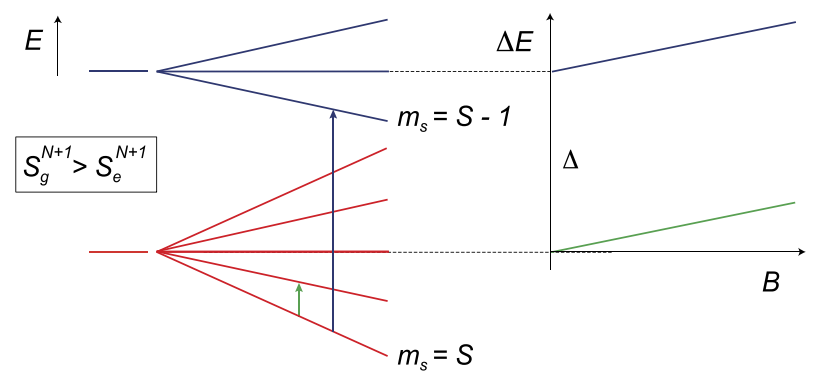

FIG. 9. COT spectroscopy of a high-spin molecule. The left panels in (a)(c) show COT transitions between energy levels $(E)$ vs. a magnetic field $B$. The right panels show the corresponding transport spectra, i.e., the resonant bias positions in $\mathrm{d} I / \mathrm{d} V$ matching an energy difference $(\Delta E)$. (a) If the spin increases upon excitation, $S_{e}^{N+1}=S_{g}^{N+1}+1$, there is a three-fold splitting of the transport-spectrum (blue) starting at $V=\Delta$ for $B=0$ due to the transitions to the excited multiplet. The ground multiplet gives a line (green) starting at $V=0$ and increasing with $B$ if $S_{g}^{N+1} \geq 1 / 2$. Only for $S_{g}^{N+1}=0$, this green line is missing. (b) If the spin length does not change upon excitation, $S_{e}^{N+1}=S_{g}^{N+1}$, the excited multiplet appears in the transport spectrum through a double line starting at $V=\Delta$. The ground multiplet gives a line (green) starting at $V=0$ and increasing with $B$ if $S_{g}^{N+1} \geq 1 / 2$. Clearly, for $S_{g}^{N+1}$ $=0=S_{e}^{N+1}$, the $B$-dependent lines are missing. (c) If the spin length decreases upon excitation, $S_{e}^{N+1}=S_{g}^{N+1}-1$, the excited multiplet appears in the transport spectrum through a single line (blue) starting at $V=\Delta$, increasing with $B$. Since in this case the ground spin $S_{g}^{N+1}$ is always nonzero, there is an intramultiplet line (green) starting at $V=0$.

"equilibrium" COT approximation discussed in Sec. II B 1. This figure shows that one can determine only whether the spin value changes by 1 or remains the same upon excitation, but not on the absolute values of the spin lengths (unless the ground state has spin zero).

\section{Spin selection rules for SET-spin blockade}

A second key tool in the study of spin effects is the transport in the SET regime. ${ }^{36,47,49-52}$ This provides additional constraints that reduce the nonuniqueness in the COT spin-assignment.

In the SET regime, the linear-transport part is governed by the transition between the two ground-state multiplets with different charge, $(N, g)$ and $(N+1, g)$, for which selection rules (3) hold. As sketched in Fig. 10, if linear SET transport is observed, then the ground-state spin values are necessarily 

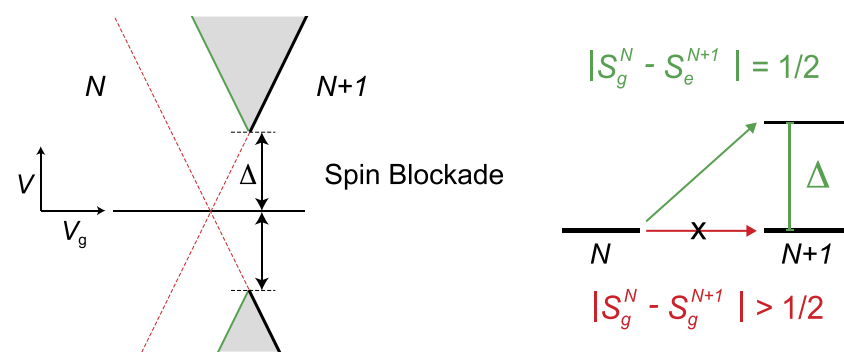

FIG. 10. SET spectroscopy of a high-spin molecule: spin blockade. If ground state transitions are spin-forbidden, $\left|S_{g}^{N+1}-S_{g}^{N}\right|>1 / 2$, then the SET transport is suppressed (red dashed cross). Transport sets in only when a finite bias makes the lowest spin-compatible excitation energetically accessible. This can be either and $N+1$ state with $\left|S_{e}^{N+1}-S_{g}^{N}\right|=1 / 2$ (shown) or an $N$ electron state with $\left|S_{g}^{N+1}-S_{e}^{N}\right|=1 / 2$ (not shown).

linked by

$$
\left|S_{g}^{N+1}-S_{g}^{N}\right|=\frac{1}{2}
$$

This constraint, used in Refs. 48, 49, and 233 restricts the set of level assignments inferred through COT spectroscopy on each of the two subsequent charge states, by fixing the relative ground state spins $S_{g}^{N}$ and $S_{g}^{N+1}$. Their absolute values remain, however, undetermined, unless one of the two happens to be zero. Arguments based on the presence of the additional spinmultiplets can then be used to motivate a definite assignment of spin values.

Molecules for which Eq. (25) fails can be identified by a clear experimental signature: the SET transport is blocked up to a finite bias as explained in Fig. 10. Such spinblockade has been well-studied experimentally ${ }^{234-236}$ and theoretically $^{237-241}$ and finds application in spin-qubits (Paulispin blockade). It has been reported also for a molecular junction. $^{233}$

Clearly, when several excited spin multiplets/charge states are involved, both the SET and COT spin-spectroscopy become more complex. However, selection rules similar to Eq. (25) also apply to excited states and thus lock the two spin spectra together. In addition, the nonequilibrium occupations of the states contribute to further restricts ${ }^{242}$ the set of possible spin-values as we will now illustrate in our experimental spectroscopic analysis.

\section{B. Molecular junction fabrication}

Molecular junctions are produced starting from a threeterminal solid-state device ${ }^{243,244}$ consisting of an oxide-coated metallic local gate electrode with a thin gold nanowire deposited on top. On such a device, a low-concentration solution of molecules $(\sim 0.1 \mathrm{mM})$ is drop-casted. The nanowire is then electromigrated at room temperature and allowed to selfbreak $^{243}$ so that a clean nanogap is formed, with a width of $\approx 1.5 \mathrm{~nm}$. The solution is evaporated and the electromigrated junctions are cooled down in a dilution fridge $\left(T_{\text {base }} \approx 70 \mathrm{mK}\right)$ equipped with a vector magnet and low-noise electronics. All the measurements are performed in a two-probe scheme either by applying a DC bias $V$ and recording the current $I$ or by measuring $d I / d V$ with a standard lock-in AC modulation of the bias.

A molecular junction as sketched in Fig. 1(b) is formed when a molecule physisorbs ${ }^{245}$ on the gold leads and thus establishes a tunneling-mediated electrical contact. The presence of the molecule in the junction is signaled by large SET transport gaps $U$ exceeding $100 \mathrm{meV}$ and low-bias inelastic COT fingerprints. Numerous molecular systems have been investigated in this configuration. ${ }^{13,35,47-51,82,201,232,233,246-251}$

As a side remark, the fact that we do not observe pronounced magnetic anisotropy effects is not unexpected: the formation of a molecular junction may involve surface interactions. In several cases, previously studied clear spectroscopic signatures of the bare molecular structure (before junction formation), such as the magnetic anisotropy, $35,48,249$ were observed also in junctions. However, depending on the mechanical and electrical robustness of the molecule, this and other spin-related parameters may undergo quantitative $^{23,51,188,252}$ or qualitative changes ${ }^{26,232}$ and sometimes offer interesting opportunities for molecular spin control. ${ }^{253}$ Image-charge stabilization effects, for example, can lead to an entirely new spin structure such as a singlet-triplet pair ${ }^{143,233}$ on opposite sides of a molecular bridge.

\section{Characterization of spin states in adjacent redox states}

We now turn to the analysis of the feature-rich transport spectrum anticipated in Fig. 1(c) and reproduced in Fig. 11. It consists of two off-resonant regimes on the left and right with fixed charge states - provisionally labeled $N$ and $N+1$ - and a resonant regime in the center surrounded by a significant crossover regime.

\section{Off-resonant analysis}

We first separately identify the electronic spectrum for each of the two accessible charge states $N$ and $N+1$ using the off-resonant approach discussed in Sec. II B.

In Fig. 11(a), we show the $\mathrm{d}^{2} \mathrm{I} / \mathrm{d} V^{2}$ color map and the corresponding $\mathrm{d} I / \mathrm{d} V$ steps for fixed $V_{\mathrm{g}}=-1.25 \mathrm{~V}$ as a function of magnetic field, $B$. Two steps (peaks in $\mathrm{d}^{2} \mathrm{I} / \mathrm{d} V^{2}$ ) starting from $V \approx 0 \mathrm{meV}$ and $V=0.78 \mathrm{meV}$ at $B=0 \mathrm{~T}$ shift upward in energy and parallel to each other as the magnetic field increases. In the standard COT picture, each step signals to the opening of an inelastic transport channel through the molecule. Transport takes place via virtual charging involving a real spinflip excitation with selection rules on spin-length $\Delta S=0,1$ and magnetization $\Delta M=0, \pm 1$. The charge of the molecule remains fixed and is labeled $N+1$. The shift in magnetic field of both steps indicates a nonzero spin ground state multiplet with spin $S_{g}^{N+1}$. According to Sec. III A, the presence of only one other finite-bias excitation shifting in the magnetic field relates the spin-values as $S_{g}^{N+1}=S_{e}^{N+1}+1$ but leaves their absolute values undetermined.

As we will see later, other spectroscopic information constrains the ground spin to be a triplet $T, S_{g}^{N+1}=1$, with a singlet excited state labeled $S$. From the COT excitation voltage, a ferromagnetic (FM) interaction energy $J=0.78 \mathrm{meV}$ can be extracted. Such type of excitation has been seen in other molecular structures. ${ }^{232,233,254,255}$ Spectra of this kind have also been obtained earlier in other quantum-dot heterostructures, such as few-electron single and double quantum dots, albeit typically characterized by smaller and antiferromagnetic couplings. ${ }^{130,256}$ 


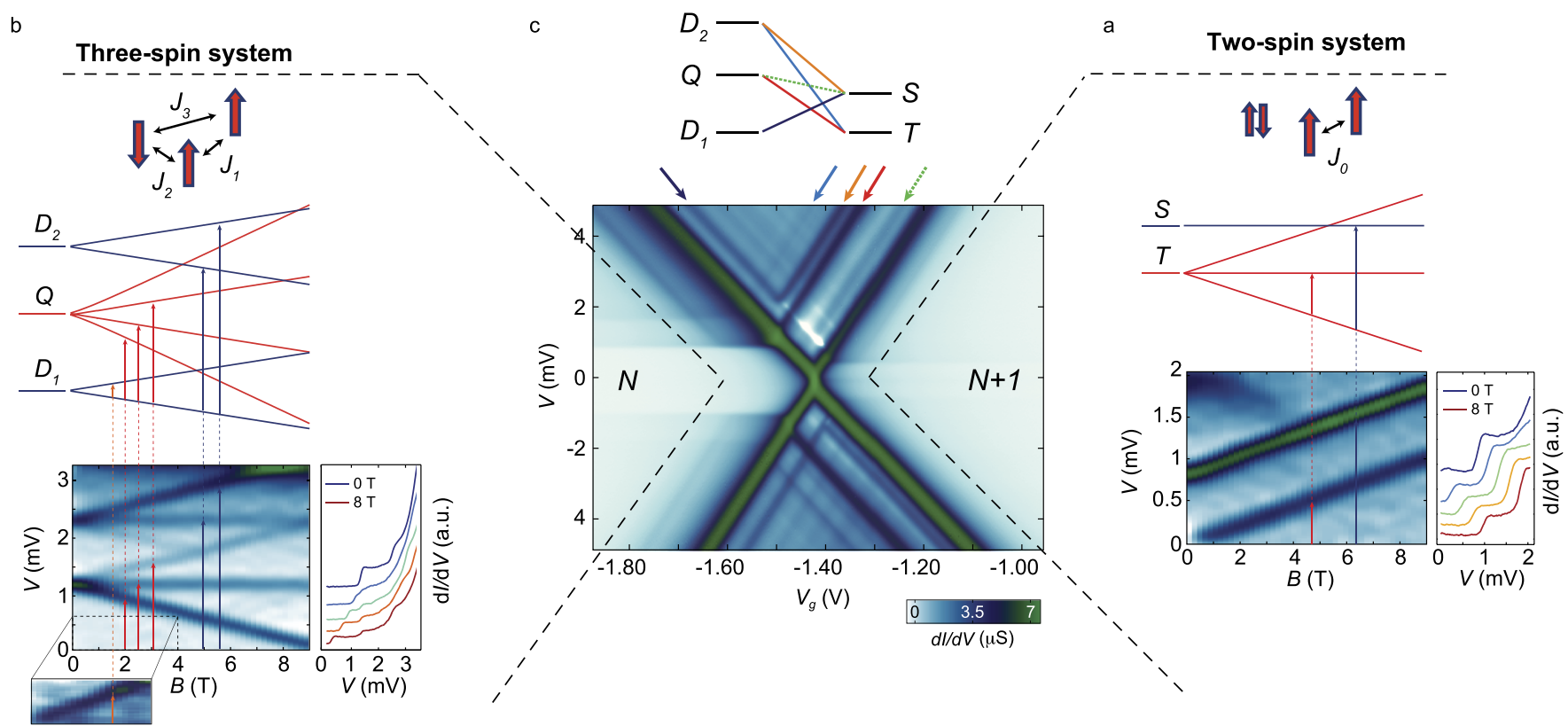

FIG. 11. The characteristics regimes of a complex molecular spin system. $\mathrm{d} I / \mathrm{d} V$ color map (stability diagram) of a high-spin molecular dot. The gate electrode allows to electrostatically vary the dot's chemical potential. This scanning in energy space grants access to real charging (mixed-valence, middle) as well as the virtual cotunneling transport regimes (far left and right sides, fixed charge). Between the two regimes, the hybrid COSET regime is visible where excitation (relaxation) is dominated by COT (SET). COT spectroscopy at $V_{\mathrm{g}}=-1.81 \mathrm{~V}$, in (b), $\left(V_{\mathrm{g}}=-1.25 \mathrm{~V}\right.$, in (a)) in the magnetic field reveals the presence of a three (two) spin system with specific ferro-/antiferromagnetic exchange couplings. The color-coded arrows indicate the transitions between the different spin multiplets of the three- and two-spin systems.

We now change the gate voltage to more negative values so that the molecule is oxidized $N+1 \rightarrow N$, i.e., we extract exactly one electron from the molecule. This can be inferred from the SET transport regime that we traverse along the way. In this new charge state, we perform an independent off-resonant spectroscopy. In Fig. 11(b), we show $\mathrm{d}^{2} I / \mathrm{d} V^{2}$ for $V_{\mathrm{g}}=-1.81 \mathrm{~V}$ as a function of the magnetic field $B$ with corresponding $\mathrm{d} I / \mathrm{d} V$ line cuts. At $B=0 \mathrm{~T}$, two sets of peaks in $\mathrm{d}^{2} I / \mathrm{d} V^{2}$ appear at $V=1.2 \mathrm{meV}$ and $V=2.26 \mathrm{meV}$ and split each in three peaks at higher magnetic fields. A weak excitation shifting upwards in $B$ from $V=0 \mathrm{~V}$ is also present. With the help of Fig. 9(a), the weak excitation and the first set of peaks are associated with $S_{e}^{N}=S_{g}^{N}+1$, while the second set, corresponding instead to the spectrum depicted in Fig. 9(b), fixes the spin to $S_{e^{\prime}}^{N}=S_{g}^{N}$. The crucial information provided by the clear absence of spin blockade in the intermediate SET regime eventually constrains $S_{g}^{N}$ to $1 / 2$ or $3 / 2$ according to (25). The only two spin configurations compatible with the observations are therefore: a ground doublet $D_{1}$, an excited quartet $Q$, and a second doublet $D_{2}$ or, alternatively, a ground quartet, an excited sextuplet, and a quartet. As we will see in Sec. III C 3, the latter can be rigorously ruled out by analyzing the SET spectrum.

The presence of the excited quartet state $Q$ implies that the charge state $N$ is a three-spin system, $N=3$, as sketched in the top panel of Fig. 11(b). The system with one extra electron in Fig. 11(a) is thus actually a $N+1=4$ electron system with one closed shell, as sketched in the figure. Upon extraction of an electron, the spectrum of the molecular device changes drastically, transforming from a ferromagnetic highlow spin spectrum for $N+1=4$ into a nonmonotonic low-highlow spin excitation sequence for $N=3$. The spin-excitation energies extracted from the two independent COT analyses are

$$
\left\{\begin{array}{l}
E_{Q}-E_{D_{1}}=1.2 \mathrm{meV} \\
E_{D_{2}}-E_{D_{1}}=2.26 \mathrm{meV}
\end{array} \text { for } \quad N=3\right.
$$

and

$$
E_{S}-E_{T}=0.78 \mathrm{meV} \text { for } N+1=4 .
$$

These energy differences provide the starting point of a more atomistic modeling of the magnetic exchanges in the two charge states. We stress that for the transport spectroscopy this is not necessary and it goes beyond the present scope. We only note that while the $N+1=4$ state requires only one fixed ferromagnetic exchange coupling $J_{0}=E_{S}-E_{T}$ [Fig. 11(a)] together with the assumption that two other electrons occupy a closed shell; the $N=3$ spectrum requires, in the most general case, three distinct exchange couplings between the three magnetic centers [Fig. 11(b)]. These relate to the two available energy differences through $E_{Q}-E_{D_{1}}$ $=\left(J_{1}+J_{2}+J_{3}\right) / 2+X / 2$ and $E_{D_{2}}-E_{D_{1}}=X$ to a complicated function $X\left(J_{1}, J_{2}, J_{3}\right)$. Since this involves three unknowns for two splittings, only microscopic symmetry considerations or detailed consideration of the transport current magnitude are needed to uniquely determine the microscopic spin structure.

This type of microscopic modeling has proven successful in many instances, see Ref. 25 and the references therein. However, the underlying assumptions on localized spins and fixed charge occupations can only be made when sufficiently far away from resonance, i.e., such that COSET does not take place as expressed by conditions (21) and (22). 


\section{Resonant analysis}

Using the ability to control the energy levels with the gate, the COT analysis can be complemented by a SET spectroscopy in the central part of Fig. 11(c). Here, real charging processes dominate. For example, starting from the ground state $D_{1}$, addition of a single electron leads to occupation of the $T$ ground state. This is evidenced by the clear presence of a SET regime of transport down to the linear-response limit. Inside the resonant regime, additional lines parallel to the edges of the cross appear as well. As we explained in Fig. 3, these correspond to real charging processes where excess (deficit) energy is used to excite (relax) the molecule. These additional lines, schematized for our experiment in Fig. 12(a), fall into two categories according to the following criteria:

(a) Lines terminating at the boundary of the SET regime correspond to the ground $N$ to excited $N \pm 1$ transitions or vice versa.

(b) Lines that never reach the SET boundary but terminate inside the SET regime at a line parallel to this boundary. These correspond to excited $N$ to excited $N \pm 1$ transitions. Their earlier termination indicates that that the initial excited state must become first occupied through another process. The line at which it terminates corresponds to the onset of this activating process.

In Figs. 11(c) and 12(a), the SET transitions $D_{1} \leftrightarrow S$, $Q \leftrightarrow T$, and $D_{2} \leftrightarrow T$ fall into category (a), while the $D_{2} \leftrightarrow S$ and $Q \leftrightarrow S$ transitions belong to (b). Due to the large difference in spin-length values of the spin-spectra, the latter transition, a

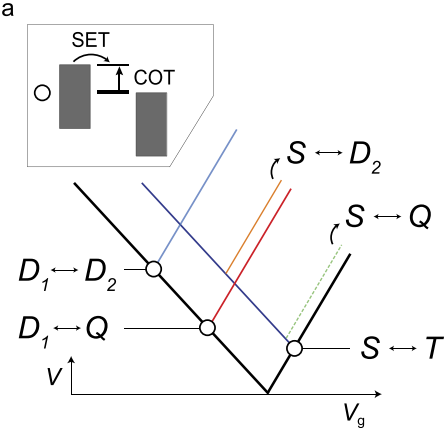

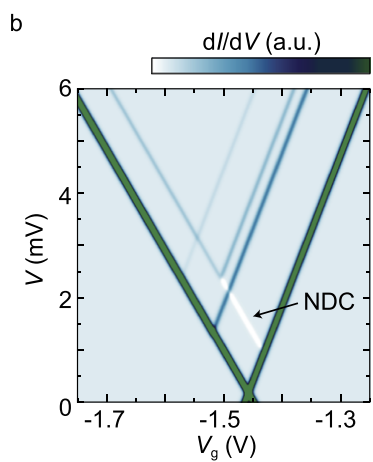

FIG. 12. Connecting the off-resonant and resonant analyses. (a) Schematic of COT and SET excitations observed in Fig. 11. SET transitions between a ground and excited state (blue, red) reach the boundary of the resonant regime at the black circle from where they continue horizontally as a COT excitation. The inset depicts the chemical potential configuration at such a black circle where COT and SET connect. The SET transitions between two excited states (orange, green) do not connect to any COT excitation. (b) SET transport spectrum computed using the master equations (7)-(9). The energies are extracted independently from the two COT spectra in Fig. 11, and the capacitive parameters $\alpha_{\mathrm{g}}=0.012, \alpha_{\mathrm{L}}=\alpha_{\mathrm{g}} / 0.6$, and $\alpha_{\mathrm{R}}=\alpha_{\mathrm{g}} / 0.4$ are fixed by the observed slopes of the SET lines [cf. Fig. 3(a)], leaving the tunnel rates (28) and (29) as adjustable parameters. The broadening of the $\mathrm{d} I / \mathrm{d} V$ peaks in the experiment is due to tunneling, $\Gamma \approx 4.6 \mathrm{~K} \sim 0.4 \mathrm{meV}$ (FWHM), rather than temperature, $T \approx 70 \mathrm{mK} \sim 6 \mu \mathrm{eV}$. Eqs. (7)-(9) do not include this $\Gamma$-broadening and we crudely simulate it by an effective higher temperature $T^{*}=270 \mathrm{mK} \sim 23 \mu \mathrm{eV}$. The master equations (7)-(9) are valid for small effective tunnel coupling $\Gamma^{*} \ll T^{*}$, which only sets the overall scale of plotted SET current and not the relative intensities of interest. The caption to Fig. 16 explains that $\Gamma^{*}$ should not be adjusted to match the larger experimental current magnitude. marked in dashed-green, is actually forbidden by the selection rules (3). Following this line, we find that it terminates at a strong negative differential conductance (NDC) feature (white in the stability diagram in Fig. 11) marking the onset of the transition $D_{1} \leftrightarrow S$.

To test our earlier level assignment based COT, we now compute the expected SET transport spectrum the first-order ( $\Gamma)$ master equations (7)-(9) and by adjusting the result, we extract quantitative information about the tunnel coupling. The model Hamiltonian is constructed from the energies (26)(27) and their observed spin-degeneracies. Assuming that spin is conserved in the tunneling, the rates between magnetic sublevels are fixed by Clebsch-Gordan spin-coupling coefficients ${ }^{176,241}$ incorporating both the SET and COT selection rules, Eqs. (3) and (17). The tunnel parameters in units of an overall scale $\Gamma^{*}$ are adjusted to fit the relative experimental intensities,

$$
\left\{\begin{array}{l}
\Gamma_{D_{1}, T}=1.0 \Gamma^{*} \\
\Gamma_{Q, T}=1.0 \Gamma^{*} \\
\Gamma_{D_{2}, T}=0.25 \Gamma^{*} \quad \text { (weak intensity) }
\end{array}\right.
$$

and

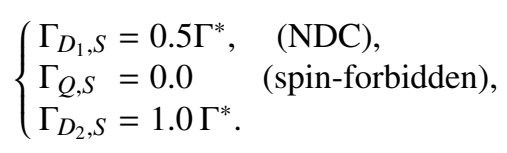

Their relative magnitudes provide further input the further microscopic modeling of the 3-4 spin system mentioned at the end of Sec. III C 1. As shown in Fig. 12(b), the resonant (SET) part of the experimental conductance in Fig. 11(c), as schematized in Fig. 12(a), is reproduced in detail. This includes transitions exciting the molecule from its ground states, but also a transition between excited states. ${ }^{257}$ The NDC effect is explained in more detail later on together with the full calculation in Fig. 15.

\section{Connecting the off-resonant and resonant analyses}

As discussed in Figs. 4 and 5 and indicated in Fig. 12(a), the SET excitations corresponding to the ground $N$ to excited $N \pm 1$ transitions connect continuously to the COT excitations. Those corresponding to two excited states, each of the different charge state, have no corresponding COT excitation to connect to. In this sense, the SET spectrum effectively ties the two separately obtained COT spin spectra and allows a consistency check on their respective level assignments, cf. Sec. II C 2.

For instance, from the fact that the $Q \leftrightarrow T$ transition is clearly visible-marked red in Fig. 11(c)—we conclude that the first excited multiplet of the $N$ charge state cannot be a sextuplet $(S=5 / 2)$ since such SET transition would be spinforbidden and thus weak. Another example is given by the presence of the $S \leftrightarrow D_{2}$ SET transition [orange in Fig. 11(c)], which implies that the second excited multiplet of the $N$ charge state cannot be a quartet. The fact that this transition does not continue into any of the COT ones is also consistent with its excited-to-excited character.

These two exclusion considerations were anticipated in Sec. III C 1 and are crucial for our off-resonant assignment in the three-electron state and has now allowed us to 
reverse-engineer the effective many-electron molecular Hamiltonian. With this in hand, we turn to the main experimental findings and investigate the "nonequilibrium" COT through the molecule [Sec. III D] and the crossover regime where real and virtual tunnelings nontrivially compete in the relaxation of spin excitations [Sec. III E].

\section{Pump-probe spin spectroscopy by nonequilibrium electron COT}

We first investigate how COT spectrum evolves as we further approach the SET regime from either side. Fig. 13(a) shows the analogous of Fig. 11(a) but closer to the SET regime, at $V_{\mathrm{g}}=-1.32 \mathrm{~V}$. A horizontal, $B$-field independent line appears (dotted green line in the center-panel schematic) that terminates at $B_{c} \approx 4.5 \mathrm{~T}$, precisely upon crossing the intra-triplet excitation (blue line). This indicates that the excited triplet ( $\operatorname{spin} S=1$ perpendicular to the field, $M=0$ ) lives long enough for a secondary COT process to excite the system to the singlet state (reducing the spin length to $S=0$ ). Strong evidence for this is the termination of this line: once the initial state ( $M=0$ excited triplet) for this transition is no longer accessible for $B>B_{c}$, the "nonequilibrium" cascade of transitions is interrupted.

We consistently observe this effect, also when approaching the SET regime from the side of the other charge state $(N=3)$ with different spin. In Fig. 13(b), we show the magnetic

a
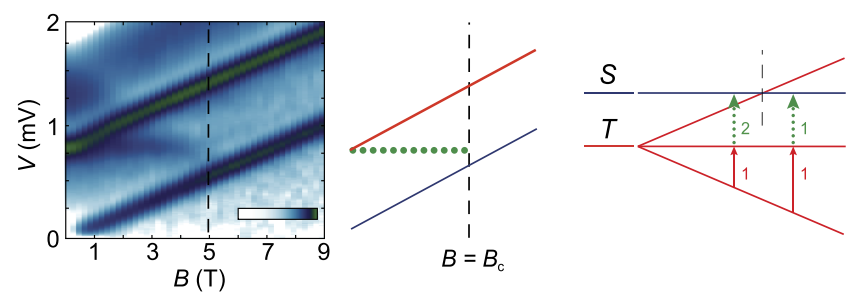

b

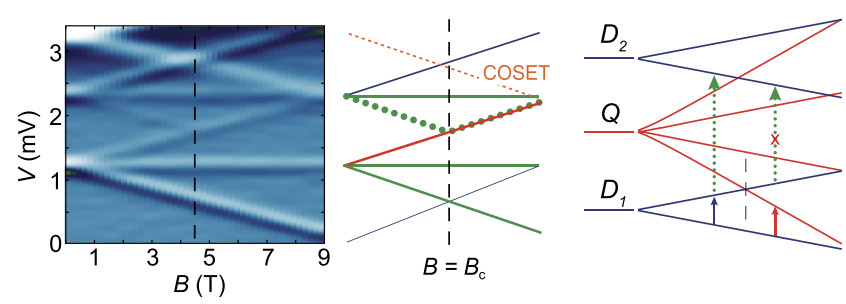

FIG. 13. Nonequilibrium spin pumping and locking mechanism. In contrast to Fig. 11, we highlight here the transitions that are involved in the spin pumping process (green dotted lines). (a) $\mathrm{d}^{2} I / \mathrm{d} V 2$ spectra measured as a function of $B$-field at $V_{\mathrm{g}}=-1.32 \mathrm{~V}$ in the $N+1=4$ charge state. The $T \leftrightarrow S$ nonequilibrium spin-excitation shows up as a weak, field-independent step vanishing at higher field. For $B<B_{c}$, the intra-triplet transition (red arrow) requires lower energy than the "nonequilibrium" $T \leftrightarrow S$ transition. For $B>B_{c}$, the intra-triplet is unlocked (activated) at an energy higher than $T \leftrightarrow S$ and only one transition of the cascade is visible. (b) $\mathrm{d}^{2} I 1 / \mathrm{d} V 2$ spectra measured as a function of $B$-field at $V_{\mathrm{g}}=-1.76 \mathrm{~V}$ in the $N=3$ charge state. Here the nonequilibrium excitation has a negative slope. For $B<B_{c}$, the excited state of the ground-state doublet $D_{1}$ is populated enough to promote a second, nonequilibrium excitation to the excited doublet $D_{2}$ (green dotted line). As $B>B_{c}$, the $D_{1} \leftrightarrow Q$ transition crosses over, lowering, in consequence, the population of the spin-up state. This results into a quench of the nonequilibrium excitation. Due to the proximity to SET regime as compared to Fig. 11(a), a COSET feature (orange dotted line) appears as a mirage of a spin-excitation. field spectrum taken at $V_{\mathrm{g}}=-1.75 \mathrm{~V}$. Here the lowest $D_{2}$ excitation gains strength ${ }^{258}$ relative to Fig. 11(b). In this case, the excited $D_{1}$ state is the starting point of a "nonequilibrium" cascade. As for the previous case, it terminates when levels cross at $B \approx 4 \mathrm{~T}$ for similar reasons: Once the $Q$ state gains occupation for $B>B_{c}$ (since the $D_{1} \leftrightarrow Q$ transition becomes energetically more favorable), the excited $M$-substates of the $D_{1}$ multiplet are depleted causing the line to terminate. In both charge states, the observed "nonequilibrium" COT current gives an estimate for the spin-relaxation time, $\tau_{\text {rel }} \gtrsim 10^{-9} \mathrm{~s}$ : to have any such pump-probe current, spin-relaxation after a pump process should not be complete before the probe process starts. The inverse of this measured current (in electrons/second) gives the above estimate.

Nonequilibrium transitions can thus give rise to clear excitations at lower energy than expected from the simple selection-rule plus equilibrium arguments of Sec. III A. In this type of process, two COT events $\left(\propto \Gamma^{2}\right)$ happen in sequence, so that a total of four electrons are involved. ${ }^{259}$ In this sense, the phenomena can be regarded as a single-molecule electronic pump-probe experiment, that is, the excess energy left behind by the first process (pump) allows the second process to reach states (probe) that would be otherwise inaccessible at the considered bias voltage. This has been successfully applied in STM studies ${ }^{120,253}$ for dynamical spin-control.

\section{E. Mirages of spin transitions "far from resonance"}

\section{Mirages}

We now further reduce the distance to resonance, again coming from either side, and enter the crossover regime discussed in Sec. II C. We are, however, still "far from resonance" by the linear-response condition (5).

In the upper panel of Fig. 14(a), we show $\mathrm{d} I / \mathrm{d} V$ traces taken at various magnetic fields for a constant gate voltage $V_{\mathrm{g}}=-1.72 \mathrm{~V}$. At high bias voltage, the $\mathrm{d} I / \mathrm{d} V$ steeply rises due to the onset of the main SET resonance. Below this onset, we note a step-like excitation at $V=2.1 \mathrm{meV}$ (black arrow) which shifts up in the magnetic field with the same $g$-factor $(\approx 2)$ as the other lower-lying COT excitations. ${ }^{260}$ If one adopts the off-resonant picture this excitation is attributed to the opening of an independent COT channel. This attribution proves to be erroneous: Keeping $B=3$ T fixed and varying the gate voltage (Fig. 14(a), lower panel), we observe that the lower excitations are left unchanged, whereas the higher one under consideration shifts linearly with $V_{\mathrm{g}}$, revealing that it is not a COT excitation.

This attribution to COT can be further ruled out by looking at the full gate-voltage dependence in the stability diagram shown in the left panel of Fig. 14(b). The excitation (red arrow) has the same gate dependence as the SET resonances, even though it is definitely not in the resonant regime by the linearresponse criterion (5). In fact, it is a COSET mirage of the same lowest gate-voltage independent COT excitation as we explained in Fig. 6. Its bias (energy) position does not provide information about the excitation energy $\Delta$ : depending on the energy level position, the mirage's excitation voltage $V^{*}$ can lie anywhere above the COT threshold voltage $V=\Delta$, see Sec. II C. 

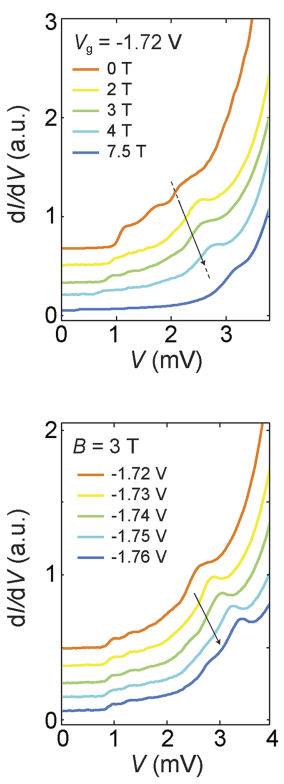
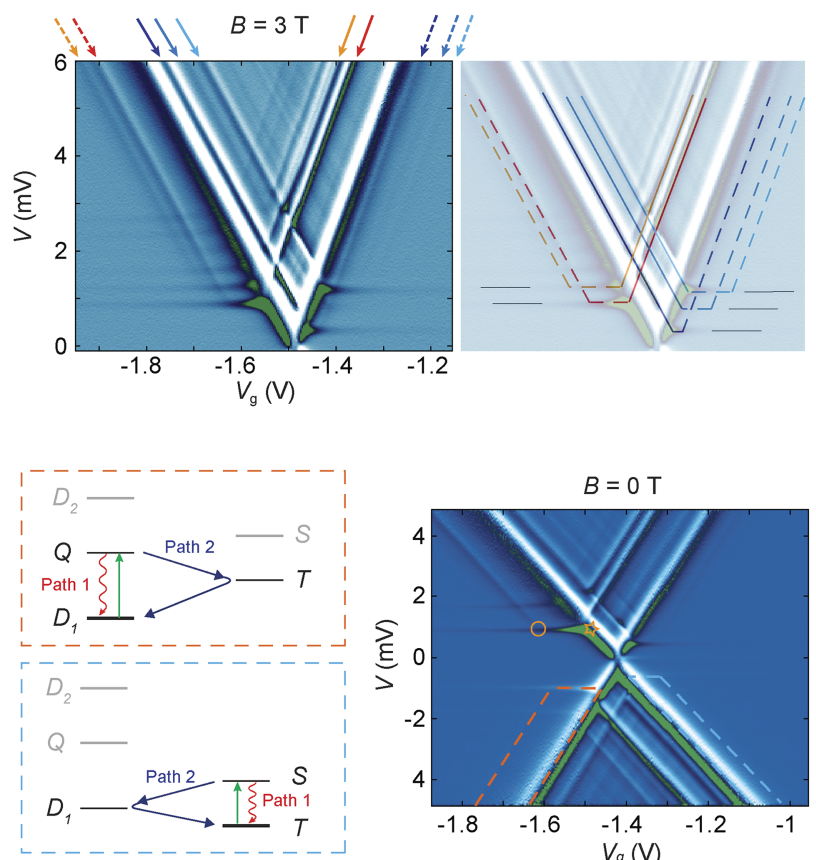
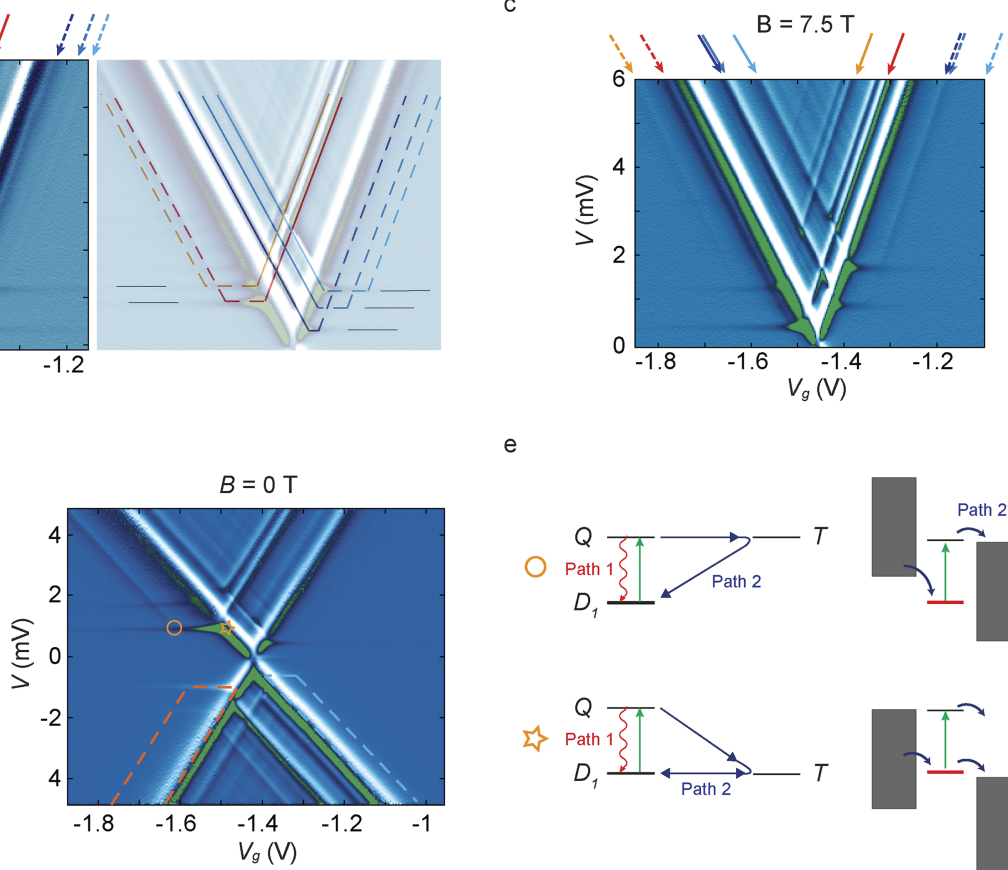
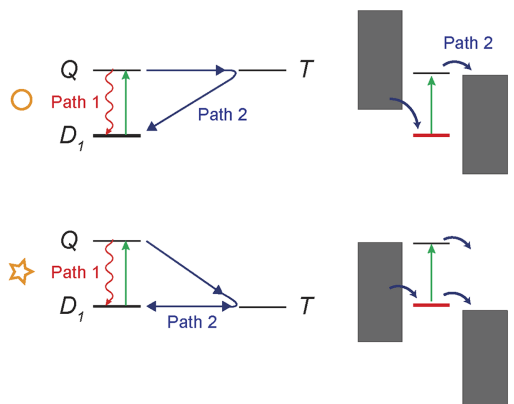

FIG. 14. Transport characteristics of spin COSET mirages. (a) $\mathrm{d} I / \mathrm{d} V$ spectra taken at $V_{\mathrm{g}}=-1.72 \mathrm{~V}$ for different $B$-fields (upper) and at $B=3 \mathrm{~T}$ for different values of $V_{\mathrm{g}}$ (lower). The step indicated by the black arrow Zeeman-splits as a regular magnetic transition. The same step moves to higher energies as a function of gate voltage and fixed magnetic field. (b) $\mathrm{d}^{2} I / \mathrm{d} V^{2}$ color map at $B=3 \mathrm{~T}$. Red (blue) arrows and lines indicate the SET excitations that extend their mirror images into the COT regions of the $N(N+1)$ charge state. (c) $\mathrm{d}^{2} I / \mathrm{d} V 2$ color map at $B=7.5 \mathrm{~T}$. The $B$-field evolution of the mirror lines follows that of their real counterparts. (d) $\mathrm{d}^{2} I / \mathrm{d} V^{2}$ of the plot in Fig. 11(c). The cotunneling-assisted SET transport bands are highlighted. As illustrated in the schematics, mirror images are created when two or more relaxation paths compete. One path (red) involves the intra-molecular relaxation rate characteristic of the COT regime. The second, alternative path (blue) requires a charge/spin fluctuation to the $\left(N \pm 1, S_{N \pm 1}\right)$ state. (e) Energy and chemical potential schematics at the boundaries of the COSET bands. SET and COT transport occur $(\star)$. More negative gate voltages shift the $T$ state higher in energy, forbidding SET and leaving only COSET and COT competing. At the position indicated by $\circ$ further gating finally quenches COSET.

In the stability diagram in the right panel of Fig. 14(b), we connect by dashed lines all the COSET resonances to their corresponding SET excitations according to the scheme in Fig. 6. We find that mirages appear for virtually all spin-related excitations of the molecule. The stability diagram in Fig. 14(c) [same color coding as in (b)] shows that at high magnetic field $B=7.5 \mathrm{~T}$ these mirages persist.

The clearly visible COSET resonances mark the lines where the relaxation mechanism changes from virtual (COT) to real (SET) charging. They indicate that any intrinsic relaxation is comparable or slower than SET. (If the intrinsic relaxation was much faster, compared to SET, it would dominate everywhere, giving a much smaller change in the current at COSET resonances.) Mirages are thus a signature of slow intramolecular relaxation; in particular, they indicate that the intrinsic relaxation time is bounded from below by the magnitude of the observed SET currents $\tau_{\text {rel }} \gtrsim \Gamma^{-1} \sim 10^{-11} \mathrm{~s}$ using the same estimation procedure as above. This is consistent with the sharper lower bound we obtained above from nonequilibrium COT spectroscopy.

\section{Spin relaxation}

To shed light on what the relaxation mechanism by transport entails in our device, we return to the stability diagram for $B=0 \mathrm{~T}$, which is shown as $\mathrm{d}^{2} I / \mathrm{d} V^{2}$ in the right panel of Fig. 14(d). Highlighted at negative bias are the two crossover-regime bands within which COSET, rather than COT, dominates the relaxation. The left panel shows the different relaxation paths for these two bands.

Focusing on the orange band, we start out on the far left of Fig. 14(d) moving at fixed bias $V=1.2 \mathrm{meV}$ along the onset of inelastic COT. Fig. 14(e) depicts the corresponding energies (left) and energy differences (right). Here, the molecule is in the spin-doublet $D_{1}$ ground state and is occasionally excited to the high-spin quartet $Q$ by COT from where it relaxes via path $1\left(10^{-9} \mathrm{~s}\right)$, again by COT.

When reaching the circle (o) in Fig. 14(d), the relaxation mechanism changes: path 1 is overridden by the faster relaxation path $2\left(10^{-11} \mathrm{~s}\right)$ which becomes energetically allowed [Eq. (22)]. The top panel of Fig. 14(e) illustrates that although the ground state $D_{1}$ is off-resonant (highlighted in red), after exciting it by COT to $Q$-increasing the spin-length - the system has enough spin-exchange energy (green) to expel a single electron in a real tunneling processes leaving a charged triplet state behind.

At the star ( $\star$ ) in Fig. 14(d), the excitation mechanism changes from off-resonant to resonant, leaving the relaxation path unaltered. Now the ground state $D_{1}$ becomes unstable with respect to real charging: there is enough energy to expel an electron to the right electrode and sequentially accept another one from the left. We thus have an resonant SET transport cycle, i.e., the stationary state is a statistical mixture of the $N$ and $N+1$ ground states.

The COSET regime is delimited by mirage resonances and situated between the two positions $\circ$ and $\star$. Failure to 
a

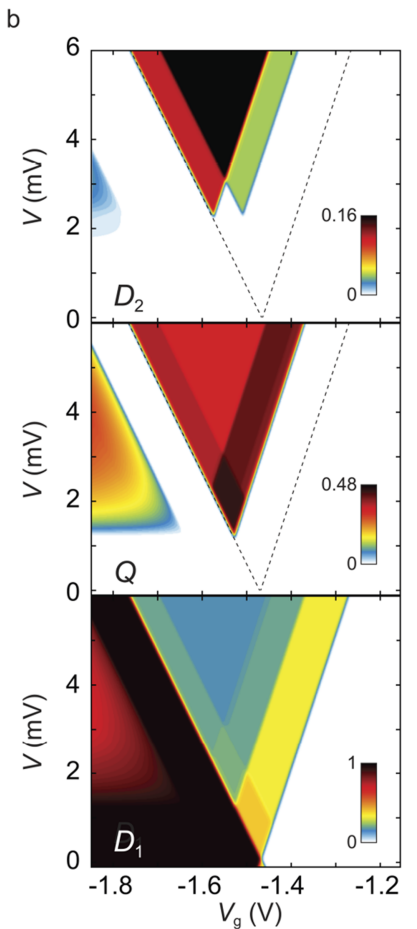

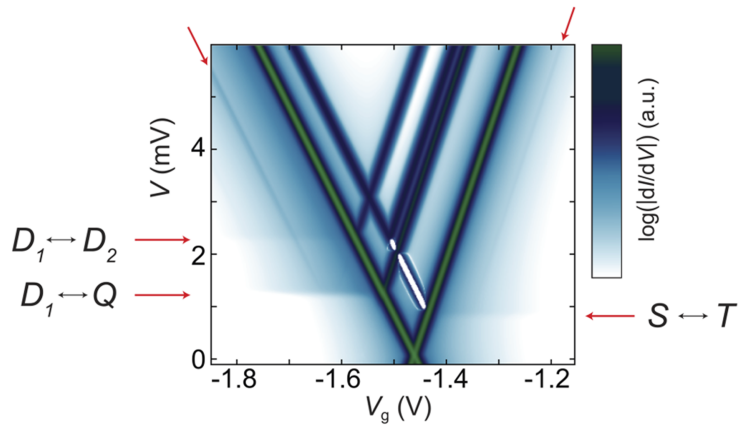

( $n+1$
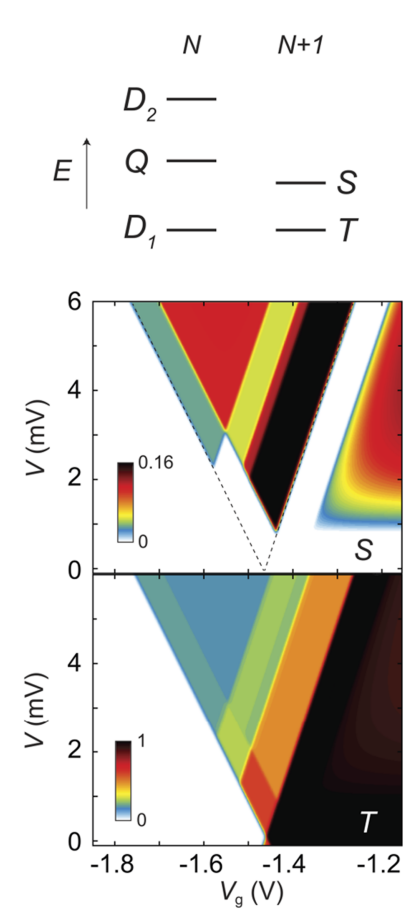

FIG. 15. Calculated stability diagram and multiplet occupations. Result obtained from the full master equation (23) and its corresponding current formula (not shown, see Refs. 70 and 192) for the same parameters as in Fig. 12(b). (a) Transport spectrum for $B=0$ corresponding to Fig. 11(c). (b) Corresponding color plots of the occupation probabilities of the five spin multiplets (probabilities summed of degenerate levels). The effective coupling $\Gamma^{*}$-merely an overall scale factor in Fig. 12(b) - now controls the magnitude of the COT and COSET current corrections relative to the SET current. Although elaborate, these corrections still neglect nonperturbative broadening effects and must kept small for consistency by explicitly setting $\Gamma^{*}=2.2 \cdot 10^{-3}, T^{*}=0.6 \mathrm{mK}=5 \cdot 10^{-5} \mathrm{meV}$. More advanced master equation approaches based on renormalization-group ${ }^{165,261}$ (RG) or hierarchical $^{262,263}$ (HQME) methods can deal with both this broadening and the corresponding larger currents.

identify the difference between this "band" and the pure COT happening on the left of $\circ$, besides yielding a wrong qualitative spin multiplet structure, leads to an overestimation of the relaxation time: in the COSET regime, the spin-excitations created by inelastic COT are quenched.

\section{Quenching of spin-excitations}

We now assess this quenching in detail for the experimental situation by a calculation based on the master equation ${ }^{70,192}$ (23) that includes all $\Gamma$ and $\Gamma^{2}$ processes using the model determined earlier [Eqs. (26)-(29)], simulating the broadening as before by an effective temperature [Fig. 12].

The computed conductance for $B=0$ is shown in Fig. 15(a). Besides the SET excitations-including the NDC effect-obtained earlier in Fig. 12(c), we capture the main features of the experimental data in Figs. 12(c) and 14(d): the three horizontal COT excitations and two prominent COSET lines.

We can now explore the nonequilibrium occupations of the five spin-multiplets as the transport spectrum is traversed. These are shown in Fig. 15(b). The lowest panels show that in the left (right) off-resonant regime the ground multiplet $D_{1}$ with $N$ electrons ( $T$ with $N+1$ electrons) is occupied with probability 1 at low bias voltage (black regions). In contrast, in the resonant regime, these two ground states are both partially occupied due to SET processes. We compare the occupations along three different vertical $\mathrm{d} I / \mathrm{d} V$ line cuts in Fig. 15(a):

(i) Increasing the bias voltage in the resonant regime, starting from $V_{\mathrm{g}}=-1.46 \mathrm{~V}$, one first encounters in Fig. 15(a) a $\mathrm{d} I / \mathrm{d} V$ dip (NDC, white). This is caused by the occupation of the $S$ state, as the $S$-panel in Fig. 15(b) shows. This drains so much probability from the $T$ multiplet [with a higher transition rate to the $D_{1}$ multiplet, Eqs. (28) and (29)] that the current goes down. Increasing the bias further depopulates the $S$ state again, thereby restoring the SET current through a series of $\mathrm{d} I / \mathrm{d} V$ peaks.

(ii) Increasing the bias voltage starting from the right offresonant regime, the excited $S$-state becomes populated by COT decreasing the average spin-length of the molecule. When crossing the COSET resonance at higher bias, this excitation is completely quenched (white diagonal band) well before reaching the resonant regime, enhances the molecular spin, restoring the triplet.

(iii) When starting from the left off-resonant regime, the population of the excited $Q$-state enhances the average spin-length of the molecule. As before, crossing the COSET resonance at higher bias quenches this excitation. Now this reduces molecular spin, restoring the doublet. Along the way, the $D_{2}$ state also becomes occupied by COT and subsequently quenched by COSET. Because of its higher energy, the white a

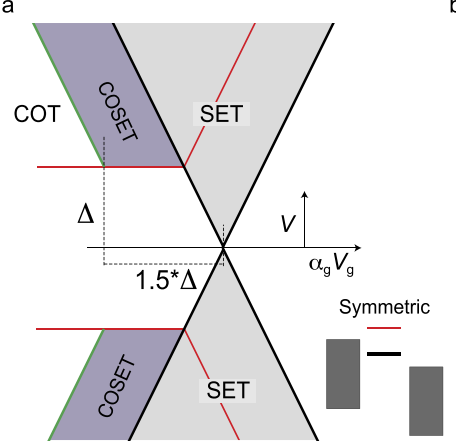

b

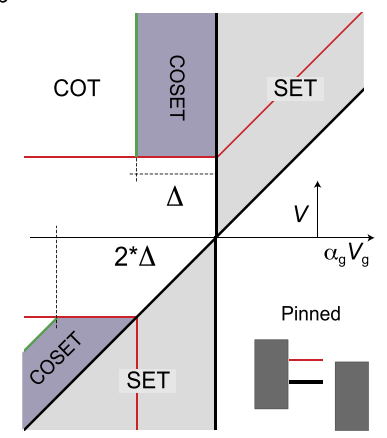

FIG. 16. How far is "off-resonant"? (a) Energy-energy stability diagram for symmetric capacitive coupling $\left(C_{\mathrm{L}}=C_{\mathrm{R}} \gg C_{\mathrm{g}}\right)$ characteristic of molecular QD devices. (b) Strongly asymmetric couplings $\left(C_{\mathrm{L}} \gg C_{\mathrm{R}}\right)$. This is typical for molecular STM junctions, where the energy levels pin to one electrode (substrate), leaving the tip electrode to act as a probe. In this case, the energy $\alpha_{\mathrm{g}} V_{\mathrm{g}}$ represents the level equationment with the Fermi-energy. 
COSET band in the $D_{2}$-panel of Fig. 15(b) is much broader.

\section{F. How far is "off-resonant"?}

The results show that the widths of the two bands where the COT excitations are quenched by COSET are unrelated to the width of the SET resonances, set by the maximum of $\Gamma$ and $T$. They are, instead, set by the excitation spectrum one wishes to probe.

In Fig. 16(a), we quantify how far the energy level has to be detuned from resonance in order avoid this quenching in our molecular QD device structure. When this detuning lies in the window $-1.5 \Delta<\alpha_{\mathrm{g}} V_{\mathrm{g}}<-0.5 \Delta$, one is sure to run into the COSET band with increasing bias. Only for $-\alpha_{\mathrm{g}} V_{\mathrm{g}}<1.5 \Delta$, there is a finite window where the excitation is not quenched. For the excitations $T, Q, D_{2}$ in our experiment, this amounts to $2.9,4.5$, and 8.5 times the SET resonance width.

In Fig. 16(b), we show the corresponding construction for strong capacitive asymmetry typical of STM setups. To avoid quenching for any bias polarity, one now needs to stay further away from resonance $-\alpha_{\mathrm{g}} V_{\mathrm{g}}<2 \Delta$. Interestingly, for $-2 \Delta<\alpha_{\mathrm{g}} V_{\mathrm{g}}<-\Delta$, the COT excitation at forward $V=\Delta$ is not quenched, whereas at reverse bias $V=-\Delta$ it is. For asymmetric junctions, the COSET mechanism thus leads to a strong biaspolarity dependence of relaxation of excitations in the nominal off-resonant regime. For $-\Delta<\alpha_{\mathrm{g}} V_{\mathrm{g}}$, one is sure to run into the COSET band for forward bias.

Whereas in the present experiment we encountered relatively low-lying spin-excitations $(\Delta \sim$ few $\mathrm{meV})$, atomic and molecular devices can boast such excitations up to tens of meV. To gauge the impact of COSET mirage resonances, consider an excitation at $\Delta \sim 25 \mathrm{meV}$ that we wish to populate by COT, e.g., for the purpose of spin-pumping. ${ }^{120,253}$ To avoid the quenching of this excitation $V=\Delta$, the distance to the Fermienergy at $V=0$ (level-equationment) needs to exceed room temperature, even when operating the device at $\mathrm{mK}$ temperatures. For vibrational and electronic excitations on the 100 $\mathrm{meV}$ scale, the implications are more severe. Moreover, even for excitations that do satisfy these constraints, cascades of "nonequilibrium" COT excitations may-if even higher excitations are available (e.g., vibrations) — provide a path to excitations that do decay by SET processes. While all these effects can be phrased loosely as "heating," in this paper we demonstrated their discrete, in situ tuneability, and the role they play as a spectroscopic tool.

\section{SUMMARY AND OUTLOOK}

We have used electron transport on a single-molecule system to comprehensively characterize the spin degree of freedom and its interaction with the tunneling electrons. Three key points-applicable to a large class of systems—emerged with particular prominence:

(i) Combining SET and COT spectroscopy in a single stable device provides new tools for determining spin properties within and across molecular redox states. This is crucially relevant for the understanding of the different spin-relaxation mechanisms, even in a single redox state. (ii) Nonequilibrium pump-probe electron excitation using two COT processes (four electrons) was demonstrated in our three-terminal molecular device and signals a substantial intrinsic spin relaxation time of about $1 \mathrm{~ns}$, much larger than the transport times.

(iii) Mirages of resonances arise from the nontrivial interplay of SET and COT. These COSET resonances signal a sharp increase of the relaxation rate and can occur far away from resonance (many times the resonance width). This limits the regime where spin-pumping works by quenching nonequilibrium populations created by a COT current.

The appearance of a mirage of a certain COT excitation indicates that the relaxation of the corresponding molecular degree of freedom dominates over all possible unwanted, intrinsic mechanism. Thus, "good" devices show mirages and "even better" devices show nonequilibrium COT transitions.

Energy level control turns out to be essential for "imaging" in energy space which allows one to distinguish mirages from real excitations. Whereas real-space imaging seems to be of little help in this respect, the mechanical gating possible with scanning probes overcomes this problem. However, even when energy-level control is available, spectroscopy of molecular junctions still requires extreme care as we illustrated in Sec. II D by several examples that break spectroscopic rules. Moreover, our work underlines that level equationment has to be treated on a more similar footing as coupling $(\Gamma)$ and temperature $(T)$ broadening in the engineering of molecular spin structures and their spin-relaxation rates. ${ }^{120,253}$

Beyond electron charge transport, recent theoretical work $^{200,264}$ has pointed out that the importance of COSET is amplified when moving to nanoscale transport of heat. ${ }^{31}$ Whereas in charge transport all electrons carry the same charge, in energy transport electrons involved in COSET processes effectively can carry a quite different energy from that acquired in a COT process only and therefore dominate energy currents. ${ }^{200,264}$ Thus, the sensitivity to spin-relaxation processes is dramatically increased in heat transport, indicating an interesting avenue ${ }^{200}$ for a spin-caloritronics ${ }^{265}$ on the nanoscale.

\section{ACKNOWLEDGMENTS}

We thank A. Cornia for the synthesis of the molecules, M. Leijnse and M. Josefsson for assistance with the calculations, and S. Lounis, M. dos Santos Dias, and T. Esat for discussions. We acknowledge financial support by the Dutch Organization for Fundamental research (NWO/FOM) and an advanced ERC grant (Mols@Mols). M.M. acknowledges financial support from the Polish Ministry of Science and Higher Education through a young scientist Fellowship (No. 0066/E-336/9/2014), and from the Polish Ministry of Science and Education as Iuventus Plus Project (No. IP2014 030973) in years 2015-2017, and from the Knut and Alice Wallenberg Foundation. E.B. thanks funds from the EU FP7 program, Project No. 618082 ACMOL through a NWO-VENI fellowship. 
${ }^{1}$ Molecular Magnets: Physics and Applications, NanoScience and Technology, edited by J. Bartolomé, F. Luis, and J. F. Fernández (Springer, Heidelberg, 2014).

${ }^{2}$ C. Huang, A. V. Rudnev, W. Hong, and T. Wandlowski, "Break junction under electrochemical gating: Testbed for single-molecule electronics," Chem. Soc. Rev. 44, 889-901 (2015).

${ }^{3}$ Handbook of Single-Molecule Electronics, edited by K. Moth-Poulsen (Pan Stanford Publishing Pte. Ltd., Singapore, 2016).

${ }^{4}$ M. L. Perrin, E. Burzurí, and H. S. J. van der Zant, "Single-molecule transistors," Chem. Soc. Rev. 44, 902-919 (2015).

${ }^{5}$ Y.-S. Chen, M.-Y. Hong, and G. S. Huang, "A protein transistor made of an antibody molecule and two gold nanoparticles," Nat. Nanotechnol. 7, 197-203 (2012).

${ }^{6}$ N. C. Seeman, "DNA in a material world," Nature 421, 427-431 (2003).

${ }^{7}$ Z. Xie, T. Z. Markus, S. R. Cohen, Z. Vager, R. Gutierrez, and R. Naaman, "Spin specific electron conduction through dna oligomers," Nano Lett. 11, 4652-4655 (2011).

${ }^{8}$ R. Naaman and D. H. Waldeck, "Spintronics and chirality: Spin selectivity in electron transport through chiral molecules," Annu. Rev. Phys. Chem. 66, 263-281 (2015).

${ }^{9}$ E. Burzurí, Y. Yamamoto, M. Warnock, X. Zhong, K. Park, A. Cornia, and H. S. J. van der Zant, "Franck-Condon blockade in a single-molecule transistor," Nano Lett. 14, 3191-3196 (2014).

${ }^{10}$ K. J. Franke and J. I. Pascual, "Effects of electron-vibration coupling in transport through single molecules," J. Phys.: Condens. Matter 24, 394002 (2012).

${ }^{11}$ K. J. Franke, G. Schulze, and J. I. Pascual, "Competition of superconducting phenomena and Kondo screening at the nanoscale," Science 332, 940-944 (2011)

${ }^{12}$ J. O. Island, R. Gaudenzi, J. de Bruijckere, E. Burzurí, C. Franco, M. Mas-Torrent, C. Rovira, J. Veciana, T. M. Klapwijk, R. Aguado, and H. S. J. van der Zant, "Proximity-induced Shiba states in a molecular junction," preprint arXiv:1609.08316 (2016).

${ }^{13}$ R. Frisenda, R. Gaudenzi, C. Franco, M. Mas-Torrent, C. Rovira, J. Veciana, I. Alcon, S. T. Bromley, E. Burzurí, and H. S. J. van der Zant, "Kondo effect in a neutral and stable all organic radical single molecule break junction," Nano Lett. 15, 3109-3114 (2015).

${ }^{14}$ M. Koole, J. M. Thijssen, H. Valkenier, J. C. Hummelen, and H. S. van der Zant, "Electric-field control of interfering transport pathways in a single-molecule anthraquinone transistor," Nano Lett. 15, 5569-5573 (2015).

${ }^{15}$ S. Thiele, F. Balestro, R. Ballou, S. Klyatskaya, M. Ruben, and W. Wernsdorfer, "Electrically driven nuclear spin resonance in singlemolecule magnets," Science 344, 1135-1138 (2014).

${ }^{16}$ R. Meservey and P. Tedrow, "Spin-polarized electron tunneling," Phys. Rep. 238, 173-243 (1994).

${ }^{17}$ Z. Nussinov, M. Crommie, and A. Balatsky, "Noise spectroscopy of a single spin with spin-polarized STM,” Phys. Rev. B 68, 085402 (2003).

${ }^{18}$ A. J. Heinrich, J. A. Gupta, C. P. Lutz, and D. M. Eigler, "Single-atom spin-flip spectroscopy," Science 306, 466-469 (2004).

${ }^{19}$ F. Meier, L. Zhou, J. Wiebe, and R. Wiesendanger, "Revealing magnetic interactions from single-atom magnetization curves," Science 320, 82-86 (2008).

${ }^{20}$ X. Chen, Y.-S. Fu, S.-H. Ji, T. Zhang, P. Cheng, X.-C. Ma, X.-L. Zou, W.-H. Duan, J.-F. Jia, and Q.-K. Xue, "Probing superexchange interaction in molecular magnets by spin-flip spectroscopy and microscopy," Phys. Rev. Lett. 101, 197208 (2008).

${ }^{21} \mathrm{R}$. Wiesendanger, "Spin mapping at the nanoscale and atomic scale," Rev. Mod. Phys. 81, 1495 (2009).

${ }^{22}$ K. Petukhov, M. S. Alam, H. Rupp, S. Strömsdörfer, P. Müller, A. Scheurer, R. W. Saalfrank, J. Kortus, A. Postnikov, M. Ruben, L. K. Thompson, and J.-M. Lehn, "STM spectroscopy of magnetic molecules," Coord. Chem. Rev. 253, 2387-2398 (2009).

${ }^{23} \mathrm{~S}$. Kahle et al., "The quantum magnetism of individual manganese-12acetate molecular magnets anchored at surfaces," Nano Lett. 12, 518-521 (2012).

${ }^{24}$ J.-P. Gauyacq, N. Lorente, and F. D. Novaes, "Excitation of local magnetic moments by tunneling electrons," Prog. Surf. Sci. 87, 63-107 (2012).

${ }^{25} \mathrm{M}$. Ternes, "Spin excitations and correlations in scanning tunneling spectroscopy,” New J. Phys. 17, 063016 (2015).

${ }^{26}$ J. A. J. Burgess, L. Malavolti, V. Lanzilotto, M. Mannini, S. Yan, S. Ninova, F. Totti, S. Rolf-Pissarczyk, A. Cornia, R. Sessoli, and S. Loth, "Magnetic fingerprint of individual $\mathrm{Fe}_{4}$ molecular magnets under compression by a scanning tunnelling microscope," Nat. Commun. 6, 8216 (2015).
${ }^{27}$ B. W. Heinrich, L. Braun, J. I. Pascual, and K. J. Franke, "Tuning the magnetic anisotropy of single molecules," Nano Lett. 15, 4024-4028 (2015).

${ }^{28}$ T. Böhler, J. Grebing, A. Mayer-Gindner, H. v Löhneysen, and E. Scheer, "Mechanically controllable break-junctions for use as electrodes for molecular electronics," Nanotechnology 15, S465-S471 (2004).

${ }^{29}$ A. R. Champagne, A. N. Pasupathy, and D. C. Ralph, "Mechanicallyadjustable and electrically-gated single-molecule transistors," Nano Lett. 5, 305 (2005).

${ }^{30}$ D.-H. Chae, J. F. Berry, S. Jung, F. A. Cotton, C. A. Murillo, and Z. Yao, "Vibrational excitations in single trimetal-molecule transistors," Nano Lett. 6, 165-168 (2006).

${ }^{31}$ M. Galperin, M. A. Ratner, and A. Nitzan, "Molecular transport junctions: Vibrational effects," J. Phys.: Condens. Matter 19, 103201 (2007).

${ }^{32}$ J. Hihath, C. R. Arroyo, G. Rubio-Bollinger, N. Tao, and N. Agraït, "Study of electron-phonon interactions in a single molecule covalently connected to two electrodes," Nano Lett. 8, 1673-1678 (2008).

${ }^{33}$ A. K. Hüttel, B. Witkamp, M. Leijnse, M. R. Wegewijs, and H. S. J. van der Zant, "Pumping of vibrational excitations in the Coulomb-blockade regime in a suspended carbon nanotube," Phys. Rev. Lett. 102, 225501 (2009).

${ }^{34}$ J. Parks, A. Champagne, G. Hutchison, S. Flores-Torres, H. Abruna, and D. Ralph, "Tuning the Kondo effect with a mechanically controllable break junction,” Phys. Rev. Lett. 99, 026601 (2007).

${ }^{35}$ J. J. Parks, A. R. Champagne, T. A. Costi, W. W. Shum, A. N. Pasupathy, E. Neuscamman, S. Flores-Torres, P. S. Cornaglia, A. A. Aligia, C. A. Balseiro, G. K.-L. Chan, H. D. Abuña, and D. C. Ralph, "Mechanical control of spin states in spin-1 molecules and the underscreened Kondo effect," Science 328, 1370-1373 (2010).

${ }^{36}$ R. Hanson, L. P. Kouwenhoven, J. R. Petta, S. Tarucha, and L. M. K. Vandersypen, "Spins in few-electron quantum dots," Rev. Mod. Phys. 79, 1217 (2007).

${ }^{37}$ P. Jarillo-Herrero, S. Sapmaz, C. Dekker, L. Kouwenhoven, and H. van der Zant, "Electron-hole symmetry in a semiconducting carbon nanotube quantum dot," Nature 429, 389-392 (2004).

${ }^{38}$ F. H. L. Koppens, J. A. Folk, J. M. Elzerman, R. Hanson, L. H. W. van Beveren, I. T. Vink, H.-P. Tranitz, W. Wegscheider, L. P. Kouwenhoven, and L. M. K. Vandersypen, "Control and detection of singlet-triplet mixing in a random nuclear field," Science 309, 1346-1350 (2005).

${ }^{39}$ P. Jarillo-Herrero, J. Kong, H. S. J. van der Zant, C. Dekker, L. P. Kouwenhoven, and S. De Franceschi, "Electronic transport spectroscopy of carbon nanotubes in a magnetic field," Phys. Rev. Lett. 94, 156802 (2005).

${ }^{40}$ S. Sapmaz, P. Jarillo-Herrero, J. Kong, C. Dekker, L. P. Kouwenhoven, and H. S. J. van der Zant, "Electronic excitation spectrum of metallic carbon nanotubes,” Phys. Rev. B 71, 153402 (2005).

${ }^{41}$ A. K. Hüttel, S. Ludwig, H. Lorenz, K. Eberl, and J. P. Kotthaus, "Direct control of the tunnel splitting in a one-electron double quantum dot," Phys. Rev. B 72, 081310 (2005).

${ }^{42}$ M. R. Gräber, W. A. Coish, C. Hoffmann, M. Weiss, J. Furer, S. Oberholzer, D. Loss, and C. Schönenberger, "Molecular states in carbon nanotube double quantum dots," Phys. Rev. B 74, 075427 (2006).

${ }^{43}$ S. Sapmaz, P. Jarillo-Herrero, L. P. Kouwenhoven, and H. S. J. van der Zant, "Quantum dots in carbon nanotubes," Semicond. Sci. Technol. 21, S52-S63 (2006).

${ }^{44}$ R. Leturcq, C. Stampfer, K. Inderbitzin, L. Durrer, C. Hierold, E. Mariani, M. G. Schultz, F. von Oppen, and K. Ensslin, "Franck-Condon blockade in suspended carbon nanotube quantum dots," Nat. Phys. 5, 327-331 (2009).

${ }^{45}$ S. Andergassen, V. Meden, H. Schoeller, J. Splettstoesser, and M. R. Wegewijs, "Charge transport through single molecules, quantum dots and quantum wires," Nanotechnology 21, 272001 (2010).

${ }^{46}$ F. Haupt, M. Leijnse, H. L. Calvo, L. Classen, J. Splettstoesser, and M. R. Wegewijs, "Heat, molecular vibrations, and adiabatic driving in non-equilibrium transport through interacting quantum dots," Phys. Status Solidi B 250, 2315-2329 (2013).

${ }^{47}$ H. B. Heersche, Z. de Groot, J. A. Folk, H. S. J. van der Zant, C. Romeike, M. R. Wegewijs, L. Zobbi, D. Barreca, E. Tondello, and A. Cornia, "Electron transport through single $\mathrm{Mn}_{12}$ molecular magnets," Phys. Rev. Lett. 96, 206801 (2006).

${ }^{48}$ A. Zyazin, J. van den Berg, E. Osorio, H. van der Zant, N. Konstantinidis, M. Leijnse, M. Wegewijs, F. May, W. Hofstetter, C. Danieli, and A. Cornia, "Electric field controlled magnetic anisotropy in a single molecule," Nano Lett. 10, 3307-3311 (2010). 
${ }^{49}$ E. Burzurí, A. S. Zyazin, A. Cornia, and H. S. J. van der Zant, "Direct observation of magnetic anisotropy in an individual $\mathrm{Fe}_{4}$ single-molecule magnet," Phys. Rev. Lett. 109, 147203 (2012).

${ }^{50}$ M. Misiorny, E. Burzurí, R. Gaudenzi, K. Park, M. Leijnse, M. R. Wegewijs, J. Paaske, A. Cornia, and H. S. J. van der Zant, "Probing transverse magnetic anisotropy by electronic transport through a single-molecule magnet," Phys. Rev. B 91, 035442 (2015).

${ }^{51}$ E. Burzurí, R. Gaudenzi, and H. S. J. van der Zant, "Observing magnetic anisotropy in electronic transport through individual single-molecule magnets," J. Phys.: Condens. Matter 27, 113202 (2015).

${ }^{52}$ J. E. Grose, E. S. Tam, C. Timm, M. Scheloske, B. Ulgut, J. J. Parks, H. D. Abruña, W. Harneit, and D. C. Ralph, "Tunneling spectra of individual magnetic endofullerene molecules," Nat. Mater. 7, 884 (2008).

${ }^{53}$ A. Kogan, G. Granger, M. Kastner, D. Goldhaber-Gordon, and H. Shtrikman, "Singlet-triplet transition in a single-electron transistor at zero magnetic field," Phys. Rev. B 67, 113309 (2003).

${ }^{54}$ N. Craig, J. Taylor, E. Lester, C. Marcus, M. Hanson, and A. Gossard, "Tunable nonlocal spin control in a coupled-quantum dot system," Science 304, 565-567 (2004).

${ }^{55}$ S. Moriyama, T. Fuse, M. Suzuki, Y. Aoyagi, and K. Ishibashi, "Fourelectron shell structures and an interacting two-electron system in carbonnanotube quantum dots," Phys. Rev. Lett. 94, 186806 (2005).

${ }^{56}$ S. Florens, A. Freyn, N. Roch, W. Wernsdorfer, F. Balestro, P. Roura-Bas, and A. Aligia, "Universal transport signatures in two-electron molecular quantum dots: Gate-tunable Hund's rule, underscreened Kondo effect and quantum phase transitions," J. Phys.: Condens. Matter 23, 243202 (2011).

${ }^{57}$ K. Grove-Rasmussen, S. Grap, J. Paaske, K. Flensberg, S. Andergassen, V. Meden, H. I. Jørgensen, K. Muraki, and T. Fujisawa, "Magnetic-field dependence of tunnel couplings in carbon nanotube quantum dots," Phys. Rev. Lett. 108, 176802 (2012).

${ }^{58}$ N. Mason, M. J. Biercuk, and C. M. Marcus, "Local gate control of a carbon nanotube double quantum dot," Science 303, 655-658 (2004).

${ }^{59}$ M. J. Biercuk, S. Garaj, N. Mason, J. M. Chow, and C. M. Marcus, "Gatedefined quantum dots on carbon nanotubes," Nano Lett. 5, 1267-1271 (2005).

${ }^{60}$ J. Hauptmann, J. Paaske, and P. Lindelof, "Electric-field-controlled spin reversal in a quantum dot with ferromagnetic contacts," Nat. Phys. 4, 373-376 (2008).

${ }^{61}$ H. Song, Y. Kim, Y. H. Jang, H. Jeong, M. A. Reed, and T. Lee, "Observation of molecular orbital gating," Nature 462, 1039-1043 (2009).

${ }^{62}$ T. S. Jespersen, K. Grove-Rasmussen, J. Paaske, K. Muraki, T. Fujisawa, J. Nygård, and K. Flensberg, "Gate-dependent spin-orbit coupling in multielectron carbon nanotubes," Nat. Phys. 7, 348-353 (2011).

${ }^{63}$ R. Schleser, T. Ihn, E. Ruh, K. Ensslin, M. Tews, D. Pfannkuche, D. C. Driscoll, and A. C. Gossard, "Cotunneling-mediated transport through excited states in the Coulomb-blockade regime," Phys. Rev. Lett. 94, 206805 (2005).

${ }^{64}$ A. K. Hüttel, M. Poot, B. Witkamp, and H. S. J. van der Zant, "Nanoelectromechanics of suspended carbon nanotubes," New J. Phys. 10, 095003 (2008).

${ }^{65}$ V. N. Golovach and D. Loss, "Transport through a double quantum dot in the sequential tunneling and cotunneling regimes," Phys. Rev. B 69 , 245327 (2004).

${ }^{66}$ J. Aghassi, M. H. Hettler, and G. Schön, "Cotunneling assisted sequential tunneling in multilevel quantum dots," Appl. Phys. Lett. 92, 202101 (2008).

${ }^{67}$ M. C. Lüffe, J. Koch, and F. von Oppen, "Theory of vibrational absorption sidebands in the Coulomb-blockade regime of single-molecule transistors," Phys. Rev. B 77, 125306 (2008).

${ }^{68}$ D. Becker and D. Pfannkuche, "Coulomb-blocked transport through a quantum dot with spin-split level: Increase of differential conductance peaks by spin relaxation," Phys. Rev. B 77, 205307 (2008).

${ }^{69}$ I. Weymann, "Effects of different geometries on the conductance, shot noise, and tunnel magnetoresistance of double quantum dots," Phys. Rev. B 78, 045310 (2008).

${ }^{70}$ M. Leijnse and M. R. Wegewijs, "Kinetic equations for transport through single-molecule transistors," Phys. Rev. B 78, 235424 (2008).

${ }^{71}$ J. M. Thijssen and H. S. J. van der Zant, "Charge transport and single-electron effects in nanoscale systems," Phys. Status Solidi B 245, 1455-1470 (2008).

${ }^{72}$ D. Ferry, S. M. Goodnick, and J. Bird, Transport in Nanostructures, 2nd ed. (Cambridge University Press, Cambridge, 2009).

${ }^{73}$ J. C. Cuevas and E. Scheer, Molecular Electronics: An Introduction to Theory and Experiment, World Scientific Series in Nanoscience and Nanotechnology, Vol. 1 (World Scientific, Singapore, 2010).
${ }^{74}$ S. Tarucha, D. Austing, Y. Tokura, W. van der Wiel, and L. Kouwenhoven, "Direct Coulomb and exchange interaction in artificial atoms," Phys. Rev. Lett. 84, 2485-2488 (2000).

${ }^{75}$ T. H. Oosterkamp, T. Fujisawa, W. G. Van Der Wiel, K. Ishibashi, R. V. Hijman, S. Tarucha, and L. P. Kouwenhoven, "Microwave spectroscopy of a quantum-dot molecule," Nature 395, 873-876 (1998).

${ }^{76}$ P. A. Maksym, H. Imamura, G. P. Mallon, and H. Aoki, "Molecular aspects of electron correlation in quantum dots," J. Phys.: Condens. Matter 12, R299-R334 (2000).

${ }^{77}$ T. Fujisawa, D. G. Austing, Y. Tokura, Y. Hirayama, and S. Tarucha, "Allowed and forbidden transitions in artificial hydrogen and helium atoms," Nature 419, 278-281 (2002).

${ }^{78}$ G. Begemann, D. Darau, A. Donarini, and M. Grifoni, "Symmetry fingerprints of a benzene single-electron transistor: Interplay between Coulomb interaction and orbital symmetry," Phys. Rev. B 77, 201406 (2008).

${ }^{79}$ W. G. van der Wiel, S. De Franceschi, J. M. Elzerman, T. Fujisawa, S. Tarucha, and L. P. Kouwenhoven, "Electron transport through double quantum dots," Rev. Mod. Phys. 75, 1 (2003).

${ }^{80}$ M. Misiorny, I. Weymann, and J. Barnaś, "Spin effects in transport through single-molecule magnets in the sequential and cotunneling regimes," Phys. Rev. B 79, 224420 (2009).

${ }^{81}$ A. McCaskey, Y. Yamamoto, M. Warnock, E. Burzurí, H. S. J. van der Zant, and K. Park, "Electron-vibron coupling effects on electron transport via a single-molecule magnet," Phys. Rev. B 91, 125419 (2015).

${ }^{82}$ R. Vincent, S. Klyatskaya, M. Ruben, W. Wernsdorfer, and F. Balestro, "Electronic read-out of a single nuclear spin using a molecular spin transistor," Nature 488, 357-360 (2012).

${ }^{83}$ M. Ganzhorn, S. Klyatskaya, M. Ruben, and W. Wernsdorfer, "Strong spin-phonon coupling between a single-molecule magnet and a carbon nanotube nanoelectromechanical system," Nat. Nanotechnol. 8, 165-169 (2013).

${ }^{84}$ S. Thiele, R. Vincent, M. Holzmann, S. Klyatskaya, M. Ruben, F. Balestro, and W. Wernsdorfer, "Electrical readout of individual nuclear spin trajectories in a single-molecule magnet spin transistor," Phys. Rev. Lett. 111, 037203 (2013).

${ }^{85}$ D. Cobden, M. Bockrath, P. McEuen, A. Rinzler, and R. Smalley, "Spin splitting and even-odd effects in carbon nanotubes," Phys. Rev. Lett. 81, 681-684 (1998).

${ }^{86}$ T. S. Jespersen, K. Grove-Rasmussen, K. Flensberg, J. Paaske, K. Muraki, T. Fujisawa, and J. Nygård, "Gate-dependent orbital magnetic moments in carbon nanotubes,” Phys. Rev. Lett. 107, 186802 (2011).

${ }^{87}$ P. Weber, H. L. Calvo, J. Bohle, K. Goß, C. Meyer, M. R. Wegewijs, and C. Stampfer, "Switchable coupling of vibrations to two-electron carbonnanotube quantum dot states," Nano Lett. 15, 4417-4422 (2015).

${ }^{88}$ P. G. Piva, G. A. DiLabio, J. L. Pitters, J. Zikovsky, M. Rezeq, S. Dogel, W. A. Hofer, and R. A. Wolkow, "Field regulation of single-molecule conductivity by a charged surface atom," Nature 435, 658-661 (2005).

${ }^{89}$ C. A. Martin, J. M. van Ruitenbeek, and H. S. J. van der Zant, Nanotechnology 21(26), 265201 (2010).

${ }^{90}$ R. Temirov, A. Lassise, F. B. Anders, and F. S. Tautz, "Kondo effect by controlled cleavage of a single-molecule contact," Nanotechnology $\mathbf{1 9}$, 065401 (2008).

${ }^{91}$ C. Toher, R. Temirov, A. Greuling, F. Pump, M. Kaczmarski, G. Cuniberti, M. Rohlfing, and F. S. Tautz, "Electrical transport through a mechanically gated molecular wire," Phys. Rev. B 83, 155402 (2011).

${ }^{92}$ A. Greuling, M. Rohlfing, R. Temirov, F. S. Tautz, and F. B. Anders, "A $b$ initio study of a mechanically gated molecule: From weak to strong correlation," Phys. Rev. B 84, 125413 (2011).

${ }^{93}$ A. Greuling, R. Temirov, B. Lechtenberg, F. B. Anders, M. Rohlfing, and F. S. Tautz, "Spectral properties of a molecular wire in the Kondo regime," Phys. Status Solidi B 250, 2386-2393 (2013).

${ }^{94} \mathrm{C}$. Wagner and R. Temirov, "Tunnelling junctions with additional degrees of freedom: An extended toolbox of scanning probe microscopy," Prog. Surf. Sci. 90, 194-222 (2015).

${ }^{95}$ M. J. Yoo, T. A. Fulton, H. F. Hess, R. L. Willett, L. N. Dunkleberger, R. J. Chichester, L. N. Pfeiffer, and K. W. West, "Scanning single-electron transistor microscopy: Imaging individual charges," Science 276, 579-582 (1997).

${ }^{96}$ C. Wagner, M. F. B. Green, P. Leinen, T. Deilmann, P. Krüger, M. Rohlfing, R. Temirov, and F. S. Tautz, "Scanning quantum dot microscopy," Phys. Rev. Lett. 115, 026101 (2015).

${ }^{97} \mathrm{H}$. Bruus and K. Flensberg, Many-Body Quantum Theory in Condensed Matter Physics (Oxford Graduate Texts, Oxford University Press, Oxford, 2004). 
${ }^{98}$ J. Koch and F. von Oppen, "Franck-Condon blockade and giant Fano factors in transport through single molecules," Phys. Rev. Lett. 94, 206804 (2005).

${ }^{99}$ J. Koch, F. von Oppen, and A. V. Andreev, "Theory of the Franck-Condon blockade regime," Phys. Rev. B 74, 205438 (2006).

${ }^{100}$ F. Reckermann, M. Leijnse, M. R. Wegewijs, and H. Schoeller, "Transport signature of pseudo Jahn-Teller dynamics in a single-molecule transistor," Europhys. Lett. 83, 58001 (2008).

${ }^{101}$ F. Reckermann, M. Leijnse, and M. R. Wegewijs, "Vibrational detection and control of spin in mixed-valence molecular transistors," Phys. Rev. B 79, 075313 (2009).

${ }^{102}$ C. W. J. Beenakker, "Theory of Coulomb-blockade oscillations in the conductance of a quantum dot," Phys. Rev. B 44, 1646 (1991).

${ }^{103}$ K. Kaasbjerg and K. Flensberg, "Image charge effects in single-molecule junctions: Breaking of symmetries and negative-differential resistance in a benzene single-electron transistor," Phys. Rev. B 84, 115457 (2011).

${ }^{104}$ J. Lambe and R. C. Jaklevic, "Molecular vibration spectra by inelastic electron tunneling," Phys. Rev. 165, 821-832 (1968).

${ }^{105}$ A. A. Khajetoorians, J. Wiebe, B. Chilian, S. Lounis, S. Blügel, and R. Wiesendanger, "Atom-by-atom engineering and magnetometry of tailored nanomagnets," Nat. Phys. 8, 497-503 (2012).

${ }^{106}$ J. Brede and R. Wiesendanger, "Spin-resolved characterization of single cobalt phthalocyanine molecules on a ferromagnetic support," Phys. Rev. B 86, 184423 (2012).

${ }^{107}$ A. A. Khajetoorians, B. Baxevanis, C. Hübner, T. Schlenk, S. Krause, T. O. Wehling, S. Lounis, A. Lichtenstein, D. Pfannkuche, J. Wiebe, and R. Wiesendanger, "Current-driven spin dynamics of artificially constructed quantum magnets," Science 339, 55-59 (2013).

${ }^{108}$ B. Bryant, A. Spinelli, J. J. T. Wagenaar, M. Gerrits, and A. F. Otte, "Local control of single atom magnetocrystalline anisotropy," Phys. Rev. Lett. 111, 127203 (2013)

${ }^{109}$ I. G. Rau, S. Baumann, S. Rusponi, F. Donati, S. Stepanow, L. Gragnaniello, J. Dreiser, C. Piamonteze, F. Nolting, S. Gangopadhyay, O. R. Albertini, R. M. Macfarlane, C. P. Lutz, B. A. Jones, P. Gambardella, A. J. Heinrich, and H. Brune, "Reaching the magnetic anisotropy limit of a 3D metal atom," Science 344, 988-992 (2014).

${ }^{110}$ P. Hapala, G. Kichin, C. Wagner, F. S. Tautz, R. Temirov, and P. Jelínek, "Mechanism of high-resolution STM/AFM imaging with functionalized tips," Phys. Rev. B 90, 085421 (2014).

${ }^{111}$ C. Wagner, N. Fournier, V. G. Ruiz, C. Li, K. Müllen, M. Rohlfing, A. Tkatchenko, R. Temirov, and F. S. Tautz, "Non-additivity of moleculesurface van der Waals potentials from force measurements," Nat. Commun. 5, 5568 (2014).

${ }^{112}$ M. Bazarnik, B. Bugenhagen, M. Elsebach, E. Sierda, A. Frank, M. H. Prosenc, and R. Wiesendanger, "Toward tailored all-spin molecular devices," Nano Lett. 16, 577-582 (2016).

${ }^{113}$ C. Hirjibehedin, C. Lutz, and A. Heinrich, "Spin coupling in engineered atomic structures," Science 312, 1021-1024 (2006).

${ }^{114}$ C. Hirjibehedin, C. Lin, A. Otte, M. Ternes, C. Lutz, B. Jones, and A. Heinrich, "Large magnetic anisotropy of a single atomic spin embedded in a surface molecular network," Science 317, 1199-1203 (2007).

${ }^{115}$ D. Serrate, P. Ferriani, Y. Yoshida, S.-W. Hla, M. Menzel, K. von Bergmann, S. Heinze, A. Kubetzka, and R. Wiesendanger, "Imaging and manipulating the spin direction of individual atoms," Nat. Nanotechnol. 5, 350 (2010).

${ }^{116}$ S. Baumann, F. Donati, S. Stepanow, S. Rusponi, W. Paul, S. Gangopadhyay, I. G. Rau, G. E. Pacchioni, L. Gragnaniello, M. Pivetta, J. Dreiser, C. Piamonteze, C. P. Lutz, R. M. Macfarlane, B. A. Jones, P. Gambardella, A. J. Heinrich, and H. Brune, "Origin of perpendicular magnetic anisotropy and large orbital moment in Fe atoms on MgO," Phys. Rev. Lett. 115, 237202 (2015).

${ }^{117}$ C. Weiss, C. Wagner, C. Kleimann, M. Rohlfing, F. S. Tautz, and R. Temirov, "Imaging Pauli repulsion in scanning tunneling microscopy," Phys. Rev. Lett. 105, 086103 (2010).

${ }^{118}$ T. Esat, T. Deilmann, B. Lechtenberg, C. Wagner, P. Krüger, R. Temirov, F. B. Anders, M. Rohlfing, and F. S. Tautz, "Transfering spin into an extended $\pi$ orbital of a large molecule," Phys. Rev. B 91, 144415 (2015).

${ }^{119}$ N. Atodiresei, V. Caciuc, P. Lazić, and S. Blügel, "Chemical versus van der waals interaction: The role of the heteroatom in the flat absorption of aromatic molecules $\mathrm{C}_{6} \mathrm{H}_{6}, \mathrm{C}_{5} \mathrm{NH}_{5}$, and $\mathrm{C}_{4} \mathrm{~N}_{2} \mathrm{H}_{4}$ on the $\mathrm{Cu}(110)$ surface," Phys. Rev. Lett. 102, 136809 (2009).

${ }^{120}$ S. Loth, K. von Bergmann, M. Ternes, A. Otte, C. Lutz, and A. Heinrich, "Controlling the state of quantum spins with electric currents," Nat. Phys. 6, 340-344 (2010).
${ }^{121}$ A. A. Khajetoorians, S. Lounis, B. Chilian, A. T. Costa, L. Zhou, D. L. Mills, J. Wiebe, and R. Wiesendanger, "Itinerant nature of atommagnetization excitation by tunneling electrons," Phys. Rev. Lett. 106, 037205 (2011).

${ }^{122}$ S. Yan, D.-J. Choi, J. A. J. Burgess, S. Rolf-Pissarczyk, and S. Loth, "Threedimensional mapping of single-atom magnetic anisotropy," Nano Lett. 15, 1938-1942 (2015).

${ }^{123}$ S. Yan, D.-J. Choi, J. A. J. Burgess, S. Rolf-Pissarczyk, and S. Loth, “Control of quantum magnets by atomic exchange bias," Nat. Nanotechnol. 10, 40-45 (2015).

${ }^{124}$ A. A. Khajetoorians, M. Steinbrecher, M. Ternes, M. Bouhassoune, M. dos Santos Dias, S. Lounis, J. Wiebe, and R. Wiesendanger, "Tailoring the chiral magnetic interaction between two individual atoms," Nat. Commun. 7, 10620 (2016).

${ }^{125}$ M. Steinbrecher, A. Sonntag, M. dos Santos Dias, M. Bouhassoune, S. Lounis, J. Wiebe, R. Wiesendanger, and A. A. Khajetoorians, "Absence of a spin-signature from a single Ho adatom as probed by spin-sensitive tunneling," Nat. Commun. 7, 10454 (2016).

${ }^{126}$ A. Otte, M. Ternes, K. von Bergmann, S. Loth, H. Brune, C. Lutz, C. Hirjibehedin, and A. Heinrich, "The role of magnetic anisotropy in the Kondo effect," Nat. Phys. 4, 847-850 (2008).

${ }^{127}$ S. Loth, C. Lutz, and A. Heinrich, "Spin-polarized spin excitation spectroscopy," New J. Phys. 12, 125021 (2010).

${ }^{128}$ C. F. Hirjibehedin and Y. Wang, "Recent advances in scanning tunneling microscopy and spectroscopy," J. Phys.: Condens. Matter 26, 394001-394010 (2014).

${ }^{129}$ J. Repp, G. Meyer, S. M. Stojković, A. Gourdon, and C. Joachim, "Molecules on insulating films: Scanning-tunneling microscopy imaging of individual molecular orbitals," Phys. Rev. Lett. 94, 026803 (2005).

${ }^{130}$ S. De Franceschi, S. Sasaki, J. Elzerman, W. van der Wiel, S. Tarucha, and L. Kouwenhoven, "Electron cotunneling in a semiconductor quantum dot," Phys. Rev. Lett. 86, 878-881 (2001).

${ }^{131}$ D. M. Zumbühl, C. M. Marcus, M. P. Hanson, and A. C. Gossard, "Cotunneling spectroscopy in few-electron quantum dots," Phys. Rev. Lett. 93, 256801 (2004)

${ }^{132}$ G. Katsaros, P. Spathis, M. Stoffel, F. Fournel, M. Mongillo, V. Bouchiat, F. Lefloch, A. Rastelli, O. G. Schmidt, and S. De Franceschi, Nat. Nanotechnol. 5, 458 (2010).

${ }^{133}$ M. R. Wegewijs and Y. V. Nazarov, "Inelastic co-tunneling through an excited state of a quantum dot," e-print arXiv:cond-mat/0103579.

${ }^{134}$ U. Hartmann and F. K. Wilhelm, "Nonlinear cotunneling through an artificial molecule,” Phys. Rev. B 67, 161307 (2003).

${ }^{135}$ D. V. Averin and Y. V. Nazarov, "Virtual electron diffusion during quantum tunneling of the electric charge," Phys. Rev. Lett. 65, 2446 (1990).

${ }^{136}$ Single Charge Tunneling: Coulomb Blockade Phenomena in Nanostructures, NATO Advanced Science Institutes Series Vol. 294, edited by H. Grabert and M. H. Devoret (Springer Science \& Business Media, New York, 1992).

${ }^{137}$ B. C. Stipe, M. A. Rezaei, and W. Ho, "Single-molecule vibrational spectroscopy and microscopy," Science 280, 1732-1735 (1998).

${ }^{138}$ L. H. Yu, Z. K. Keane, J. W. Ciszek, L. Cheng, M. P. Stewart, J. M. Tour, and D. Natelson, "Inelastic electron tunneling via molecular vibrations in single-molecule transistors," Phys. Rev. Lett. 93, 266802 (2004).

${ }^{139}$ Y. Kim, H. Song, F. Strigl, H.-F. Pernau, T. Lee, and E. Scheer, "Conductance and vibrational states of single-molecule junctions controlled by mechanical stretching and material variation," Phys. Rev. Lett. 106, 196804 (2011).

${ }^{140}$ M. Bürkle, J. K. Viljas, T. J. Hellmuth, E. Scheer, F. Weigend, G. Schön, and F. Pauly, "Influence of vibrations on electron transport through nanoscale contacts," Phys. Status Solidi B 250, 2468-2480 (2013).

${ }^{141}$ T. Böhler, A. Edtbauer, and E. Scheer, "Conductance of individual $\mathrm{C}_{60}$ molecules measured with controllable gold electrodes," Phys. Rev. B 76, 125432 (2007).

${ }^{142}$ M. Herz and E. Scheer, "Force-noise spectroscopy by tunneling current deflection sensing," Appl. Phys. Lett. 108, 023103 (2016).

${ }^{143}$ M. L. Perrin, C. J. O. Verzijl, C. A. Martin, A. J. Shaikh, R. Eelkema, J. H. van Esch, J. M. van Ruitenbeek, J. M. Thijssen, H. S. J. van der Zant, and D. Dulić, "Large tunable image-charge effects in single-molecule junctions," Nat. Nanotechnol. 8, 282-287 (2013).

${ }^{144}$ J. König, H. Schoeller, and G. Schön, "Cotunneling at resonance for the single-electron transistor,” Phys. Rev. Lett. 78, 4482-4485 (1997).

${ }^{145}$ S. Schmaus, V. Koerting, J. Paaske, T. S. Jespersen, J. Nygård, and P. Wölfle, "Nonequilibrium cotunneling through a three-level quantum dot,” Phys. Rev. B 79, 045105 (2009). 
${ }^{146}$ J. Paaske, A. Rosch, P. Wolfle, N. Mason, C. M. Marcus, and J. Nygard, "Non-equilibrium singlet-triplet Kondo effect in carbon nanotubes," Nat. Phys. 2, 460-464 (2006).

${ }^{147}$ M. Wagner, Unitary Transformations in Solid State Physics, Modern Problems in Condensed Matter Sciences Vol. 15 (North-Holland Physics Publishing, Amsterdam, 1986).

${ }^{148}$ J. Appelbaum, “"s-d' exchange model of zero-bias tunneling anomalies," Phys. Rev. Lett. 17, 91-95 (1966).

${ }^{149}$ J. Appelbaum, "Exchange model of zero-bias tunneling anomalies," Phys. Rev. 154, 633 (1967).

${ }^{150}$ J. R. Schrieffer and P. A. Wolff, "Relation between the Anderson and Kondo Hamiltonians," Phys. Rev. 149, 491 (1966).

${ }^{151}$ J. Schrieffer, "The Kondo effect-The link between magnetic and nonmagnetic impurities in metals?,” J. Appl. Phys. 38, 1143-1150 (1967).

${ }^{152}$ S. Bravyi, D. P. DiVincenzo, and D. Loss, "Schrieffer-Wolff transformation for quantum many-body systems," Ann. Phys. 326, 2793-2826 (2011).

${ }^{153}$ We note that to transfer a single electron through the molecule, two electrons are needed: one enters the molecule, creating a virtual intermediate state, and one exits the molecule, producing the final state. In general, these electrons not necessarily have to be added to the same spin and/or orbital state on the molecule.

${ }^{154}$ This result can be understood in more detail from Eq. (16) as explained in Refs. 25 and 120: The Golden Rule applied to the new effective coupling $\mathcal{H}^{\mathrm{A}}$ gives transition rates which involve matrix elements, $\left\langle S^{\prime} M^{\prime}, s^{\prime} m\left|\mathcal{H}^{\mathrm{A}}\right| S M, s m\right\rangle$ where we omit further quantum numbers unrelated to spin. Due to the factorization of the states $|S M, s m\rangle=|S M\rangle \otimes|s m\rangle$ into the molecule $(|S M\rangle)$ and electrode part $(|s m\rangle)$ in this approximation, the matrix element have factors $\left\langle S^{\prime} M^{\prime}|\mathbf{S}| S M\right\rangle$, which is zero unless $\Delta S=0$ and $|\Delta M|=0,1$, and $\left\langle S^{\prime} M^{\prime}|N| S M\right\rangle$, which is zero unless $\Delta S=\Delta M=0$.

${ }^{155}$ K. Kikoin, M. Kiselev, and Y. Avishai, Dynamical Symmetries in Nanophysics, Nanophysics, Nanoclusters and Nanodevices (NOVA Science Publisher, New York, USA, 2006).

${ }^{156}$ L. Hirst, "Theory of the coupling between conduction electrons and moments of 3d and 4f ions in metals," Adv. Phys. 27, 231 (1978).

${ }^{157} \mathrm{M}$. Eto and Y. V. Nazarov, "Enhancement of Kondo effect in quantum dots with an even number of electrons," Phys. Rev. Lett. 85, 1306 (2000).

${ }^{158}$ B. Sothmann and J. König, "Nonequilibrium current and noise in inelastic tunneling through a magnetic atom," New J. Phys. 12, 083028 (2010).

${ }^{159}$ A. Hurley, N. Baadji, and S. Sanvito, "Perturbative approach to the Kondo effect in magnetic atoms on nonmagnetic substrates," Phys. Rev. B 84, 115435 (2011).

${ }^{160}$ A. Hurley, N. Baadji, and S. Sanvito, "Bias asymmetry in the conductance profile of magnetic ions on surfaces probed by scanning tunneling microscopy," Phys. Rev. B 86, 125411 (2012).

${ }^{161}$ B. Schweflinghaus, M. dos Santos Dias, A. T. Costa, and S. Lounis, "Renormalization of electron self-energies via their interaction with spin excitations: A first principles investigation,” Phys. Rev. B 89, 235439 (2014).

162 A. Rosch, J. Paaske, J. Kroha, and P. Wölfle, "Nonequilibrium transport through a kondo dot in a magnetic field: Perturbation theory and poor man's scaling," Phys. Rev. Lett. 90, 076804 (2003).

${ }^{163}$ J. Paaske, A. Rosch, and P. Wölfle, "Nonequilibrium transport through a kondo dot in a magnetic field: Perturbation theory," Phys. Rev. B 69, 155330 (2004).

${ }^{164}$ H. Schoeller and F. Reininghaus, "Real-time renormalization group in frequency space: A 2-loop analysis of the nonequilibrium anisotropic Kondo model at finite magnetic field," Phys. Rev. B 80, 045117 (2009).

${ }^{165}$ R. Saptsov and M. Wegewijs, "Fermionic superoperators for zerotemperature nonlinear transport: Real-time perturbation theory and renormalization group for Anderson quantum dots," Phys. Rev. B 86, 235432 (2012).

${ }^{166}$ I. L. Aleiner, P. W. Brouwer, and L. I. Glazman, "Quantum effects in Coulomb blockade," Phys. Rep. 358, 309-440 (2002).

${ }^{167}$ M. Pustilnik and L. I. Glazman, "Kondo effect in quantum dots," J. Phys.: Condens. Matter 16, R513-R537 (2004).

${ }^{168}$ L. I. Glazman and M. Pustilnik, "Low temperature transport through a quantum dot," in Nanophysics: Coherence and Transport, Vol. 81, École de Physique des Les Houches, edited by H. Bouchiat, Y. Gefen, S. Guéron, G. Montambaux, and J. Dalibard (Elsevier, Amsterdam, 2005), pp. 427-478.

${ }^{169} \mathrm{H}$. Schoeller, "A perturbative nonequilibrium renormalization group method for dissipative quantum mechanics,” Eur. Phys. J.: Spec. Top. 168, 179-266 (2009).
${ }^{170} \mathrm{P}$. Fritsch and S. Kehrein, "Non-equilibrium scaling analysis of the Kondo model with voltage bias," Ann. Phys. 324, 1105-1135 (2009).

${ }^{171}$ J. Eckel, F. Heidrich-Meisner, S. Jakobs, M. Thorwart, M. Pletyukhov, and R. Egger, "Comparative study of theoretical methods for non-equilibrium quantum transport," New J. Phys. 12, 043042 (2010).

${ }^{172}$ J. Paaske, A. Rosch, J. Kroha, and P. Wölfle, "Nonequilibrium transport through a Kondo dot: Decoherence effects," Phys. Rev. B 70, 155301 (2004).

${ }^{173}$ H. B. Heersche, Z. de Groot, J. A. Folk, L. P. Kouwenhoven, H. S. J. van der Zant, A. A. Houck, J. Labaziewicz, and I. L. Chuang, "Kondo effect in the presence of magnetic impurities," Phys. Rev. Lett. 96, 017205 (2006).

${ }^{174}$ G. D. Scott and D. Natelson, "Kondo resonances in molecular devices," ACS Nano 4, 3560-3579 (2010).

${ }^{175}$ T. Komeda, H. Isshiki, J. Liu, Y.-F. Zhang, N. Lorente, K. Katoh, B. K. Breedlove, and M. Yamashita, "Observation and electric current control of a local spin in a single-molecule magnet," Nat. Commun. 2, 217 (2011).

${ }^{176}$ C. Romeike, M. R. Wegewijs, W. Hofstetter, and H. Schoeller, "Quantumtunneling-induced Kondo effect in single molecular magnets," Phys. Rev. Lett. 96, 196601 (2006).

${ }^{177}$ M. N. Leuenberger and E. R. Mucciolo, "Berry-phase oscillations of the Kondo effect in single-molecule magnets," Phys. Rev. Lett. 97, 126601 (2006).

${ }^{178}$ C. Romeike, M. R. Wegewijs, W. Hofstetter, and H. Schoeller, "Erratum: Kondo-transport spectroscopy of single molecule magnets [Phys. Rev. Lett. 97, 206601 (2006)]," Phys. Rev. Lett. 106, 019902 (2011).

${ }^{179}$ M. R. Wegewijs, C. Romeike, H. Schoeller, and W. Hofstetter, "Corrigendum: Magneto-transport through single-molecule magnets: Kondo-peaks, zero-bias dips, molecular symmetry and Berry's phase [New. J. Phys. 9, 344 (2007)]," New. J. Phys. 13, 079501 (2011).

${ }^{180}$ R. Žitko, R. Peters, and T. Pruschke, "Properties of anisotropic magnetic impurities on surfaces,” Phys. Rev. B 78, 224404 (2008).

${ }^{181}$ R. Žitko, R. Peters, and T. Pruschke, "Splitting of the Kondo resonance in anisotropic magnetic impurities on surfaces," New J. Phys. 11, 053003 (2009).

${ }^{182}$ J. Fernández-Rossier, "Theory of single-spin inelastic tunneling spectroscopy,” Phys. Rev. Lett. 102, 256802 (2009).

${ }^{183}$ R. Žitko and T. Pruschke, "Many-particle effects in adsorbed magnetic atoms with easy-axis anisotropy: The case of $\mathrm{Fe}$ on the $\mathrm{CuN} / \mathrm{Cu}(100)$ surface," New J. Phys. 12, 063040 (2010).

${ }^{184} \mathrm{~F}$. Elste and C. Timm, "Resonant and Kondo tunneling through molecular magnets," Phys. Rev. B 81, 24421 (2010).

${ }^{185}$ M. Misiorny, I. Weymann, and J. Barnaś, "Interplay of the Kondo effect and spin-polarized transport in magnetic molecules, adatoms, and quantum dots," Phys. Rev. Lett. 106, 126602 (2011).

${ }^{186} \mathrm{M}$. Misiorny, I. Weymann, and J. Barnaś, "Influence of magnetic anisotropy on the Kondo effect and spin-polarized transport through magnetic molecules, adatoms, and quantum dots," Phys. Rev. B 84, 035445 (2011).

${ }^{187}$ F. Delgado and J. Fernández-Rossier, "Cotunneling theory of atomic spin inelastic electron tunneling spectropy," Phys. Rev. B 84, 045439 (2011).

${ }^{188} \mathrm{M}$. Misiorny and I. Weymann, "Transverse anisotropy effects on spinresolved transport through large-spin molecules,” Phys. Rev. B 90, 235409 (2014).

${ }^{189}$ M. Ternes, A. Heinrich, and W.-D. Schneider, "Spectroscopic manifestations of the Kondo effect on single adatoms," J. Phys.: Condens. Matter 21, 053001 (2009).

${ }^{190}$ H. Brune and P. Gambardella, "Magnetism of individual atoms adsorbed on surfaces," Surf. Sci. 603, 1812-1830 (2009).

${ }^{191}$ S. Lounis, B. Schweflinghaus, M. dos Santos Dias, M. Bouhassoune, R. B. Muniz, and A. T. Costa, "Theoretical probing of inelastic spinexcitations in adatoms on surfaces," Surf. Sci. 630, 317 (2014).

${ }^{192}$ S. Koller, M. Grifoni, M. Leijnse, and M. R. Wegewijs, "Density-operator approaches to transport through interacting quantum dots: Simplifications in fourth-order perturbation theory," Phys. Rev. B 82, 235307 (2010).

${ }^{193}$ These rates can be brought into a form similar to those obtained in the ASW and $\mathcal{T}$-matrix approach, allowing some of advantages of the latter to be exploited.

${ }^{194}$ M. Turek and K. A. Matveev, Phys. Rev. B 65, 115332 (2002).

${ }^{195}$ G. Begemann, S. Koller, M. Grifoni, and J. Paaske, "Inelastic cotunneling in quantum dots and molecules with weakly broken degeneracies," Phys. Rev. B 82, 045316 (2010).

${ }^{196} \mathrm{C}$. Timm, "Tunneling through molecules and quantum dots: Masterequation approaches,” Phys. Rev. B 77, 195416 (2008).

${ }^{197}$ C. Timm, Phys. Rev. B 83, 115416 (2011). 
${ }^{198}$ J. König, J. Schmid, H. Schoeller, and G. Schön, "Resonant tunneling through ultrasmall quantum dots: Zero-bias anomalies, magnetic-field dependence, and boson-assisted transport," Phys. Rev. B 54, 16820 (1996).

${ }^{199}$ J. König, H. Schoeller, and G. Schön, "Cotunneling and renormalization effects for the single-electron transistor," Phys. Rev. B 58, 7882 (1998).

${ }^{200}$ N. Gergs, R. Saptsov, M. R. Wegewijs, and D. Schuricht, "Heat-currents through interacting nanostructures beyond weak tunnelling: Nonconservation of local energy, multi-orbital effects and attractive interaction" (unpublished).

${ }^{201}$ E. A. Osorio, K. Moth-Poulsen, H. van der Zant, J. Paaske, P. Hedegård, K. Flensberg, J. Bendix, and T. Bjørnholm, "Electrical manipulation of spin states in a single electrostatically gated transition-metal complex," Nano Lett. 10, 105 (2010).

${ }^{202}$ A. Eliasen, J. Paaske, K. Flensberg, S. Smerat, M. Leijnse, M. R. Wegewijs, H. I. Jørgensen, M. Monthioux, and J. Nygård, “Transport via coupled states in a c 60 peapod quantum dot," Phys. Rev. B 81, 155431 (2010).

${ }^{203}$ C. Stevanato, M. Leijnse, K. Flensberg, and J. Paaske, "Finite-bias conductance anomalies at a singlet-triplet crossing," Phys. Rev. B 86, 165427 (2012).

${ }^{204}$ M. Leijnse, M. R. Wegewijs, and M. H. Hettler, "Pair tunneling resonance in the single-electron transport regime," Phys. Rev. Lett. 103, 156803 (2009).

${ }^{205}$ F. D. M. Haldane, "Hartree-Fock study of the Anderson model coupled to a boson field; mixed valence states," Phys. Rev. B 15, 281-289 (1977).

${ }^{206}$ J. Koch, M. E. Raikh, and F. von Oppen, "Pair tunneling through single molecules," Phys. Rev. Lett. 96, 056803 (2006).

${ }^{207}$ M.-J. Hwang, M.-S. Choi, and R. López, "Pair tunneling and shot noise through a single molecule in a strong electron-phonon coupling regime," Phys. Rev. B 76, 165312 (2007).

${ }^{208}$ J. Koch, E. Sela, Y. Oreg, and F. von Oppen, "Nonequilibrium chargeKondo transport through negative-u molecules," Phys. Rev. B 75, 195402 (2007).

${ }^{209}$ E. Sela, H.-S. Sim, Y. Oreg, M. E. Raikh, and F. von Oppen, "Electronpair resonance in the Coulomb blockade," Phys. Rev. Lett. 100, 056809 (2008).

${ }^{210}$ D. H. Evans and M. W. Lehmann, "Two-electron reactions in organic and organometallic electrochemistry," Acta Chem. Scand. 53, 765 (1999).

${ }^{211}$ G. Cheng, M. Tomczyk, S. Lu, J. P. Veazey, M. Huang, P. Irvin, S. Ryu, H. Lee, C.-B. Eom, C. S. Hellberg, and J. Levy, "Electron pairing without superconductivity," Nature 528, 196-199 (2015).

${ }^{212}$ A. Hamo, A. Benyamini, I. Shapir, I. Khivrich, J. Waissman, K. Kaasbjerg, Y. Oreg, F. von Oppen, and S. Ilani, "Electron attraction mediated by Coulomb repulsion," Nature 535, 395 (2016).

${ }^{213}$ M. Nilsson, L. Namazi, S. Lehmann, M. Leijnse, K. A. Dick, and C. Thelander, "Electron-hole interactions in coupled InAs-GaSb quantum dots based on nanowire crystal phase templates," Phys. Rev. B 94, 115313 (2016).

${ }^{214} \mathrm{~B}$. Sothmann and J. König, "Transport through quantum-dot spin valves containing magnetic impurities," Phys. Rev. B 82, 245319 (2010).

${ }^{215}$ M. Misiorny, M. Hell, and M. R. Wegewijs, "Spintronic magnetic anisotropy," Nat. Phys. 9, 801-805 (2013).

${ }^{216} \mathrm{M}$. Braun, J. König, and J. Martinek, "Theory of transport through quantum-dot spin valves in the weak-coupling regime," Phys. Rev. B 70, 195345 (2004).

${ }^{217}$ J. König and Y. Gefen, "Coherence and partial coherence in interacting electron systems,” Phys. Rev. Lett. 86, 3855-3858 (2001).

${ }^{218}$ B. Wunsch, M. Braun, J. König, and D. Pfannkuche, "Probing level renormalization by sequential transport through double quantum dots," Phys. Rev. B 72, 205319 (2005).

${ }^{219}$ A. Donarini, M. Grifoni, and K. Richter, "Dynamical symmetry breaking in transport through molecules," Phys. Rev. Lett. 97, 166801 (2006).

${ }^{220}$ R. Härtle and A. J. Millis, "Formation of nonequilibrium steady states in interacting double quantum dots: When coherences dominate the charge distribution," Phys. Rev. B 90, 245426 (2014).

${ }^{221} \mathrm{~S}$. Wenderoth, J. Bätge, and R. Härtle, "Sharp peaks in the conductance of a double quantum dot and a quantum-dot spin valve at high temperatures: A hierarchical quantum master equation approach,” Phys. Rev. B 94, 121303 (2016).

${ }^{222}$ A. Donarini, B. Siegert, S. Sobczyk, and M. Grifoni, "Topographical fingerprints of many-body interference in STM junctions on thin insulating films," Phys. Rev. B 86, 155451 (2012).

${ }^{223}$ A. Donarini, G. Begemann, and M. Grifoni, "All-electric spin control in interference single electron transistors,” Nano Lett. 9, 2897 (2009).
${ }^{224}$ A. Donarini, G. Begemann, and M. Grifoni, "Interference effects in the Coulomb blockade regime: Current blocking and spin preparation in symmetric nanojunctions," Phys. Rev. B 82, 125451 (2010).

${ }^{225}$ M. M. E. Baumgärtel, M. Hell, S. Das, and M. R. Wegewijs, "Transport and accumulation of spin anisotropy,” Phys. Rev. Lett. 107, 087202 (2011).

${ }^{226} \mathrm{M}$. Misiorny, I. Weymann, and J. Barnaś, "Underscreened Kondo effect in $S=1$ magnetic quantum dots: Exchange, anisotropy and temperature effects," Phys. Rev. B 86, 245415 (2012).

${ }^{227}$ J. C. Oberg, M. R. Calvo, F. Delgado, M. Moro-Lagares, D. Serrate, D. Jacob, J. Fernández-Rossier, and C. F. Hirjibehedin, "Control of singlespin magnetic anisotropy by exchange coupling," Nat. Nanotechnol. 9, 64-68 (2014).

${ }^{228}$ F. Delgado, C. F. Hirjibehedin, and J. Fernández-Rossier, "Consequences of Kondo exchange on quantum spins," Surf. Sci. 630, 337-342 (2014).

${ }^{229}$ P. Jacobson, T. Herden, M. Muenks, G. Laskin, O. Brovko, V. Stepanyuk, M. Ternes, and K. Kern, "Quantum engineering of spin and anisotropy in magnetic molecular junctions," Nat. Commun. 6, 8536 (2015).

${ }^{230}$ M. Hell, B. Sothmann, M. Leijnse, M. Wegewijs, and J. König, "Spin resonance without spin splitting," Phys. Rev. B 91, 195404 (2015).

${ }^{231}$ S. Accorsi, A. L. Barra, A. Caneschi, G. Chastanet, A. Cornia, A. C. Fabretti, D. Gatteschi, C. Mortalo, E. Olivieri, F. Parenti, P. Rosa, R. Sessoli, L. Sorace, W. Wernsdorfer, and L. Zobbi, "Tuning anisotropy barriers in a family of tetrairon(III) single-molecule magnets with an $s=5$ ground state," J. Am. Chem. Soc. 128, 4742-4755 (2006).

${ }^{232}$ R. Gaudenzi, E. Burzurí, D. Reta, I. d. P. R. Moreira, S. T. Bromley, C. Rovira, J. Veciana, and H. S. J. van der Zant, "Exchange coupling inversion in a high-spin organic triradical molecule," Nano Lett. 16, 2066-2071 (2016).

${ }^{233}$ E. A. Osorio, K. O’Neill, M. R. Wegewijs, N. Stuhr-Hansen, J. Paaske, T. Bjørnholm, and H. S. van der Zant, "Electronic excitations of a single molecule contacted in a three-terminal configuration," Nano Lett. 7, 3336 (2007).

${ }^{234}$ A. K. Hüttel, H. Qin, A. W. Holleitner, R. H. Blick, K. Neumaier, D. Weinmann, K. Eberl, and J. P. Kotthaus, "Spin blockade in ground-state resonance of a quantum dot," Europhys. Lett. 62, 712 (2003).

${ }^{235}$ M. Ciorga, A. S. Sachrajda, P. Hawrylak, C. Gould, P. Zawadzki, S. Jullian, Y. Feng, and Z. Wasilewski, "Addition spectrum of a lateral dot from Coulomb and spin-blockade spectroscopy," Phys. Rev. B 61, R16315 (2000).

${ }^{236}$ A. C. Johnson, J. R. Petta, C. M. Marcus, M. P. Hanson, and A. C. Gossard, "Singlet-triplet spin blockade and charge sensing in a few-electron double quantum dot," Phys. Rev. B 72, 165308 (2005).

${ }^{237}$ D. Weinmann, W. Häusler, W. Pfaff, B. Kramer, and U. Weiss, "Spin blockade in non-linear transport through quantum dots," Europhys. Lett. 26, 467 (1994).

${ }^{238}$ D. Weinmann, W. Häusler, and B. Kramer, "Spin blockades in linear and nonlinear transport through quantum dots," Phys. Rev. Lett. 74, 984-987 (1995).

${ }^{239}$ D. Weinmann, "Spin blockades in the transport through quantum dots," Lect. Notes Phys. 630, 289-301 (2003).

${ }^{240}$ C. Romeike, M. R. Wegewijs, and H. Schoeller, "Spin quantum tunneling in single molecular magnets: Fingerprints in transport spectroscopy of current and noise," Phys. Rev. Lett. 96, 196805 (2006).

${ }^{241}$ C. Romeike, M. R. Wegewijs, M. Ruben, W. Wenzel, and H. Schoeller, "Charge-switchable molecular magnet and spin blockade of tunneling," Phys. Rev. B 75, 064404 (2007).

${ }^{242}$ We note that if spin multiplets are internally split, for example, due to magnetic anisotropy, then the spectroscopy gives more information about the absolute value of $S_{g}^{N+1}$. This is exploited, e.g., in the gate-voltage spectroscopy of single-molecule magnets. ${ }^{49,50}$

${ }^{243}$ K. O'Neill, E. A. Osorio, and H. S. J. van der Zant, "Self-breaking in planar few-atom Au constrictions for nanometer-spaced electrodes," Appl. Phys. Lett. 90, 133109 (2007).

${ }^{244}$ R. Gaudenzi, J. O. Island, J. de Bruijckere, E. Burzurí, T. M. Klapwijk, and H. S. J. van der Zant, "Superconducting molybdenum-rhenium electrodes for single-molecule transport studies," Appl. Phys. Lett. 106, 222602 (2015).

${ }^{245}$ Physisorption is the dominant binding mechanism for the molecular species used here, given no chemically active linking groups are present. Chemisorption is the other common alternative used in molecular electronics where a chemical bond forms between the molecule and the electrode.

${ }^{246}$ R. Frisenda and H. S. J. van der Zant, Phys. Rev. Lett. 117, 126809 (2016). 
${ }^{247}$ F. Haque, M. Langhirt, E. Del Barco, T. Taguchi, and G. Christou, "Magnetic field dependent transport through a $\mathrm{Mn}_{4}$ single-molecule magnet," J. Appl. Phys. 109, 07B112 (2011).

${ }^{248}$ J. J. Henderson, C. M. Ramsey, E. del Barco, A. Mishra, and G. Christou, "Fabrication of nanogapped single-electron transistors for transport studies of individual single-molecule magnets," J. Appl. Phys. 101, 09E102 (2007).

${ }^{249}$ M.-H. Jo, J. Grose, K. Baheti, M. Deshmukh, J. Sokol, E. Rumberger, D. Hendrickson, R. Jeffrey, H. Park, and D. Ralph, "Signatures of molecular magnetism in single-molecule transport spectroscopy," Nano Lett. 6, 2014-2020 (2006).

${ }^{250}$ A. Candini, S. Klyatskaya, M. Ruben, W. Wernsdorfer, and M. Affronte, "Graphene spintronic devices with molecular nanomagnets," Nano Lett. 11, 2634-2639 (2011).

${ }^{251}$ M. Urdampilleta, S. Klyatskaya, J. Cleuziou, M. Ruben, and W. Wernsdorfer, "Supramolecular spin valves," Nat. Mater. 10, 502-506 (2011).

${ }^{252}$ S. Voss, M. Fonin, L. Burova, M. Burgert, Y. S. Dedkov, A. B. Preobrajenski, E. Goering, U. Groth, A. R. Kaul, and U. Ruediger, "Investigation of the stability of $\mathrm{Mn}_{12}$ single molecule magnets," Appl. Phys. A 94, 491 (2009).

${ }^{253}$ B. W. Heinrich, L. Braun, J. I. Pascual, and K. J. Franke, "Protection of excited spin states by a superconducting energy gap," Nat. Phys. 9, 765-768 (2013).

${ }^{254}$ N. Roch, S. Florens, T. A. Costi, W. Wernsdorfer, and F. Balestro, "Observation of the underscreened Kondo effect in a molecular transistor," Phys. Rev. Lett. 103, 197202 (2009).

${ }^{255}$ T. Esat, B. Lechtenberg, T. Deilmann, C. Wagner, P. Krüger, R. Temirov, M. Rohlfing, F. B. Anders, and F. S. Tautz, "A chemically driven quantum phase transition in a two-molecule Kondo system," Nat. Phys. 12, 867 (2016).
${ }^{256}$ S. Sasaki, S. De Franceschi, J. Elzerman, W. van der Wiel, M. Eto, S. Tarucha, and L. Kouwenhoven, "Kondo effect in an integer-spin quantum dot," Nature 405, 764-767 (2000).

${ }^{257}$ The dashed green line in (a) can also be included by allowing a slight breaking of spin-conservation, enabling the $S \leftrightarrow Q$ transition. This more detailed modeling goes beyond the level of detail required for the COSET and COT features on which we focus.

${ }^{258}$ Since this enhancement of the excited $D_{1}$ state is small compared to that of the other "equilibrium" excitations it is not revealed by Fig. 11(b).

${ }^{259}$ Note that this is not a single $\Gamma^{4}$ process, since the two $\Gamma^{2}$ steps occur in sequence. This is similar to the COSET which is not a $\Gamma^{3}$ process, but a sequence of two processes, one of the order of $\Gamma^{2}$ and the other of $\Gamma$.

${ }^{260}$ The downshifting partner excitation is visible at a different gate voltage in Fig. 13(b) (labeled as COSET) and in the lower panel of Fig. 14(a).

${ }^{261}$ In a renormalization group (RG) treatment of the master equation, the broadening arises naturally. First, in uniform way as the sum of all tunnel rates in the discrete RG approximation. Then during a continuous RG flow the broadening is corrected in an energy dependent way.

${ }^{262}$ The nonperturbative hierarchical density-operator approach (HQME) can also deal with the broadening, as demonstrated both for SET and COSET resonances in the cited reference.

${ }^{263}$ C. Schinabeck, A. Erpenbeck, R. Härtle, and M. Thoss, "Hierarchical quantum master equation approach to electronic-vibrational coupling in nonequilibrium transport through nanosystems," Phys. Rev. B 94, 201407(R) (2016).

${ }^{264}$ N. M. Gergs, C. B. M. Hörig, M. R. Wegewijs, and D. Schuricht, "Charge fluctuations in nonlinear heat transport," Phys. Rev. B 91, 201107 (2015).

${ }^{265}$ G. E. W. Bauer, E. Saitoh, and B. J. van Wees, "Spin caloritronics," Nat. Mater. 11, 391-399 (2012). 